\title{
T helper cells modulate intestinal stem cell renewal and
} differentiation

Moshe Biton ${ }^{1,2 *}$, Adam L. Haber ${ }^{1 *}$, Semir Beyaz $z^{3,4}$, Noga Rogel $^{1}$, Christopher Smillie $^{1}$, Karthik Shekhar ${ }^{1}$, Alexandra Schnell ${ }^{5}$, Zuojia Chen ${ }^{5}$, Chuan Wu ${ }^{5}$, Jose Ordovas-Montanes ${ }^{6,7,8}$, David Alvarez ${ }^{9}$, Rebecca H. Herbst ${ }^{1,10}$, Itay Tirosh ${ }^{1}$, Grace Burgin ${ }^{1}$, Danielle Dionne ${ }^{1}$, Michael E. Xifaras ${ }^{3}$, Mei Zhang ${ }^{11}$, Alex K. Shalek $^{6,7,8}$, Ulrich H. von Andrian ${ }^{9}$, Daniel B. Graham ${ }^{7}$, Orit Rozenblatt-Rosen ${ }^{1}$, Hai Ning Shi ${ }^{11}$, Vijay Kuchroo ${ }^{5,7}$, Omer Yilmaz ${ }^{3,7,12}$, Aviv Regev ${ }^{1,3,13} \S$ and Ramnik J. Xavier ${ }^{2,7,14} \S$.

${ }^{1}$ Klarman Cell Observatory, Broad Institute of Harvard and MIT, Cambridge, MA 02142, USA

${ }^{2}$ Center for Computational and Integrative Biology, Massachusetts General Hospital, Boston, MA, 02114, USA

${ }^{3}$ The David H. Koch Institute for Integrative Cancer Research at MIT, Department of Biology, MIT, Cambridge, Massachusetts 02139, USA.

${ }^{4}$ Division of Hematology/Oncology, Boston Children's Hospital and Department of Pediatric Oncology, Dana-Farber Cancer Institute, Howard Hughes Medical Institute, Harvard Stem Cell Institute, Harvard Medical School, Boston, Massachusetts 02115, USA.

${ }^{5}$ Evergrande Center for Immunologic Diseases, Brigham and Women's Hospital, Harvard Medical School, Boston, Massachusetts, USA.

${ }^{6}$ Institute for Medical Engineering Science \& Department of Chemistry, Massachusetts Institute of Technology (MIT), Cambridge, MA 02139, USA.

${ }^{7}$ Broad Institute of Harvard and MIT, Cambridge, MA 02142, USA

${ }^{8}$ Ragon Institute of MGH, MIT, and Harvard, Cambridge, MA 02139, USA

${ }^{9}$ Department of Microbiology \& Immunobiology and Center for Immune Imaging, Harvard Medical School, Boston, MA 02115, USA.

${ }_{11}^{10}$ Department of Systems Biology, Harvard Medical School, Boston, MA 02114, USA

${ }^{11}$ Mucosal Immunology and Biology Research Center, Massachusetts General Hospital and Harvard Medical School, Charlestown, MA, 02129, USA.

${ }^{12}$ Departments of Pathology, Gastroenterology, and Surgery, Massachusetts General Hospital and Harvard Medical School, Boston, Massachusetts 02114, USA

${ }^{13}$ Howard Hughes Medical Institute, Department of Biology, Massachusetts Institute of Technology, Cambridge, MA 02140, USA

${ }^{14}$ Gastrointestinal Unit and Center for the Study of Inflammatory Bowel Disease, Massachusetts General Hospital, Boston, MA, 02114, USA

* These authors contributed equally to this work.

$\S$ These are the equal, co-senior authors. Correspondence should be addressed to RJX (xavier@molbio.mgh.harvard.edu) or AR (aregev@broadinstitute.org) 


\section{Abstract}

In the small intestine, a cellular niche of diverse accessory cell types supports the rapid generation of mature epithelial cell types through self-renewal, proliferation, and differentiation of intestinal stem cells (ISCs). However, not much is known about interactions between immune cells and ISCs, and it is unclear if and how immune cell dynamics affect eventual ISC fate or the balance between selfrenewal and differentiation. Here, we used single-cell RNA-seq (scRNA-Seq) of intestinal epithelial cells (IECs) to identify new mechanisms for ISC-immune cell interactions. Surprisingly, $\mathrm{MHC}$ class $\mathrm{II}(\mathrm{MHClI})$ is enriched in two distinct subsets of $\mathrm{Lgr5}^{+}$crypt base columnar ISCs, which are also distinguished by higher proliferation rates. Using co-culture of $\mathrm{T}$ cells with intestinal organoids, cytokine stimulations, and in vivo mouse models, we confirm that $\mathrm{CD}^{+} \mathrm{T}$ helper (Th) cells communicate with ISCs and affect their differentiation, in a manner specific to the Th subtypes and their signature cytokines and dependent on MHCII expression by ISCs. Specific inducible knockout of MHCII in intestinal epithelial cells in mice in vivo results in expansion of the ISC pool. Mice lacking T cells have expanded ISC pools, whereas specific depletion of $\mathrm{T}_{\text {reg }}$ cells in vivo results in substantial reduction of ISC numbers. Our findings show that interactions between Th cells and ISCs mediated via MHCII expressed in intestinal epithelial stem cells help orchestrate tissue-wide responses to external signals. 


\section{Introduction}

The intestinal mucosa maintains a functional equilibrium with the complex luminal milieu, which is dominated by a spectrum of gut microbial species and their products. The functional balance between the epithelium and the lumen plays a central role in maintaining the normal mucosa and in the pathophysiology of many gastrointestinal disorders [1]. To maintain barrier integrity and tissue homeostasis in response to immune signals and luminal contents [1], the gut epithelium constantly regenerates by rapid proliferation and differentiation [2]. This process is initiated by intestinal stem cells (ISCs), which give rise to committed progenitors that in turn differentiate to specific IEC types [3, 4].

ISC differentiation depends on external signals from an ecosystem of nonepithelial cells in the gut niche. In particular, canonical signal transduction pathways, such as Wnt and Notch [5, 6], are essential to ISC maintenance and differentiation, and rely on signals from stromal cells $[7,8]$. The intestinal tract is also densely populated by innate and adaptive immune cells, which maintain the balance between immune activation and tolerance $[1,9]$. However, it is unknown if and how immune cells and the adjacent ISCs interact.

Several studies suggest an important role for immune cells in tissue homeostasis. Tissue-resident innate immune cells, such as macrophages and type 3 innate lymphoid cells (ILC3s), can play a role in regeneration of the gut [7, 10] and other tissues $[11,12]$. Among adaptive immune cells, recent studies 
have implicated $T$ regulatory cells $\left(T_{\text {regs }}\right)$ in regeneration within muscles, lungs, and the central nervous system [13-15]. Skin-resident $\mathrm{T}_{\text {regs }}$ were very recently shown to be involved in maintaining hair follicle stem cell (HFSC) renewal through Jagged1-mediated Notch signaling [16]. In the gut, mouse models of intestinal infection, $\mathrm{T}$ cell depletion, and inflammatory bowel disease (IBD) all display aberrant epithelial cell composition, such as goblet cell hypoplasia or tuft cell expansion [17-20]. These phenotypes have been primarily interpreted as reflecting intestinal epithelial cell dysfunction and changes in gut microbial populations $[9,17,21,22]$.

Here, we set out to identify and characterize novel mechanisms for interaction between immune cells and ISCs. Using scRNA-seq, we identified a putative molecular mechanism for $\mathrm{CD}^{+} \mathrm{T}$ cell interaction with specific subsets of $\mathrm{Lgr}^{+}$ ISCs with enriched expression of $\mathrm{MHC}$ class II (MHCII) molecules and higher proliferation rates. We characterized this putative interaction using SCRNA-Seq and in situ analysis of canonical in vivo infection models, organoid assays, and T

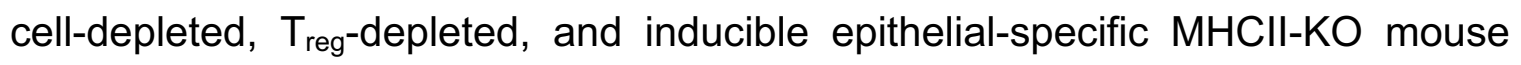
models. We found that $\mathrm{CD}^{+} \mathrm{T}$ helper cells influence ISC renewal and epithelial differentiation via $\mathrm{MHCll}$ interaction. Our study underscores the important anatomic positioning of $\mathrm{CD}^{+} \mathrm{T}$ cell-ISC interactions in the context of ISC renewal or contraction, gut inflammation, and tumorigenesis.

\section{Results}




\section{High expression of MHCII genes by ISC subsets}

To identify potential mechanisms for ISC-immune cell interactions, we searched for genes that are specifically expressed by ISCs compared to other gut epithelial cells and that encode cell surface or secreted proteins capable of interacting with cognate molecules on immune cells. We collected full-length, high-coverage scRNA-seq (scRNA-seq) data of 1,522 $\mathrm{EpCAM}^{+}$intestinal epithelial cells (IECs) [18] from crypt-enriched small intestine of WT and Lgr5-GFP mouse models [23] (Methods). Using unsupervised clustering ( $k$-nearest neighbor ( $k$-NN) graphbased clustering, Methods) of the 1,522 cells (Table S1) we identified 637 Lgr5high ( Lgr5 $^{\text {High }}$ ) stem cells (Figure S1A,B), as well as clusters corresponding to mature enterocytes, Paneth, goblet, tuft, and enteroendocrine cells [18]. Clustering of only the 637 ISCs (Methods) further partitioned the ISCs into three distinct subsets (ISC-I, -II and -III, Figure 1A,B), all of which express known stem cell markers [3] including Lgr5 (Figure S1C). This was consistent with recent scRNA-seq reports $[24,25]$. We confirmed that all three subsets comprise $\operatorname{Lgr}^{+}$ ISCs using the Lgr5-GFP mouse model [26]: the three stem cell populations were strongly enriched for GFP ${ }^{\text {high }}$ cells (Figure S1D), over $90 \%$ of the GFP ${ }^{\text {high }}$ cells were allocated to one of the three stem cell subsets (Figure S1E), and the three subsets are present in similar proportions in the duodenum, jejunum, and ileum (Figure S1F and Methods). Lastly, we identified differentially expressed genes between the three subsets, as well as between all ISCs and the other IECs that are annotated as receptors or ligands for cell-cell interactions [27] (Figure S1G and Table S2). 
We found that $C D 74$, the invariant chain of the MHCIl complex, was highly expressed and enriched in ISCs (Figure 1C-E and Figure S1G). Moreover, other MHCII genes were among the most strongly expressed by two out of three ISC subsets (ISC-II and -III) (Figure 1C,D) compared to other IECs (Figure S2A). These included many canonical components of the MHCll machinery, including $H 2-A b 1, H 2-D M b 1, H 2-D M a, H 2-A a, C d 74$, and the recently discovered costimulatory molecules Sectm1a and Sectm1b [28] (Figure 1D), but not the canonical co-stimulatory molecules CD80 and CD86 (data not shown). Although MHCII expression has been previously reported in intestinal epithelial cells [2932], it was not shown to be enriched in ISCs. We found that MHCll expression in the ISC $^{\mathrm{MHCll+}}$ (ISC-II and -III) groups was the highest among all IECs at both the mRNA and protein levels (Figure S2A,B). We confirmed MHCll protein expression by ISCs using an immunofluorescence assay (IFA) and immunohistochemistry (IHC) with anti-MHCIl antibodies in wild type mice, and its absence in a constitutive MHCII knockout (KO) [33] (Figure 1E and Figure S2C).

\section{MHCII-expressing ISCs are more proliferative}

The three ISC subsets vary not only in their expression of the MHCll system, but also in their expression of signatures of the cell-cycle [34, 35] (Figure 2A,B). The subset with highest MHCIl expression consisted primarily of cells in G1/S. The second subset, with lower but significant MHCIl expression, had cells spanning several phases of the cell-cycle including G2/M. We concluded that cells in both 
of these subsets are likely in highly proliferative states and termed these ISC-II and ISC-III, respectively. In contrast, the cells in the subset with low or no detectable expression of MHCII, termed ISC-I, also had low G1/S and G2/M scores (129 of 209 cells) and likely represented cells in G0. The low-cycling state of ISC-I was further supported by the higher expression of the histone methylase $K d m 5 b$, which is highly expressed in post-mitotic differentiated cells of the small intestine (Figure S2D,E) and in low-cycling or quiescent cells in other systems $[34,36-39]$. Such heterogeneity in the proliferative state of ISCs has been recently reported, including a quiescent ISC subset, which is enriched for Mex3a and correlates well with our low cycling ISC-I subset (data not shown) [24, 25]. Importantly, while the cell-cycle status aligns with the partitioning of ISC-I, II and III subsets, the ISC subsets are discernable even when we exclude canonical cell-cycle genes (Figure 2C, Figure S3A, Table S3 and Methods) and even when analyzing only the 183 ISCs scoring at G0 (Figure S3B).

We validated the association between the ISC subsets, MHC-II, and cell-cycle status by co-staining in situ (Figure 2D), by in vivo EdU labeling followed by single-nucleus RNA-seq (Div-Seq) [40] (Figure 2E), and by determining the proportion of $\mathrm{EdU}^{+}$cells in subsets of GFP ${ }^{\text {high }}$ cells with different levels of MHCII expression (Figure 2F). We also sorted single $\mathrm{MHCII}^{\text {high }}$ and $\mathrm{MHCII}{ }^{\text {low }}$ ISCs from Lgr5-GFP mice and collected 503 full-length scRNA-seq profiles. MHCII ${ }^{\text {high }}$ ISCs had a higher proportion of cells with high scores for ISC-II and -III state signatures, whereas $\mathrm{MHCII}^{\text {low }}$ ISCs had a higher proportion of cells scoring highly 
for the ISC-I state, consistent with our in silico analysis (Figure 2G and Figure S3C). Taken together, these results support an association between $\mathrm{MHCII}$ expression and proliferative state within ISCs in vivo. Thus, the heterogeneity of $\mathrm{Lgr}^{+}$stem cells and MHC II expression is correlated with proliferation rates.

\section{T helper subsets and their signature cytokines regulate ISC renewal in organoids}

We hypothesized that ISCs may interact with $\mathrm{CD}^{+} \mathrm{T}$ helper (Th) cells via $\mathrm{MHCII}$ recognition and, as a consequence, $\mathrm{CD}^{+}$Th cells may affect ISC fate via cytokine-receptor interaction. Importantly, IECs, including ISCs, express receptors for Th cytokines interferon gamma (IFN $\gamma$ ), interleukin-10 (IL-10), IL-13, IL-4 and IL-17A (data not shown). Furthermore, intra-vital imaging showed that $\mathrm{CD}^{+}$Th cells can be in very close proximity to stem cells in small intestinal crypts (Figure S4A and movie S1). Moreover, scRNA-seq of IECs following infection of mice with Salmonella enterica (Salmonella) or Heligmosomoides polygyrus (H. polygyrus) [18], which induce Th1 (Figure S4B-D and Table S4) and Th2 responses, respectively, shows not only distinct shifts in the proportions of post-mitotic cells, such as tuft (in H. polygyrus) and Paneth (in Salmonella) cells [18] (Figure S4E-F), but also a reduction in ISC and stemness programs, and especially in ISC-I cells (Figure S4G-J). The observed elevation in ISC ${ }^{\mathrm{MHCII+}}$ programs is consistent with our hypothesis, but in principle many other indirect 
cellular and molecular mechanisms in the complex cellular ecosystem of the crypt may also be involved.

To dissect the potential interactions between $\mathrm{T}$ helper cells and ISCs independently of other contributions to the niche, we therefore next used the intestinal organoid system [26] in which immune cells are natively absent but can be added in a controlled manner [41]. We introduced either specific CD4 ${ }^{+} \mathrm{T}^{-}$ helper subsets (Figure S5A) or their corresponding signature cytokines to organoid cultures, and used scRNA-seq to identify changes in the proportions or expression programs of ISCs. In one set of experiments, we co-cultured organoids with $\mathrm{CD}^{+} \mathrm{T}$ cells that were polarized ex vivo towards Th1, Th2, Th17, and $\mathrm{iT}_{\text {reg }}$ cells [42] (Figure S5B). In a parallel set, we stimulated organoids derived from C57BL/6J WT mice with key cytokines produced by each of the four T helper subsets: IFNy (Th1), IL-13 (Th2), IL-17a (Th17), and IL-10 (inducible $\mathrm{T}_{\text {reg, }} \mathrm{iT}_{\text {reg }}$ ). In each experiment, we collected droplet-based scRNA-seq profiles (Methods). For co-cultures, we computationally distinguished (post-hoc) T cells from epithelial cells by their profiles (Methods) and confirmed the Th cell state by mRNA expression of signature cytokines and transcription factors (Figure S5C). Although ex vivo polarized $\mathrm{T}$ helper cells share many hallmarks with their in vivo counterparts, they do not perfectly recapitulate them. In particular, Th2 differentiation yielded only $16.5 \%$ IL-4 and IL-13 expressing cells, while other T helper subsets had higher differentiation rates (Figure S5B). There are also several differences between organoids and in vivo IECs (Figure S5D-G): 
Organoids are enriched for stem cells [26, 43] (Figure S5D), the goblet and Paneth lineages do not fully diverge (Figure S5E), also in independentlygenerated organoids [44] (Figure S5F), and MHCIl expression was not detected in our organoid culture (Figure S5G); thus, any impact of Th cell co-cultures is likely mediated through cytokine secretion from the polarized Th cells.

Each of the Th co-cultures or corresponding cytokine treatments resulted in a distinct modulation of the organoid ISC compartment (Figure $3 \mathbf{A}, \mathbf{B}$ and Figure S6A-C). Strikingly, co-cultures with $\mathrm{iT}_{\text {regs }}$ and treatment of organoids with their associated cytokine IL-10 led to organoid ISC expansion (Figure 3A,B, Figure S6A-C, Methods and Table S5), while co-cultures with Th1, Th2 and Th17 cells or treatment with $\mathrm{IL}-13$ or $\mathrm{IL}-17$ all reduced the size of the ISC pool of the organoids. Consistent with their depleted stem cell pool, organoids co-cultured with Th1, Th2, or Th17 cells or treated with IL-17a all showed elevated numbers of TA cells $\left(p<10^{-4}\right.$, hypergeometric test, Figure 3A,B). Note, for IFNy, we used a low concentration $(0.5 \mathrm{u} / \mathrm{ml})$ to avoid organoid apoptosis [45], which did not elicit any effect, while Th1 co-culture resulted in elevation of $\mathrm{MHCll}$ signature in IECs (Figure 3A, top and Figure S5G). In addition, the treatments impacted cell differentiation: IL-13 treatment decreased the proportion of secretory 'Panethgoblet' cells, and increased tuft cells (Figure 3A,C) [19, 46]; Th1 co-culture upregulated Paneth cell-specific genes (Figure S6D-F), consistent with in vivo observations (Figure S4F); and Th2 cell co-cultures had the opposite effect (Figure S6D,E). 
The effects of Th cell subsets and cytokines on ISC numbers suggest that they affect ISC renewal potential, which in turn should affect the ability of ISCs to form organoid cultures. To test this hypothesis, we assessed whether key cytokines affect ISC clonogenicity [47]. We reseeded equal numbers of cytokine-treated organoids in new cultures and quantified the number of organoids after three days ( $n=6$ replicates per each group, Methods). Consistent with our hypothesis, there was a significant reduction in the clonogenicity of organoids treated with the ISC-reducing cytokine IL-13, whereas the ISC-expanding cytokine IL-10 induced higher clonogenicity (Figure 3D), confirming the ability of this $T_{\text {reg-generated }}$ cytokine to rejuvenate the stem cell pool.

\section{Elevation in ISC pool under epithelial MHCII ablation in vivo}

Since the MHCII system is not expressed in organoids, we next assessed its role in IECs in vivo by its conditional KO. We crossed $\mathrm{H} 2-\mathrm{Ab}^{\mathrm{fl} / \mathrm{fl}}[48]$ to Villin-Cre-ER ${ }^{\mathrm{T} 2}$ [49] mice, generating a mouse model of specific and inducible MHCIl knockout in IECs $\left(\mathrm{MHClI}^{\Delta \text { gut }}\right)$. We profiled 1,559 IECs from the $\mathrm{MHClI}^{\Delta \text { gut }}$ mice $(n=5) 10$ days after Tamoxifen induction and 1,617 IECs from floxed control $(\mathrm{MHCII} / \mathrm{flfl})$ littermates ( $n=5$ mice). We validated that MHCII is successfully knocked-out in EpCAM $^{+}$IECs (Figure 4A and Figure S7A), but not in CD11 ${ }^{+}$dendritic cells in the mesenteric lymph node (Figure S7B). 
Strikingly, the fraction of $\mathrm{Lgr5}^{+}$cells was $31.3 \%$ higher in $\mathrm{MHCII}{ }^{\Delta \text { gut }}$ mice $(p<0.05$, likelihood-ratio test, Figure S8A), which we confirmed by Lgr5-smFISH (Figure 4B), and the proportion of ISCs as defined by unsupervised clustering (Figure S8B,C) which was $17.6 \%$ higher (FDR<0.05, likelihood-ratio test, Figure 4C). Consistently, stem cell markers are overrepresented $\left(p<10^{-6}\right.$, hypergeometric test, Figure S8D) among the genes up-regulated in the $\mathrm{MHCll}^{\Delta \text { gut }}(\mathrm{FDR}<0.05$, likelihood-ratio test), including canonical ISC markers (e.g., Lgr5, Olfm4, Smoc2,

and Igfbp4, Figure S8D). Furthermore, we separately analyzed only $\mathrm{MHCI}^{\Delta \text { gut }}$ ISCs in which $H 2-A b 1$ is confirmed to be silenced (defined as no detectable mRNA) or only $\mathrm{MHCII}^{\Delta \text { gut }}$ ISCs in which $\mathrm{H} 2-A b 1$ mRNA is still expressed (Figure S8E, bottom left vs. right). We find that in $\mathrm{MHCII}^{\Delta \text { gut }}$ ISCs in which $H 2-A b 1$ is confirmed to be silenced, expression of stem cell markers [3] was significantly higher than in stem cells still expressing $H 2-A b 1$ ( $p<0.05$, likelihood-ratio test). Finally, the ISC-III signature score was significantly lower in stem cells from $\mathrm{MHCI}^{\mathrm{\Delta gut}}$ mice (Figure 4D), suggesting that the ISCs in the expanded pool are shifted toward the ISC ${ }^{\mathrm{MHCII}-}$ state. Taken together, these data suggest that $\mathrm{MHCII}^{\Delta \mathrm{gut}}$ increased ISC numbers and the expression of stem cell markers.

\section{T cells modulate ISC renewal and differentiation in vivo}

Our organoid assays predicted that Th cell subsets have distinct effects on intestinal epithelial cell differentiation. To demonstrate the relevance of the $\mathrm{T}$ cellISC interaction in vivo, we first assessed two $\mathrm{T}$ cell-deficient mouse models. 
First, we profiled 2,967 individual IECs isolated from athymic B6 nude mice [50] $(n=2)$, characterized by $\mathrm{T}$ cell depletion. Unsupervised clustering revealed a markedly higher fraction of stem cells $\left(52.5 \%\right.$ increase, $\mathrm{FDR}<10^{-3}$, likelihood-ratio test, Methods) compared to control mice ( $n=6$, Figure S9A,B,E). Consistently, stem cell markers were enriched (56 of 1,804 genes, $p<10^{-6}$, hypergeometric test, Figure S9A and Table S6) among genes overall up-regulated in cells of nude vs. controls (FDR $<0.05$, likelihood-ratio test). Similar analysis of 9,488 individual IECs profiled from TCR $\beta-K O$ mice $(n=2)$ [51], characterized by a lack of $\alpha / \beta$ T cells, also showed a significant expansion of the ISC pool $(35.0 \%$ increase, FDR $<0.05$, likelihood-ratio test; Figure S9C-E). We confirmed the increased ISC numbers in situ in both T cell depleted models using Lgr5 singlemolecule FISH (smFISH, Figure S9F).

\section{$\mathrm{T}_{\text {reg }}$ cells are essential to maintain the ISC niche in vivo}

Our organoid assays also predicted that $\mathrm{T}_{\text {reg }}$ cells promote renewal of the ISC pool. To test for this effect in vivo, we used the Foxp3-DTR mouse [52], in which $T_{\text {reg }}$ cells are specifically depleted upon application of diphtheria toxin (DT). We profiled 3,387 IECs from both Foxp3-DTR $(n=4)$ and matched control mice $(n=5)$ treated with DT for 7 days and confirmed $\mathrm{T}_{\text {reg }}$ ablation in the lamina propria (Figure S10A). At this time point, there were no signs of increased cell death in the small intestinal crypts or of major tissue inflammation in IECs of Foxp3-DTR vs. control mice (Figure S10B), suggesting that the longer term effects of $\mathrm{T}_{\text {reg }}$ 
depletion are not yet apparent [52]. However, consistent with our hypothesis, there was a substantial reduction $(66.3 \%$ decrease, FDR $<0.005$, likelihood-ratio test) in ISC numbers in the epithelia of the Foxp3-DTR mice, as assessed by unsupervised clustering (Figure 4E), the fraction of cells in which Lgr5 mRNA was detected ( $p<0.005$, likelihood-ratio test, Figure S10C), and smFISH (Figure 4F). Consistently, stem cell marker genes were overrepresented among those

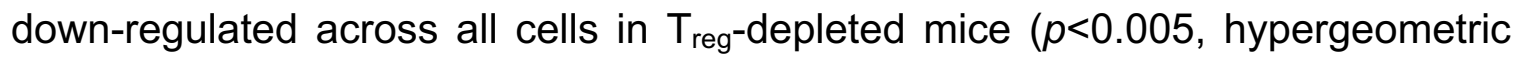
test, Figure S10D). There was also a substantial depletion of mature enterocytes (5.8-fold decrease, FDR $\left.<10^{-5}\right)$, and expansion of tuft cells (4.1-fold increase, FDR<0.005) (Figure S10E), which we confirmed by IFA staining (Figure S10F). We did not observe significant changes in the expression of Notch signaling pathway components $(p=1)$, or Notch targets [53] ( $p=0.31$, hypergeometric test), which a recent study implicated in regulation of hair follicle stem cells by $\mathrm{T}_{\text {regs }}$ [16] (data not shown).

All cell types in the Foxp3-DTR mice, including ISCs, showed strongly elevated expression of MHCII genes ( $p<5 \times 10^{-4}$, likelihood-ratio test, Figure 4G). Amongst stem cells, there was an increase in proliferation, as indicated by both the distribution of cell-cycle signature scores and mKi67 staining $\quad(p<0.005$, likelihood-ratio test, Figure 4F and Figure S10B). Furthermore, and also consistent with our predictions, ISCs from $\mathrm{T}_{\text {reg-ablated Foxp3-DTR mice had an }}$ increased proportion of MHCII positive, proliferative ISCs and a decrease in ISC $^{\mathrm{MHCII-}}$ (ISC-I, Figure 4D). 
bioRxiv preprint doi: https://doi.org/10.1101/217133; this version posted November 14, 2017. The copyright holder for this preprint (which was not certified by peer review) is the author/funder. All rights reserved. No reuse allowed without permission. 


\section{Discussion}

Previous studies of stem cell dynamics and differentiation processes [54, 55], focused on the role of the epithelial-intrinsic or stromal niche signals using lineage tracing. Here, we investigated the possibility of interactions between adaptive immune cells and ISCs. Combining scRNA-seq with homeostatic or perturbed conditions that manipulate either $\mathrm{T}$ helper cells, their cytokines, or MHCII expression by epithelial cells allowed us to assay comprehensive "snapshots" of ISC abundance and the fate of their progeny, followed by in silico inference of cell states and differentiation. In unperturbed mice, the expression of $\mathrm{MHCII}$ is high yet variable across ISCs, such that both ISC-II and III $\left(\mathrm{ISC}^{\mathrm{MHClI+}}\right)$ express high levels of the MHCII molecules, whereas ISC-I (ISC ${ }^{\mathrm{MHCll}-}$ ) do not. Using controlled manipulation experiments in organoids and mice followed by scRNA-seq, we established a crosstalk between Th cells and ISCs.

In particular, our in vitro and in vivo results support a model in which Th cells interact with ISCs via MHCII molecules, impacting the ISC pool and resultant differentiation pathways through their key cytokines (Figure 4I). In this model, $\mathrm{T}_{\text {reg }}$ cells, which are enriched in the small intestine, maintain the ISC niche. They may be elevated after a strong inflammatory response [56] to serve as a feedback effectors in order to replenish and maintain stem cell numbers. Conversely, Th1 and Th2 cells or their signature cytokines both reduce ISC numbers, and bias IEC differentiation toward specific epithelial cell-types, 
perhaps in order to respond to either bacterial (Th1 cells, Paneth cell increase) or parasitic (Th2 cells, tuft cell increase) insults. Th17 cells, which are highly enriched in the small intestine [57], reduce the number of ISCs, which may reflect a shift in the balance between stem cell renewal and differentiation. In this way, epithelial and immune response could be integrated to titrate responses to dense luminal flora, avoiding continuous inflammation, while reacting to pathogens: first, the intestinal stem cells utilize the equilibrium of pro-inflammatory and antiinflammatory signals to balance between renewal and differentiation; second, distinct Th cell subsets can boost the desired immune response by affecting renewal and differentiation processes of the gut epithelia concordantly with signals arriving from the gut lumen. If this novel role for $\mathrm{MHCll}$ in $\mathrm{T}$ cell communication with stem cells also exists in other mucosal or non-mucosal compartments, it may open the possibility of a general mechanism in which adaptive immune cells regulate parenchymal stem cells in order to maintain tissue homeostasis under normal and pathological conditions. 


\section{Figure Legends}

Figure 1. Single-cell RNA-seq reveals MHCII expression in subsets of Lgr5 intestinal stem cells. A,B. Three subsets of intestinal stem cells (ISCs). Shown are a $t$-distributed stochastic neighbor (tSNE) embedding (A) and correlation matrix (B) of 637 intestinal stem cells identified by unsupervised clustering from 1,522 full-length scRNA-seq profiles (Figure S1A, Methods). Individual points in the tSNE embedding (A) correspond to single cells colored by their assignment based on kNN-graph-clustering (Methods) and post-hoc annotation (legend, top left). Heatmap (B) shows the Pearson correlation coefficient ( $r$, color bar) between scores of individual cells (rows and columns) along the first 10 principal components (PCs). Color code marks ISC subsets, (bottom, Methods) C. MHCII expression in Lgr5 ${ }^{+}$ISCs. Violin plot of the distribution of the mean expression levels $\left(\log _{2}(\mathrm{TPM}+1), y\right.$-axis) of MHCII genes $(H 2-A b 1, H 2-A a, C i i t a, C d 74, H 2-$ $D M a, H 2-D M b 1)$ in each of the three ISC groups. D. Antigen presentation genes are enriched in $\mathrm{Lgr5}^{+}$ISCs. Heatmap shows the relative mean expression (rowwise Z-score of $\log _{2}(\mathrm{TPM}+1)$ values, color bar) of $\mathrm{MHCll}$-related genes (rows) in each of the IEC types (columns) as defined by clustering of the 1,522 full-length scRNA-seq profiles. EP: Enterocyte progenitor, EEC: enteroendocrine cell. E. Validation of MHCII expression by ISCs. IFA of MHCII (I-A/I-E; green) co-stained with Paneth cell marker, Lyz1 (red) within the intestinal crypt of a wild type (WT, top row) and $\mathrm{MHCll}$ constitutive knockout ( $\mathrm{MHCll} \mathrm{KO}$, bottom row) mouse. Yellow arrows: MHCII-expressing ISCs adjacent to $\mathrm{Lyz} 1^{+}$Paneth cells at the bottom of the crypt. Scale bar, $20 \mu \mathrm{m}$. 


\title{
Figure 2. MHCll expression is correlated with ISC proliferation.
}

\begin{abstract}
A,B. Distinct cell-cycle characteristics in the three ISC subsets. (A) Violin plot
\end{abstract} shows the distribution of expression scores ( $y$-axis) for a signature of cell-cycle genes (Methods) in each stem cell subset $\left(x\right.$-axis). ${ }^{* *} p<10^{-5},{ }^{* * *} p<10^{-7}$ (MannWhitney U-test). (B) Scatter plots show the signature score for 637 ISCs (points, Methods) for G1/S genes ( $x$-axis) and G2/M genes ( $y$-axis). Cells are colored by their cluster assignment to ISC subsets. C. Gene signatures of ISC subsets. Heatmap shows relative expression level (row-wise Z-score of $\log _{2}(T P M+1)$ values, color bar) of ten representative genes from each ISC subset signature and a pan-stem signature (rows, right color bar) in 637 individual stem cells and 201 TA progenitors (columns, bottom color bar as in (A)) identified from 1,522 scRNA-seq profiles. Gene signatures are identified based on our analysis as well as from a previously published signature of stem cell genes of a bulk dataset [3]. D. Identification of proliferating stem cells in intestinal crypts. Upper panels: Combined single-molecule fluorescence in situ hybridization (smFISH) with immunofluorescence (IFA) of FFPE sections of intestinal tissue from wild type mice, showing the pan-stem cell marker Lgr5 (upper left), ISC-I marker Cyp2e1 (upper middle) and ISC-III marker Psrc1 (upper right) all in red and mKi67 in

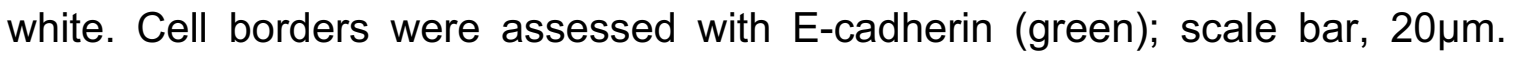
Bottom left: Schematic of the lower crypt fraction ('stem cell zone'), in which coexpression of stem cell markers (Lgr5 and Cyp2e1 or Psrc1) and the proliferation 
marker mKi67 was quantified. Bottom right: Bar plot showing the fraction ( $y$-axis) of cells which are positive for mKi67 out of all cells positive for each stem cell marker (lower right panel). $n=4$ mice, 10 crypts on average per mouse (** $p<0.0025, t$-test; error bars: SEM). E. Enrichment of ISC-I in EdU' cells. Violin plots show the distribution of signature scores for the cell-cycle (left, signature as in (A)) and ISC-I (right, signature as in (C)) from FACS sorted EdU Lgr5 $^{+}$ISCs (light green) or $\mathrm{EdU}^{+} \mathrm{Lgr5}^{+}$ISCs (dark green) after in vivo EdU labeling and profiled using single-nucleus RNA-seq (Div-Seq). F. Higher proliferation of ISCs with high MHCII expression. FACS plots of ISCs gated on GFP-high ( $\mathrm{Lgr5}^{+}$, left) binned into subsets with low, intermediate and high $\mathrm{MHCll}$ expression (middle panels, $y$-axis), and then gated on EdU incorporation (middle panels, $x$-axis). Bar plot (right) shows the fraction (percentage, $y$-axis) of $\mathrm{EdU}^{+}$cells within each MHCII expression level $\left(n=4\right.$ mice, ${ }^{*} p<0.05,{ }^{* *} p<0.005,{ }^{* * *} p<0.0005, t$-test, error bars: SEM). G. ISC subset signatures across MHCIl expression. Violin plots show the distribution of signature scores (as in (C)) for ISC-I, ISC-II, and ISC-III subsets (left to right), across scRNA-seq profiles from $326 \mathrm{Lgr}^{+} \mathrm{MHCll}^{\text {high }}$ (light blue) and $177 \mathrm{Lgr}^{+} \mathrm{MHCI}^{\text {low }}$ (dark blue) cells (individual black dots). Horizontal black line denotes the median $\left({ }^{* *} p<0.005,{ }^{* * *} p<0.0005\right.$, Mann-Whitney U-test). 
Figure 3. T helper cells and their key cytokines impact ISC number and differentiation in intestinal organoids. A,B. Shifts in cell type composition within organoids treated with $\mathrm{CD}^{+}$Th signature cytokines $(\mathbf{A})$ or co-cultured with Th cells (B). relative abundance ( $y$-axis) of each IEC-type under each condition relative to their proportions in control organoids (dashed line). Subsets were identified by unsupervised clustering of 23,177 single cells obtained from the Th co-culture and cytokine conditions and annotated post-hoc (Figure S5D, Methods). ${ }^{*} p<0.01,{ }^{* *} p<10^{-4}$ (hypergeometric test, Methods) C. DCLK1 ${ }^{+}$tuft cell expansion following IL-13 treatment. IFA of DCLK1 (red) in control organoids

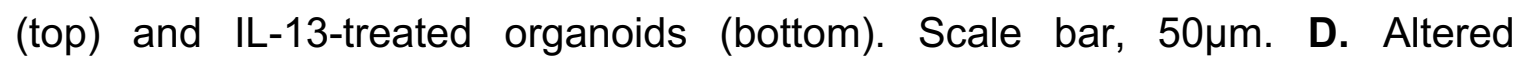
clonogenicity following pre-treatment with different cytokines. Bar plot shows the relative clonogenicity of organoid cultures ( $y$-axis, relative to the mean value of control organoids) defined by the number of organoids in cultures re-seeded after treatment with IL-10 or IL-13 ( $x$-axis). Dots: technical replicates. Error bars: SD, * $p<0.05,{ }^{* * *} p<0.0005, t$-test. 
Figure 4. MHCIl depletion leads to increased ISC numbers while $T_{\text {reg }}$ ablation results in reduced ISC pool. A-D. Expansion of the ISC pool following epithelial specific ablation of MHCII. (A) Validation of epithelial-specific MHCII$\mathrm{KO}\left(\mathrm{MHCII}{ }^{\Delta \text { gut }}\right)$ mouse. IFA of Lyz1 (red) and MHCII (I-A/I-E, green) in MHCII fl/fl (top row) and $\mathrm{MHCI}^{\Delta \text { gut }}$ mice (bottom row). Yellow arrow: $\mathrm{MHCl}^{+}$epithelial cell, white arrow: $\mathrm{MHClI}^{+}$non-epithelial cell. (B) smFISH of the expression of Lgr5 (red) within intestinal crypts from $\mathrm{MHCI}^{\mathrm{fl} / \mathrm{fl}}$ controls $\left(n=5\right.$, top left) and $\mathrm{MHCI}^{\Delta \text { gut }}$ mice ( $n=5$, bottom left). Bar plot (right) shows the number of Lgr5 mRNA molecules per crypt (y-axis) in $\mathrm{MHCII}^{\mathrm{fl} / \mathrm{fl}}$ and $\mathrm{MHCII}^{\Delta \text { gut }}$ mice ( $x$-axis). $n=2$ mice and 8 fields per group. Error bars: SD ( ${ }^{*} p<0.05, t$-test). (C) Bar plot shows the fraction of ISCs within $\mathrm{EpCAM}^{+}$cells ( $y$-axis, determined by unsupervised clustering) in $\mathrm{MHCII}^{\mathrm{flfl} f}$ and $\mathrm{MHCII}^{\Delta \text { gut }}$ mice (points, $x$-axis). Error bars are SEM. (* FDR $<0.05$, likelihood-ratio test, Methods). (D) Heatmap shows the significance of changes in signature scores $\left(-\log _{10}(p\right.$-value), Mann-Whitney U-test, of enrichment (red) and depletion (blue), Methods) of $\mathrm{MHCII}^{\Delta \text { gut }}$ or $\mathrm{T}_{\text {reg }}$ ablation compared to control mice (columns) for signatures (rows) associated with the three ISC subsets. E-H. Reduction in ISC numbers and shifts toward ISC-II and ISC-III states in $\mathrm{T}_{\text {reg }}$ depleted mouse model. (E) Bar plot shows the fraction of ISCs within EpCAM ${ }^{+}$cells ( $y$-axis) detected by unsupervised clustering in WT and Foxp3-DTR mice both treated with DT). Error bars are SEM. ( ${ }^{* *}$ FDR $<0.005$, likelihood-ratio test, Methods). (F) smFISH of FFPE sections of intestinal tissue from wild type mice (top) or Foxp3-DTR mice (bottom) both treated with DT, showing from left to right, the stem cell marker Lgr5 (red), mKi67 (white) and a 
merge; scale bar, $20 \mu \mathrm{m}$. Bar plot (right) shows the number of Lgr5 mRNA molecules per crypt (y-axis) in WT and Foxp3-DTR mice treated with DT ( $x$-axis). $n=2$ mice and 8 fields per group, error bars are SD $\left({ }^{* * *} p<0.001, t\right.$-test $) .(\mathbf{G})$ Immunohistochemistry (IHC) of MHCII (I-A/I-E; brown) co-stained with hematoxylin (blue) within the intestinal crypt of wild type (top) and Foxp3-DTR (bottom) mice both treated with DT. Red arrows indicate $\mathrm{MHCI}^{+}$ISCs. (H) A shift towards proliferative ISC states following $\mathrm{T}_{\text {reg }}$ depletion. Violin plots of the distribution of scores (Methods) for the cell-cycle (left; as in Figure 2) and MHCII genes (right; as in Figure 1) in ISCs (small points) from WT ( $n=5 ; 2,572$ cells) and Foxp3-DTR ( $n=4,815$ cells) mice. Squares: mean score per mouse; thick bar: overall mean; error bars: SEM. ( ${ }^{*} p<0.05,{ }^{* *} p<0.005,{ }^{* * *} p<5 \times 10^{-4}$, likelihood-ratio test). I. Proposed model of a novel interaction between $\mathrm{CD}^{+}{ }^{+}$ helper cells and ISCs mediated by MHCII. T helper cell subsets (blue nodes) modulate (solid arrows) the differentiation (dashed arrows) of ISCs (green). $\mathrm{T}_{\text {regs }}$ and their key cytokine IL-10 promote stem cell renewal, while Th17 cells and their cytokine IL-17a reduce stem cell renewal and promote differentiation. Both Th1 and Th2 suppress stem cell renewal and promote specific differentiation towards Paneth cells (tan) and tuft cells (orange), respectively. 


\section{Acknowledgements}

We thank Leslie Gaffney for help with figure preparation, the Broad Flow Cytometry Facility: Patricia Rogers, Stephanie Saldi and Chelsea Otis and Tim Tickle for help with the Single Cell Portal. Work was supported by the Klarman Cell Observatory at the Broad Institute (AR and RJX), HHMI (AR), and a Broadnext10 award (AR an RJX). MB was supported by a postdoctoral fellowship from the Human Frontiers Science Program (HFSP). RJX funded by DK043351, DK097485 and Helmsley Charitable trust. ASK in a member of Searle Scholars Program, the Beckman Young Investigator Program and the recipient of the NIH New Innovator Award DP2 OD020839. ÖHY is funded by CA211184 and AG045144 NIH grants and is the recipient of the Sidney Kimmel Scholar Award, the Pew-Stewart Trust Scholar Award and a member in the American Federation of Aging Research. AR is a member of the SAB for ThermoFisher Scientific, Syros Pharmaceuticals, and Driver Group. 


\section{References}

1. Peterson, L.W., and Artis, D. (2014). Intestinal epithelial cells: regulators of barrier function and immune homeostasis. Nature reviews. Immunology 14, 141-153.

2. van der Flier, L.G., and Clevers, H. (2009). Stem cells, self-renewal, and differentiation in the intestinal epithelium. Annu Rev Physiol 71, 241-260.

3. Muñoz, J., Stange, D.E., Schepers, A.G., van de Wetering, M., Koo, B.-K., Itzkovitz, S., Volckmann, R., Kung, K.S., Koster, J., Radulescu, S., et al. (2012). The Lgr5 intestinal stem cell signature: robust expression of proposed quiescent \&apos;+4\&apos; cell markers. The EMBO Journal 31, 3079-3091.

4. Tetteh, P.W., Basak, O., Farin, H.F., Wiebrands, K., Kretzschmar, K., Begthel, H., van den Born, M., Korving, J., de Sauvage, F., van Es, J.H., et al. (2016). Replacement of Lost Lgr5-Positive Stem Cells through Plasticity of Their Enterocyte-Lineage Daughters. Cell Stem Cell 18, 203-213.

5. Noah, T.K., Donahue, B., and Shroyer, N.F. (2011). Intestinal development and differentiation. Exp Cell Res 317, 2702-2710.

6. van Es, J.H., van Gijn, M.E., Riccio, O., van den Born, M., Vooijs, M., Begthel, H., Cozijnsen, M., Robine, S., Winton, D.J., Radtke, F., et al. (2005). Notch/gammasecretase inhibition turns proliferative cells in intestinal crypts and adenomas into goblet cells. Nature 435, 959-963.

7. Saha, S., Aranda, E., Hayakawa, Y., Bhanja, P., Atay, S., Brodin, N.P., Li, J., Asfaha, S., Liu, L., Tailor, Y., et al. (2016). Macrophage-derived extracellular vesicle-packaged WNTs rescue intestinal stem cells and enhance survival after radiation injury. Nat Commun 7, 13096.

8. Lei, N.Y., Jabaji, Z., Wang, J., Joshi, V.S., Brinkley, G.J., Khalil, H., Wang, F., Jaroszewicz, A., Pellegrini, M., Li, L., et al. (2014). Intestinal subepithelial myofibroblasts support the growth of intestinal epithelial stem cells. PLoS One 9, e84651.

9. Shale, M., Schiering, C., and Powrie, F. (2013). CD4(+) T-cell subsets in intestinal inflammation. Immunol Rev 252, 164-182.

10. Lindemans, C.A., Calafiore, M., Mertelsmann, A.M., O'Connor, M.H., Dudakov, J.A., Jenq, R.R., Velardi, E., Young, L.F., Smith, O.M., Lawrence, G., et al. (2015). Interleukin-22 promotes intestinal-stem-cell-mediated epithelial regeneration. Nature 528, 560-564.

11. Stappenbeck, T.S., and Miyoshi, H. (2009). The role of stromal stem cells in tissue regeneration and wound repair. Science 324, 1666-1669.

12. Aurora, A.B., and Olson, E.N. (2014). Immune modulation of stem cells and regeneration. Cell Stem Cell 15, 14-25.

13. Dombrowski, Y., O'Hagan, T., Dittmer, M., Penalva, R., Mayoral, S.R., Bankhead, P., Fleville, S., Eleftheriadis, G., Zhao, C., Naughton, M., et al. (2017). Regulatory $\mathrm{T}$ cells promote myelin regeneration in the central nervous system. Nat Neurosci 20, 674-680.

14. Burzyn, D., Kuswanto, W., Kolodin, D., Shadrach, J.L., Cerletti, M., Jang, Y., Sefik, E., Tan, T.G., Wagers, A.J., Benoist, C., et al. (2013). A special population of regulatory T cells potentiates muscle repair. Cell 155, 1282-1295. 
15. Arpaia, N., Green, J.A., Moltedo, B., Arvey, A., Hemmers, S., Yuan, S., Treuting, P.M., and Rudensky, A.Y. (2015). A Distinct Function of Regulatory T Cells in Tissue Protection. Cell 162, 1078-1089.

16. Ali, N., Zirak, B., Rodriguez, R.S., Pauli, M.L., Truong, H.A., Lai, K., Ahn, R., Corbin, K., Lowe, M.M., Scharschmidt, T.C., et al. (2017). Regulatory T Cells in Skin Facilitate Epithelial Stem Cell Differentiation. Cell 169, 1119-1129 e1111.

17. Biton, M., Levin, A., Slyper, M., Alkalay, I., Horwitz, E., Mor, H., Kredo-Russo, S., Avnit-Sagi, T., Cojocaru, G., Zreik, F., et al. (2011). Epithelial microRNAs regulate gut mucosal immunity via epithelium-T cell crosstalk. Nat Immunol 12, 239-246.

18. Haber, A.L., Biton, M., Rogel, N., Herbst, R.H., Shekhar, K., Smillie, C., Burgin, G., Delorey, T.M., Howitt, M.R., Katz, Y., et al. (2017). A single-cell survey of the small intestinal epithelium. Nature doi:10.1038/nature24489.

19. Howitt, M.R., Lavoie, S., Michaud, M., Blum, A.M., Tran, S.V., Weinstock, J.V., Gallini, C.A., Redding, K., Margolskee, R.F., Osborne, L.C., et al. (2016). Tuft cells, taste-chemosensory cells, orchestrate parasite type 2 immunity in the gut. Science 351, 1329-1333.

20. Kaser, A., Lee, A.H., Franke, A., Glickman, J.N., Zeissig, S., Tilg, H., Nieuwenhuis, E.E., Higgins, D.E., Schreiber, S., Glimcher, L.H., et al. (2008). XBP1 links ER stress to intestinal inflammation and confers genetic risk for human inflammatory bowel disease. Cell 134, 743-756.

21. Strober, W., and Ehrhardt, R.O. (1993). Chronic intestinal inflammation: an unexpected outcome in cytokine or T cell receptor mutant mice. Cell 75, 203205.

22. Mombaerts, P., Mizoguchi, E., Grusby, M.J., Glimcher, L.H., Bhan, A.K., and Tonegawa, S. (1993). Spontaneous development of inflammatory bowel disease in T cell receptor mutant mice. Cell 75, 274-282.

23. Barker, N., van Es, J.H., Kuipers, J., Kujala, P., van den Born, M., Cozijnsen, M., Haegebarth, A., Korving, J., Begthel, H., Peters, P.J., et al. (2007). Identification of stem cells in small intestine and colon by marker gene Lgr5. Nature 449, 1003-1007.

24. Yan, K.S., Janda, C.Y., Chang, J., Zheng, G.X.Y., Larkin, K.A., Luca, V.C., Chia, L.A., Mah, A.T., Han, A., Terry, J.M., et al. (2017). Non-equivalence of Wnt and Rspondin ligands during Lgr5+ intestinal stem-cell self-renewal. Nature 545, 238-242.

25. Barriga, F.M., Montagni, E., Mana, M., Mendez-Lago, M., Hernando-Momblona, X., Sevillano, M., Guillaumet-Adkins, A., Rodriguez-Esteban, G., Buczacki, S.J.A., Gut, M., et al. (2017). Mex3a Marks a Slowly Dividing Subpopulation of Lgr5+ Intestinal Stem Cells. Cell Stem Cell 20, 801-816 e807.

26. Sato, T., Vries, R.G., Snippert, H.J., van de Wetering, M., Barker, N., Stange, D.E., van Es, J.H., Abo, A., Kujala, P., Peters, P.J., et al. (2009). Single Lgr5 stem cells build crypt-villus structures in vitro without a mesenchymal niche. Nature 459, 262-265.

27. Ramilowski, J.A., Goldberg, T., Harshbarger, J., Kloppmann, E., Lizio, M., Satagopam, V.P., Itoh, M., Kawaji, H., Carninci, P., Rost, B., et al. (2015). A draft 
network of ligand-receptor-mediated multicellular signalling in human. Nat Commun 6, 7866.

28. Howie, D., Garcia Rueda, H., Brown, M.H., and Waldmann, H. (2013). Secreted and transmembrane $1 \mathrm{~A}$ is a novel co-stimulatory ligand. PLoS One 8, e73610.

29. Kambayashi, T., and Laufer, T.M. (2014). Atypical MHC class II-expressing antigen-presenting cells: can anything replace a dendritic cell? Nature reviews. Immunology 14, 719-730.

30. Thelemann, C., Eren, R.O., Coutaz, M., Brasseit, J., Bouzourene, H., Rosa, M., Duval, A., Lavanchy, C., Mack, V., Mueller, C., et al. (2014). Interferon-gamma induces expression of MHC class II on intestinal epithelial cells and protects mice from colitis. PLoS One 9, e86844.

31. Bland, P. (1988). MHC class II expression by the gut epithelium. Immunol Today 9, 174-178.

32. Salomon, P., Pizzimenti, A., Panja, A., Reisman, A., and Mayer, L. (1991). The expression and regulation of class II antigens in normal and inflammatory bowel disease peripheral blood monocytes and intestinal epithelium. Autoimmunity 9, 141-149.

33. Madsen, L., Labrecque, N., Engberg, J., Dierich, A., Svejgaard, A., Benoist, C., Mathis, D., and Fugger, L. (1999). Mice lacking all conventional MHC class II genes. Proc Natl Acad Sci U S A 96, 10338-10343.

34. Tirosh, I., Izar, B., Prakadan, S.M., Wadsworth, M.H., 2nd, Treacy, D., Trombetta, J.J., Rotem, A., Rodman, C., Lian, C., Murphy, G., et al. (2016). Dissecting the multicellular ecosystem of metastatic melanoma by single-cell RNA-seq. Science 352, 189-196.

35. Kowalczyk, M.S., Tirosh, I., Heckl, D., Rao, T.N., Dixit, A., Haas, B.J., Schneider, R.K., Wagers, A.J., Ebert, B.L., and Regev, A. (2015). Single-cell RNA-seq reveals changes in cell cycle and differentiation programs upon aging of hematopoietic stem cells. Genome Research 25, 1860-1872.

36. Patel, A.P., Tirosh, I., Trombetta, J.J., Shalek, A.K., Gillespie, S.M., Wakimoto, H., Cahill, D.P., Nahed, B.V., Curry, W.T., Martuza, R.L., et al. (2014). Single-cell RNA-seq highlights intratumoral heterogeneity in primary glioblastoma. Science 344, 1396-1401.

37. Hayami, S., Yoshimatsu, M., Veerakumarasivam, A., Unoki, M., Iwai, Y., Tsunoda, T., Field, H.I., Kelly, J.D., Neal, D.E., Yamaue, H., et al. (2010). Overexpression of the JmjC histone demethylase KDM5B in human carcinogenesis: involvement in the proliferation of cancer cells through the E2F/RB pathway. Molecular Cancer 9, 59-14.

38. Roesch, A., Fukunaga-Kalabis, M., Schmidt, E.C., Zabierowski, S.E., Brafford, P.A., Vultur, A., Basu, D., Gimotty, P., Vogt, T., and Herlyn, M. (2010). A Temporarily Distinct Subpopulation of Slow-Cycling Melanoma Cells Is Required for Continuous Tumor Growth. Cell 141, 583-594.

39. Oki, T., Nishimura, K., Kitaura, J., Togami, K., Maehara, A., Izawa, K., SakaueSawano, A., Niida, A., Miyano, S., Aburatani, H., et al. (2014). A novel cellcycle-indicator, mVenus-p27K-, identifies quiescent cells and visualizes G0G1 transition. Scientific Reports 4, 1-10. 
40. Habib, N., Li, Y., Heidenreich, M., Swiech, L., Avraham-Davidi, I., Trombetta, J.J., Hession, C., Zhang, F., and Regev, A. (2016). Div-Seq: Single-nucleus RNASeq reveals dynamics of rare adult newborn neurons. Science 353, 925-928.

41. Nozaki, K., Mochizuki, W., Matsumoto, Y., Matsumoto, T., Fukuda, M., Mizutani, T., Watanabe, M., and Nakamura, T. (2016). Co-culture with intestinal epithelial organoids allows efficient expansion and motility analysis of intraepithelial lymphocytes. J Gastroenterol 51, 206-213.

42. Jager, A., Dardalhon, V., Sobel, R.A., Bettelli, E., and Kuchroo, V.K. (2009). Th1, Th17, and Th9 effector cells induce experimental autoimmune encephalomyelitis with different pathological phenotypes. J Immunol 183, 7169-7177.

43. Sato, T., and Clevers, H. (2013). Growing self-organizing mini-guts from a single intestinal stem cell: mechanism and applications. Science 340, 11901194.

44. Grün, D., Lyubimova, A., Kester, L., Wiebrands, K., Basak, O., Sasaki, N., Clevers, H., and van Oudenaarden, A. (2015). Single-cell messenger RNA sequencing reveals rare intestinal cell types. Nature, 1-23.

45. Farin, H.F., Karthaus, W.R., Kujala, P., Rakhshandehroo, M., Schwank, G., Vries, R.G., Kalkhoven, E., Nieuwenhuis, E.E., and Clevers, H. (2014). Paneth cell extrusion and release of antimicrobial products is directly controlled by immune cell-derived IFN-gamma. J Exp Med 211, 1393-1405.

46. Gerbe, F., Sidot, E., Smyth, D.J., Ohmoto, M., Matsumoto, I., Dardalhon, V., Cesses, P., Garnier, L., Pouzolles, M., Brulin, B., et al. (2016). Intestinal epithelial tuft cells initiate type 2 mucosal immunity to helminth parasites. Nature 529, 226-230.

47. Beyaz, S., Mana, M.D., Roper, J., Kedrin, D., Saadatpour, A., Hong, S.J., BauerRowe, K.E., Xifaras, M.E., Akkad, A., Arias, E., et al. (2016). High-fat diet enhances stemness and tumorigenicity of intestinal progenitors. Nature 531, 53-58.

48. Hashimoto, K., Joshi, S.K., and Koni, P.A. (2002). A conditional null allele of the major histocompatibility IA-beta chain gene. Genesis 32, 152-153.

49. el Marjou, F., Janssen, K.P., Chang, B.H., Li, M., Hindie, V., Chan, L., Louvard, D., Chambon, P., Metzger, D., and Robine, S. (2004). Tissue-specific and inducible Cre-mediated recombination in the gut epithelium. Genesis 39, 186-193.

50. Cordier, A.C., and Haumont, S.M. (1980). Development of thymus, parathyroids, and ultimo-branchial bodies in NMRI and nude mice. Am J Anat 157, 227-263.

51. Mombaerts, P., Clarke, A.R., Rudnicki, M.A., Iacomini, J., Itohara, S., Lafaille, J.J., Wang, L., Ichikawa, Y., Jaenisch, R., Hooper, M.L., et al. (1992). Mutations in Tcell antigen receptor genes alpha and beta block thymocyte development at different stages. Nature 360, 225-231.

52. Kim, J.M., Rasmussen, J.P., and Rudensky, A.Y. (2007). Regulatory T cells prevent catastrophic autoimmunity throughout the lifespan of mice. Nat Immunol 8, 191-197. 
53. Liberzon, A., Subramanian, A., Pinchback, R., Thorvaldsdottir, H., Tamayo, P., and Mesirov, J.P. (2011). Molecular signatures database (MSigDB) 3.0. Bioinformatics 27, 1739-1740.

54. Ritsma, L., Ellenbroek, S.I., Zomer, A., Snippert, H.J., de Sauvage, F.J., Simons, B.D., Clevers, H., and van Rheenen, J. (2014). Intestinal crypt homeostasis revealed at single-stem-cell level by in vivo live imaging. Nature 507, 362365.

55. Snippert, H.J., van der Flier, L.G., Sato, T., van Es, J.H., van den Born, M., KroonVeenboer, C., Barker, N., Klein, A.M., van Rheenen, J., Simons, B.D., et al. (2010). Intestinal crypt homeostasis results from neutral competition between symmetrically dividing Lgr5 stem cells. Cell 143, 134-144.

56. Tanoue, T., Atarashi, K., and Honda, K. (2016). Development and maintenance of intestinal regulatory T cells. Nature reviews. Immunology 16, 295-309.

57. Ivanov, II, Atarashi, K., Manel, N., Brodie, E.L., Shima, T., Karaoz, U., Wei, D., Goldfarb, K.C., Santee, C.A., Lynch, S.V., et al. (2009). Induction of intestinal Th17 cells by segmented filamentous bacteria. Cell 139, 485-498. 


\section{Figure 1}

A
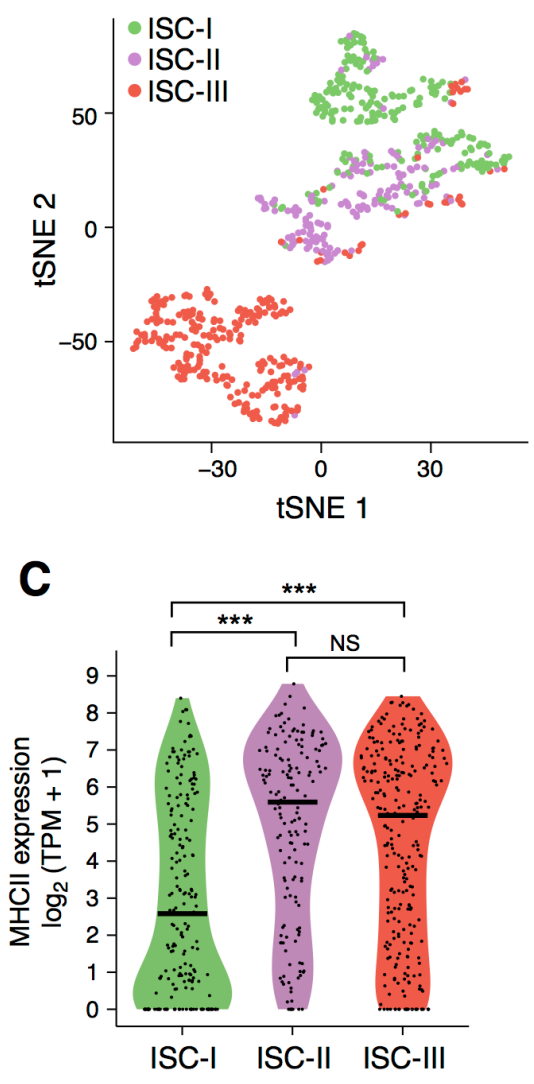

B

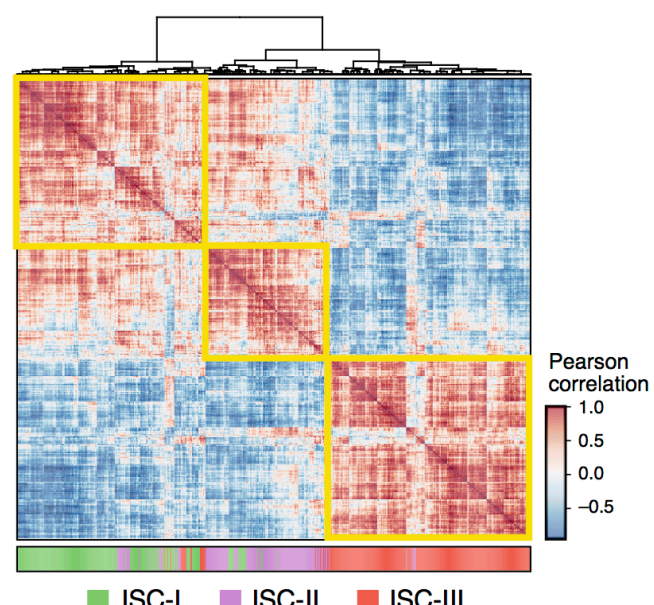

D

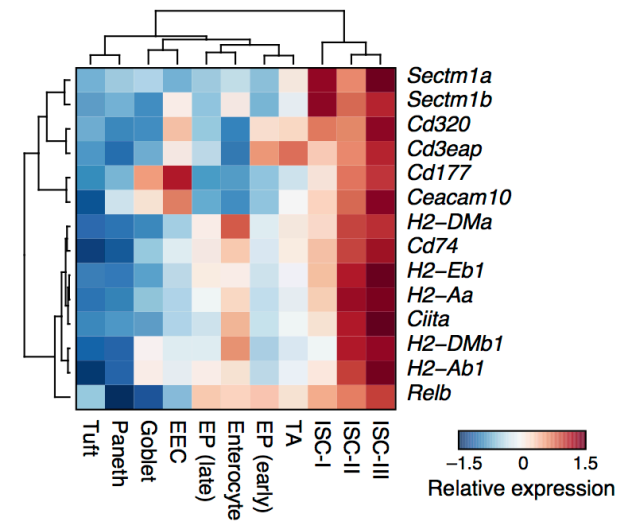

E

I-A/I-E
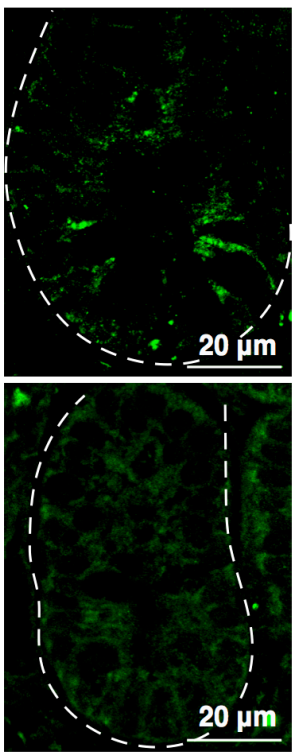

Lyz1
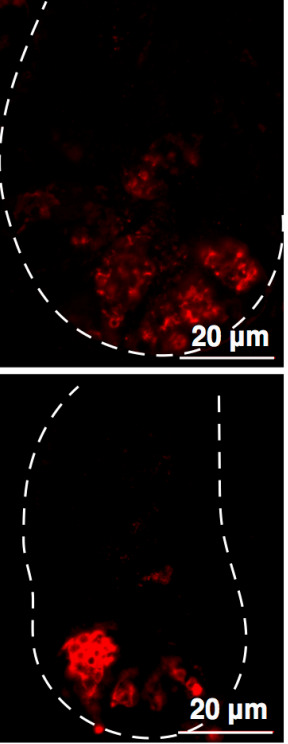

Merge

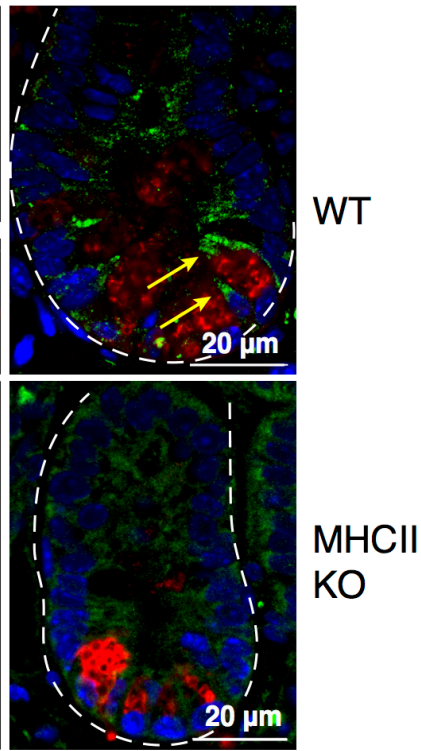




\section{Figure 2}
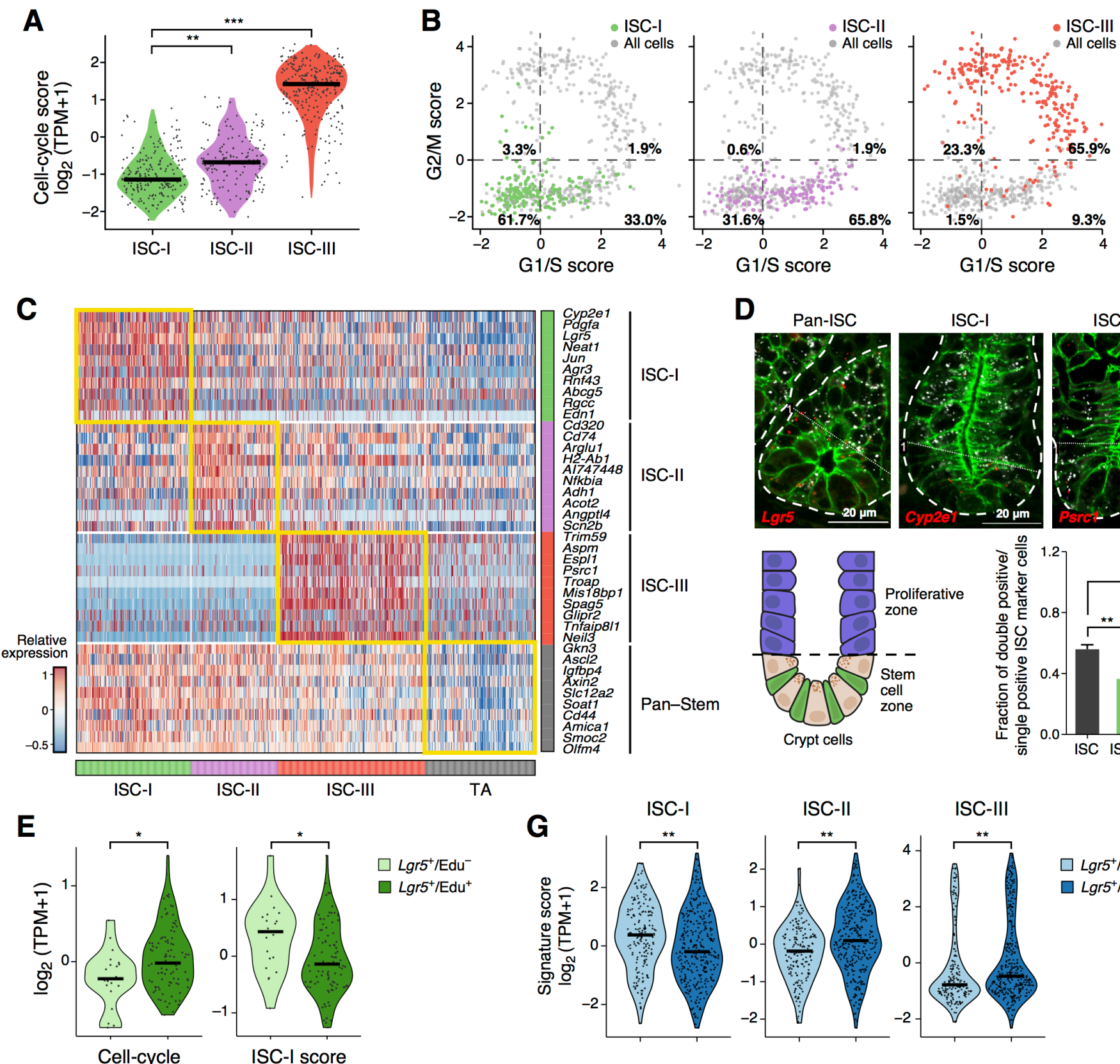

D
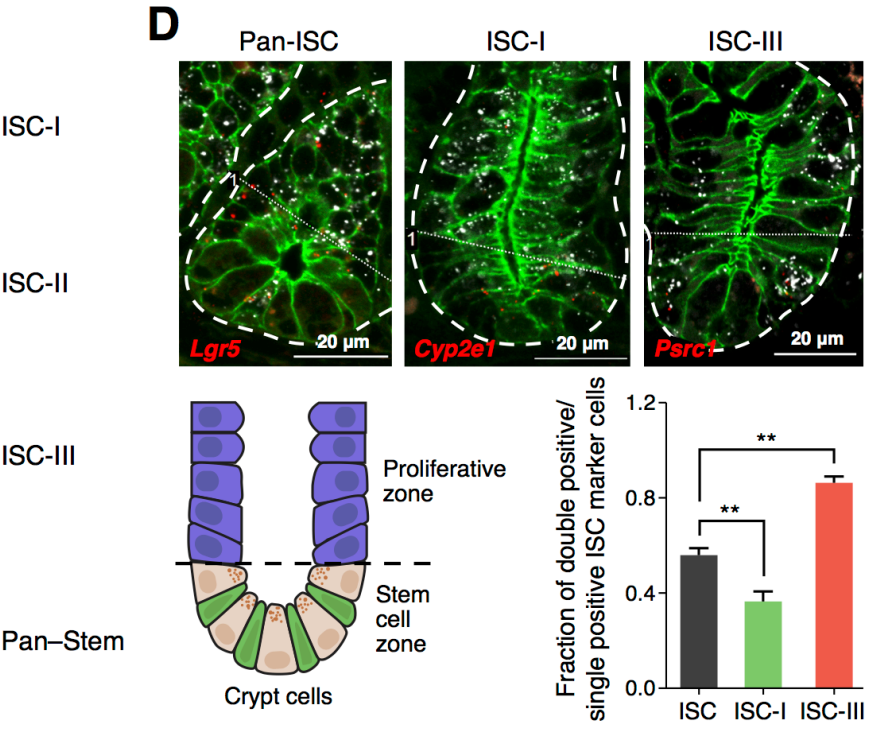

ISC-I

ISC-II

ISC-III
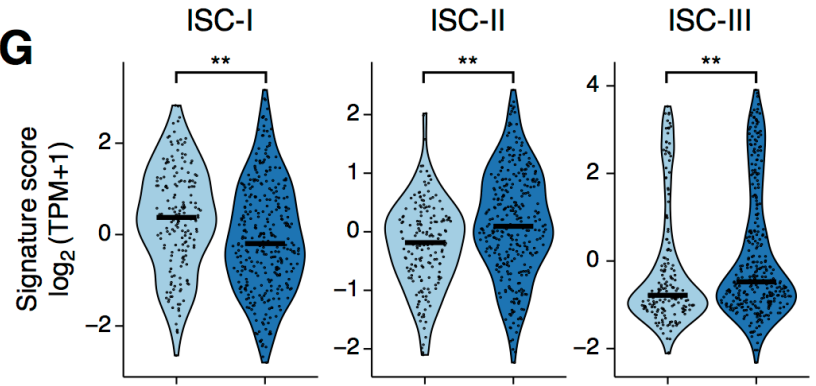

$\mathbf{F}$
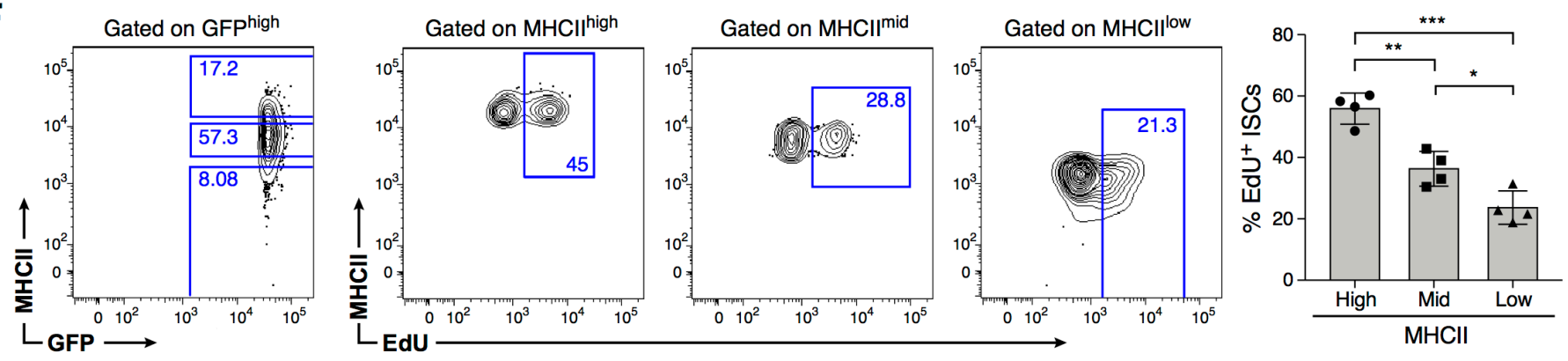


\section{Figure 3}

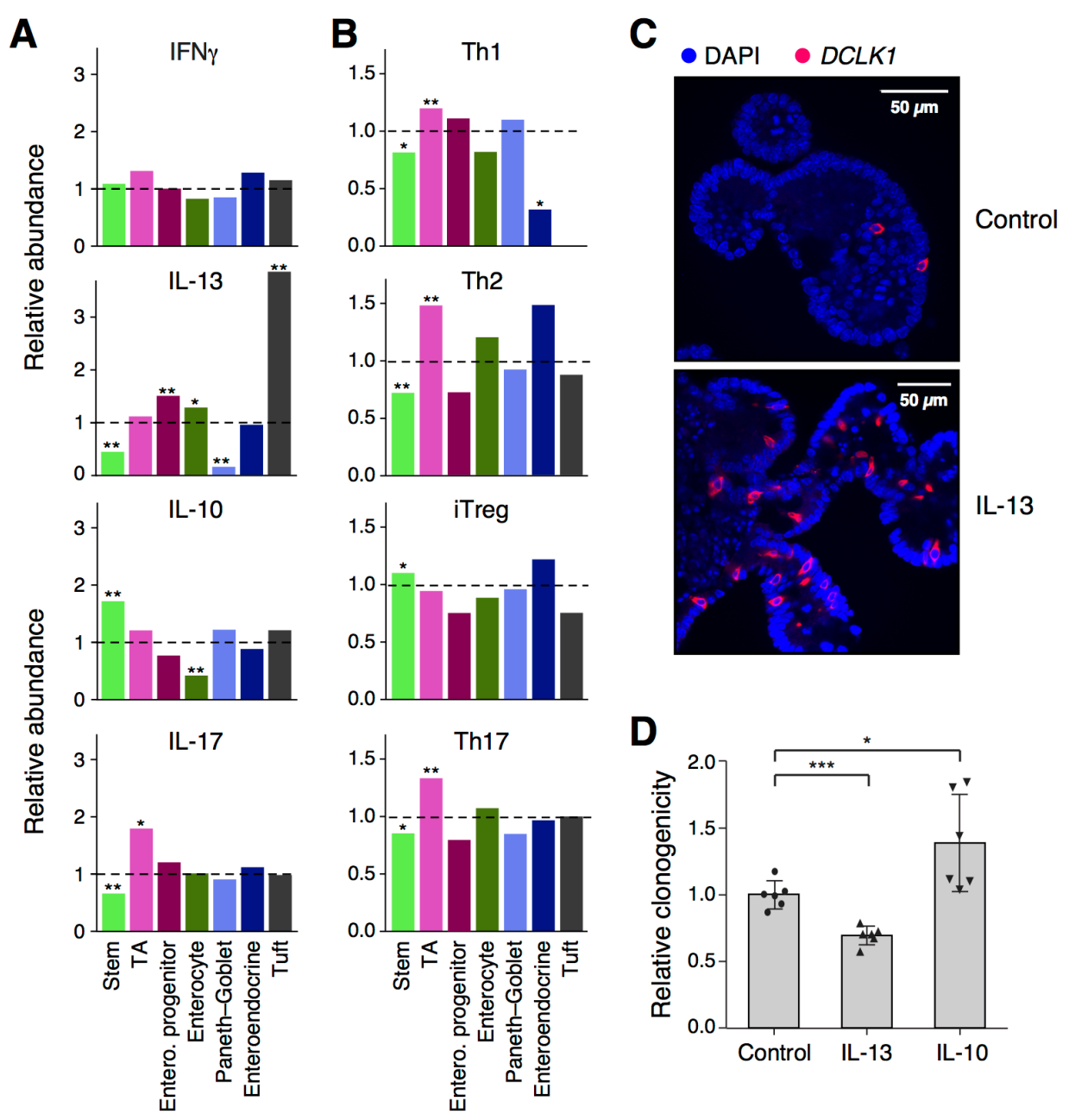




\section{Figure 4}
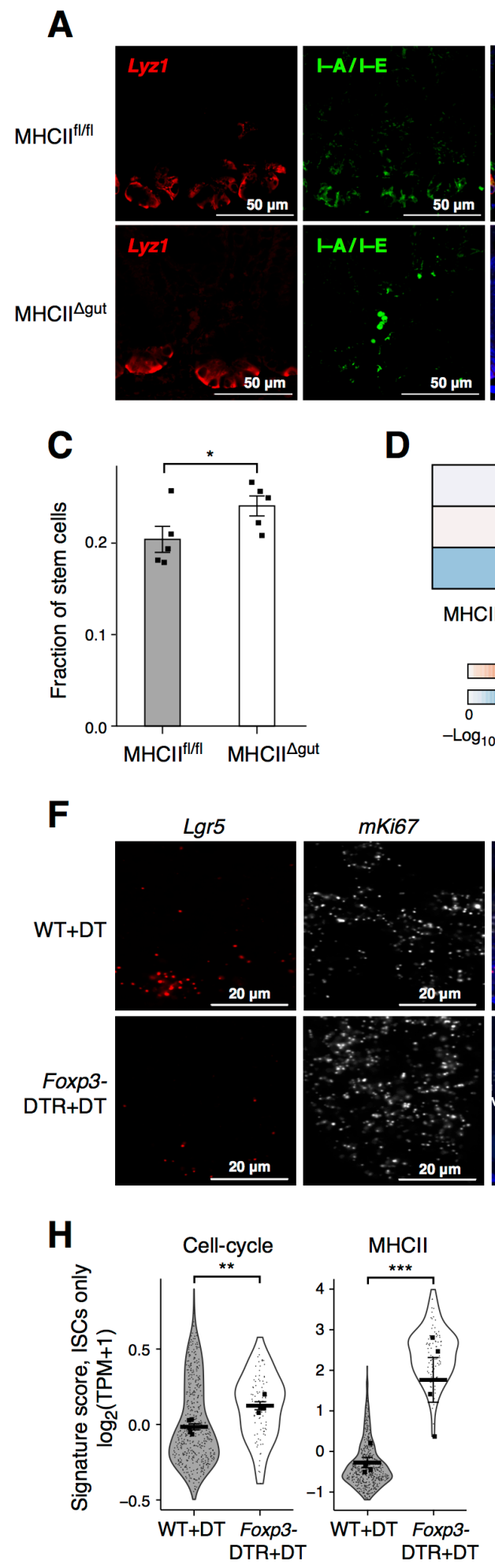

B
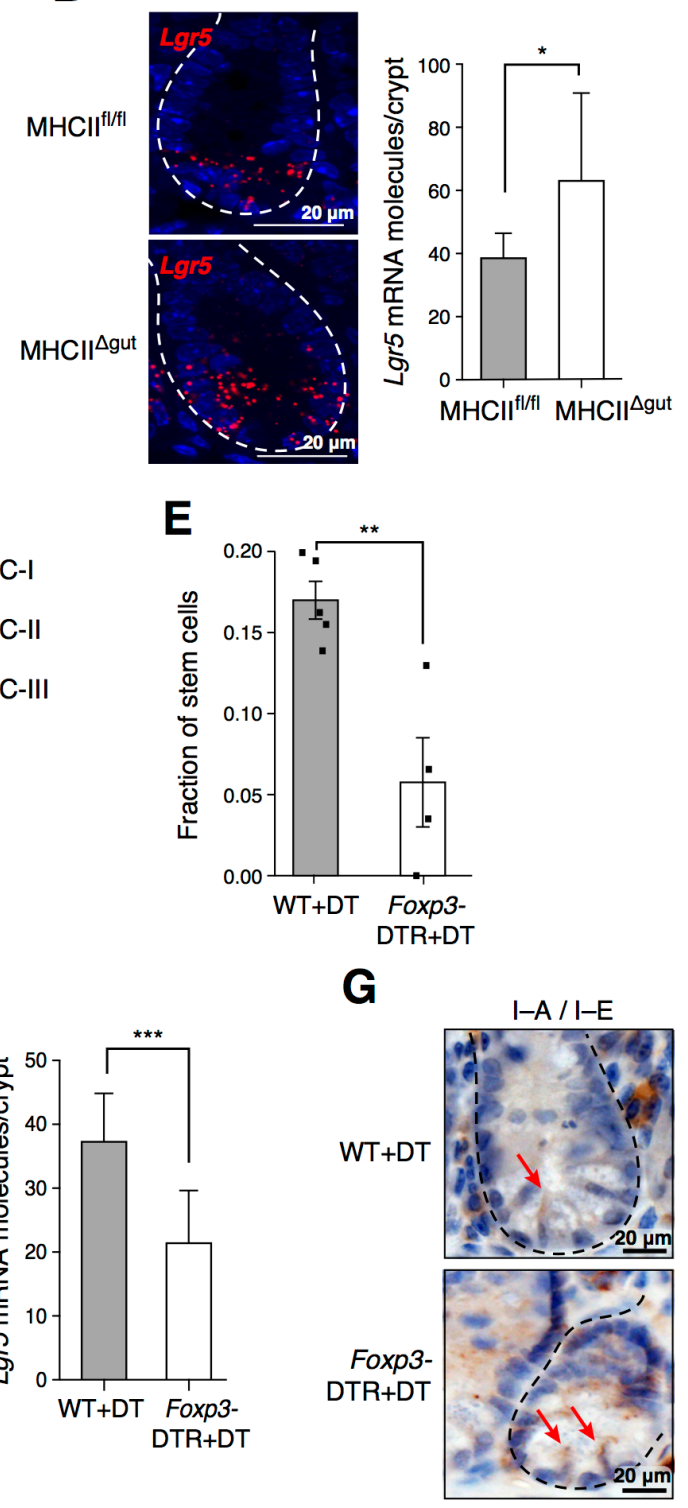


\section{Supplementary Materials for}

\section{T helper cells modulate intestinal stem cell renewal and differentiation}

\section{Materials and Methods:}

\section{Experimental Methods:}

\section{Mice}

All mouse work was performed in accordance with the Institutional Animal Care and Use Committees (IACUC) and relevant guidelines at the Broad Institute and MIT, with protocols 0055-05-15 and 0612-058-15, respectively. Seven to ten weeks old female or male C57BL/6J wild-type, Lgr5-EGFP-IRES-CreER ${ }^{T 2}$ (Lgr5-GFP), MHCII-KO, Foxp3DTR, B6 Nude and TCRß-KO mice were obtained from the Jackson Laboratory (Bar Harbor, ME), while MHCII-EGFP mice were obtained from Hidde Ploegh's lab. All mice were housed under specific-pathogen-free (SPF) conditions at either the Broad Institute or MIT animal facilities; infection experiments were conducted at the laboratory of Dr. HN Shi, maintained under specific pathogen-free conditions at Massachusetts General Hospital (Charlestown, MA), with protocol 2003N000158.

BrdU and EDU incorporation: EdU was injected intraperitoneally (IP) into Lgr5-GFP mice at $100 \mathrm{mg} \mathrm{kg}^{-1}$ for 2 or 4 hours before tissue collection.

S. enterica and $H$. polygyrus infection. C57BL/6J mice (Jackson Laboratory) were infected with 200 third-stage larvae of $H$. polygyrus or $10^{8}$ S. enterica. H. polygyrus was propagated as previously described [58]. Mice were sacrificed 3 and 10 days after $H$. polygyrus infection. For the MHCII blocking experiment, mice infected with $H$. polygyrus 
were injected with $500 \mu \mathrm{g}$ of blocking anti-mouse MHCIl antibody (BioXCell) or Rat lgG2b isotype control (BioXCell) one-day prior to and for 2 consecutive days after $H$. polygyrus infection. For $S$. enterica infection, mice were infected with a naturally streptomycin-resistant SL1344 strain of S. Typhimurium $\left(10^{8}\right.$ cells) as previously described [59] and were sacrificed 48 hours after infection.

Foxp3-DTR. Foxp3 and wild-type C57BL/6J mice were injected intraperitoneally with diphtheria toxin (DT) at $22.5 \mathrm{ng} / \mathrm{g}$ body weight every other day for one week and then sacrificed.

MHCIl deletion in intestinal epithelial cells. Cre activity was induced in 7-10 weeks old mice by intraperitoneal injection (IP) of Tamoxifen (SIGMA), diluted in corn oil, 4 mg per injection, 3 times, every other day. Mice were sacrificed 10 days after the first injection.

\section{Cell dissociation and crypt isolation}

Crypt isolation. For all mice, crypts were isolated from the whole small intestine or the duodenum, jejunum and ileum compartments to account for regional distribution of Lgr5 $^{+}$stem cells. The small intestine was extracted and rinsed in cold PBS. The tissue was opened longitudinally and sliced into small fragments roughly $0.2 \mathrm{~cm}$ long. The tissue was incubated in 20mM EDTA-PBS on ice for $90 \mathrm{~min}$, while shaking every 30 min. The tissue was then shaken vigorously and the supernatant was collected as fraction 1 in a new conical tube. The tissue was incubated in fresh EDTA-PBS and a new fraction was collected every $30 \mathrm{~min}$. Fractions were collected until the supernatant 
consisted almost entirely of crypts. The final fraction (enriched for crypts) was washed twice in PBS, centrifuged at $300 \mathrm{~g}$ for $3 \mathrm{~min}$, and dissociated with TrypLE Express (Invitrogen) for $1 \mathrm{~min}$ at $37^{\circ} \mathrm{C}$. The single-cell suspension was then passed through a $40 \mu \mathrm{m}$ filter and stained for fluorescence-activated cell sorting (FACS) machine (Astrios) sorting for either scRNA-seq method (below).

Immune cell isolation. Immune cells from the Lamina Propria were isolated enzymatically by incubating the small intestine with Liberase TM (100 $\mu \mathrm{g} / \mathrm{mL}$, Sigma) and DNasel $\left(100 \mu \mathrm{g} / \mathrm{mL}\right.$, Sigma) for $30 \mathrm{~min}$ at $37^{\circ} \mathrm{C}$. Immune cells were also isolated from the mesenteric lymph nodes $(\mathrm{mLN})$. Cells were then incubated with CD3, CD4, CD45, or CD11b FACS-labeled antibodies and sorted for scRNA-seq.

\section{Cell sorting}

Plate-based, full-length scRNA-seq. FACS (Astrios) was used to sort one single cell into each well of a 96-well PCR plate containing $5 \mu$ of TCL buffer with $1 \% 2$ mercaptoethanol. The cells were stained for 7AAD ${ }^{-}$(Life Technologies), CD45(eBioscience), CD31- (eBioscience), Ter119- (eBioscience), EpCAM ${ }^{+}$(eBioscience), and for specific epithelial cells were also stained for $\mathrm{CD}_{2}{ }^{+/-}$(eBioscience) and $\mathrm{c}-\mathrm{Kit}^{+/-}$ (eBioscience). To enrich for specific IEC populations, cells were isolated from Lgr5-GFP mice, stained with the antibodies mentioned above and gated for GFP-high (stem cells), GFP-low (TAs), GFP $/ \mathrm{CD}^{2} 4^{+/} / \mathrm{c}-\mathrm{Kit}^{+/-}$(secretory lineages) or $\mathrm{GFP}^{-} / \mathrm{CD}^{2} 4^{-} / \mathrm{EpCAM}^{+}$ (epithelial cells). A population control of 200 cells was sorted into one well and a no-cell control was sorted into another well. After sorting, the plate was sealed tightly with a 
Microseal $\mathrm{F}$ and centrifuged at $800 \mathrm{~g}$ for $1 \mathrm{~min}$. The plate was immediately frozen on dry ice and kept at $-80^{\circ} \mathrm{C}$ until ready for the lysate cleanup. Bulk population cells were sorted into an Eppendorf tube containing $100 \mu$ solution of TCL with $1 \% 2-$ mercaptoethanol and stored at $-80^{\circ} \mathrm{C}$.

Droplet-based scRNA-seq. Cells were sorted with the same parameters as described for plate-based scRNA-seq, but into an Eppendorf tube containing $50 \mu \mathrm{l}$ of $0.4 \%$ BSAPBS and stored on ice until proceeding to the GemCode Single Cell Platform or the Chromium Single Cell 3' Library.

\section{Plate-based scRNA-seq}

Single cells. Libraries were prepared using a modified SMART-Seq2 protocol as previously reported [60]. Briefly, RNA lysate cleanup was performed using RNAClean XP beads (Agencourt), followed by reverse transcription with Maxima Reverse Transcriptase (Life Technologies) and whole transcription amplification (WTA) with KAPA HotStart HIFI 2× ReadyMix (Kapa Biosystems) for 21 cycles. WTA products were purified with Ampure XP beads (Beckman Coulter), quantified with Qubit dsDNA HS Assay Kit (ThermoFisher), and assessed with a high sensitivity DNA chip (Agilent). RNA-seq libraries were constructed from purified WTA products using Nextera XT DNA Library Preparation Kit (Illumina). On each plate, the population and no-cell controls were processed using the same method as the single cells. The libraries were sequenced on an Illumina NextSeq 500. 


\section{Droplet-based scRNA-seq}

Single cells were processed through the GemCode Single Cell Platform using the GemCode Gel Bead, Library and Chip Kits, or the Chromium Single Cell 3' Library, Gel Bead and Chip Kits (10X Genomics, Pleasanton, CA), following the manufacturer's protocol. Briefly, an input of 6,000 cells was added to each channel of a chip with a recovery rate of 1,500 cells. The cells were then partitioned into Gel Beads in Emulsion (GEMs) in the GemCode instrument, where cell lysis and barcoded reverse transcription of RNA occurred, followed by amplification, shearing and 5' adaptor and sample index attachment. Libraries were sequenced on an Illumina NextSeq 500.

\section{Div-Seq}

Lgr5-GFP mice were intraperitoneally (IP) injected with $100 \mathrm{mg} \mathrm{kg}^{-1}$ EdU (Click-iT Plus EdU Pacific Blue Flow Cytometry Assay Kit, Thermo Fisher Scientific) for 2 hours and then sacrificed. Crypts were isolated as described above and Lgr $5^{\text {High }}$ cells were FACS sorted into PBS, spun down to remove the supernatant, flash frozen and stored in $80^{\circ} \mathrm{C}$. Nuclei were then isolated using EZ Prep NUC-101 (Sigma) per manufacturer's recommendation, and then incubated in the Click-iT Cocktail per manufacturer's recommendations for $30 \mathrm{~min}$, washed in 1\% BSA-PBS and counterstained with Vybrant DyeCyle Ruby stain (Thermo Fisher Scientific) for 15 min. Nuclei were then individually sorted into the wells of 96 well plates with $\mathrm{TCL}+1 \% 2$-mercaptoethanol as described before [61] using FACS, based on positive Ruby and either EdU ${ }^{\text {high }}$ or EdU ${ }^{\text {low }}$. Platebased single-nucleus RNA-seq (snRNA-Seq) was then performed as described above for scRNA-seq. 


\section{Immunofluorescence and single-molecule fluorescence in situ hybridization (smFISH)}

Immunofluorescence (IFA) and immunohistochemistry (IHC): Staining of small intestinal tissue was conducted as previously described [62]. Briefly, tissues were fixed for 14 hours in formalin, embedded in paraffin and cut into $5 \mu \mathrm{m}$ thick sections. Sections were deparaffinized with standard techniques, incubated with primary antibodies overnight at $4^{\circ} \mathrm{C}$, and then incubated with secondary antibodies at room temperature for $30 \mathrm{~min}$. Slides were mounted with Slowfade Mountant+DAPI (Life Technologies, S36964) and sealed.

Single-molecule fluorescence in situ hybridization (smFISH): RNAScope Fluorescent Multiplex and RNAScope Multiplex Fluorescent v2 (Advanced Cell Diagnostics) were used per manufacturer's recommendations with the following alterations. Target Retrieval boiling time was adjusted to 12 minutes and incubation with Protease IV at $40^{\circ} \mathrm{C}$ was adjusted to 8 minutes. Slides were mounted with Slowfade Mountant+DAPI (Life Technologies, S36964) and sealed.

Combined IFA and smFISH was implemented by first performing $\mathrm{smFISH}$, as described above, with the following alterations. After Amp 4, tissue sections were washed in washing buffer, incubated with primary antibodies overnight at $4^{\circ} \mathrm{C}$, washed in $1 \times$ TBST 3 times and then incubated with secondary antibodies for $30 \mathrm{~min}$ at room temperature. 
Slides were mounted with Slowfade Mountant+DAPI (Life Technologies, S36964) and sealed.

\section{Image analysis}

Image acquisition. Images of tissue sections were taken with a confocal microscope Fluorview FV1200 using Kalman and sequential laser emission to reduce noise and signal overlap. Scale bars were added to each image using the confocal software FV10ASW 3.1 Viewer. Images were overlaid and visualized using ImageJ software [63].

Quantification of proliferating stem cells. Combined IFA and smFISH images of wildtype C57BL/6J small intestinal tissues were assessed by staining for E-Cadherin to mark cell borders, the canonical proliferation marker mKi67, and either the common ISC marker Lgr5, our predicted IcISC markers (Cyp2e1 or Fgfr4) or our predicted hclSC markers (Psrc1 or Cenpf). A line was drawn to establish the bottom of the crypt, termed "stem cell zone", and quantification was only assessed within that zone. For each ISC subset marker, more than 10 randomly chosen intact crypts were analyzed. Cells were examined by double blind quantification and were determined double positive if they coexpressed mKi67 and one of the ISC subset markers. Proliferating cells in each ISC subset was measured by calculating the fraction of double positive cells out of all cells positive for the specific ISC subset marker.

Automated quantification of Lgr5 mRNA molecules in smFISH images of intestinal crypts within different mouse models (Figure 4 and Figure S9) was performed using a 
custom Python script.

\section{Antibodies and probes}

Antibodies used for immunofluorescence. Rabbit anti-DCLK1 (1:200, Abcam GR245168-1), mouse anti-E-cadherin (1:100, BD Biosciences 610181), rat antiLysozyme (Dako, A0099) and anti-mouse I-A/I-E (1:100, Biolegend 107601). Alexa

Fluor 488-, 594-, and 647-conjugated secondary antibodies were used (Life Technologies).

Probes used for single-molecule RNAscope (Advanced Cell Diagnostics): Lgr5 (C1,C3), Cyp2e1 (C2), Psrc1 (C1), Fgfr4 (C2), Cenpf (C3), mKi67 (C1,C3).

\section{Th cell polarization in vitro}

$\mathrm{CD}^{+}$naïve $\left(\mathrm{CD} 44^{\mathrm{lo}} \mathrm{CD} 62 \mathrm{~L}^{+} \mathrm{CD} 25^{-}\right) \mathrm{T}$ cells were isolated from spleen and lymph nodes of 7-10 weeks old C57BL/6J mice using flow cytometry cell sorting. The purity of isolated T cell populations routinely exceeded $98 \%$. Naïve T cells were stimulated with plate-bound anti-CD3 $(145-2 \mathrm{C} 11,1 \mathrm{mg} / \mathrm{ml})$ and anti-CD28 $(\mathrm{PV}-1,1 \mathrm{mg} / \mathrm{ml})$ and polarizing cytokines (Th1: 4 ng/ml IL-12; Th2: 4 ng/ml IL-4; Th17: 10 ng/ml IL-6, 2 ng/ml TGF- $\beta 1$; $\mathrm{iT}_{\text {reg: }} 5 \mathrm{ng} / \mathrm{ml}$ TGF- $\beta 1$; all cytokines from R\&D).

\section{Intestinal organoid cultures}

Organoid cultures. Following crypt isolation from the whole small intestine [64], the single-cell suspension was re-suspended in Matrigel (BD Bioscience) with $1 \mu \mathrm{M}$ Jagged- 
1 peptide (Ana-Spec). Roughly 300 crypts embedded in $25 \mu \mathrm{l}$ of Matrigel were seeded onto each well of a 24-well plate. Once solidified, the Matrigel was incubated in $600 \mu \mathrm{l}$ culture medium (Advanced DMEM/F12, Invitrogen) with streptomycin/penicillin and glutamatax and supplemented with EGF (100 ng/mL, Peprotech), R-Spondin-1 (600ng/mL, R\&D), Noggin (100ng/mL, Prepotech), Y-276432 dihydrochloride

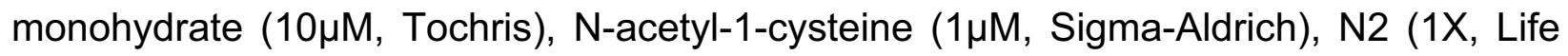
Technologies), B27 (1X, Life Technologies) and Wnt3A (25ng/mL, R\&D Systems). Fresh media was replaced on day 3 , and organoids were passaged by dissociation with TrypLE and re-suspended in new Matrigel on day 6 with a 1:3 split ratio.

$T$ helper cell co-culture experiments. Organoids were cultured with Th1, Th2, Th17 or $\mathrm{iT}_{\text {regs. }}$ Roughly $10,000 \mathrm{~T}$ helper cells were added to each well of 500 organoids and were supplemented either to the medium or suspended in the Matrigel. Treated organoids were dissociated and subjected to droplet based scRNA-seq.

Cytokine treated organoids. Organoids were additionally treated with $0.5 \mathrm{u} / \mathrm{ml} \mathrm{IFNy}, 20$ $\mathrm{ng} / \mathrm{ml} \mathrm{IL-13,} 20 \mathrm{ng} / \mathrm{ml} \mathrm{IL-17A}$ or $10 \mathrm{ng} / \mathrm{ml} \mathrm{IL-10} \mathrm{in} \mathrm{the} \mathrm{culture} \mathrm{medium} \mathrm{for} 3$ days.

Re-seeding after cytokine treatment. 500 organoids/well were treated with cytokines, as in the cytokine treated organoids above, collected after 3 days and then re-seeded at 500 organoids/well in fresh media without cytokines. Each day, images were taken at $2 \mathrm{X}$ magnification and quantification of organoids number was performed with the ImageJ software. 


\section{Two-photon intra-vital microscopy (2P-IVM) of T cells and ISCs}

To generate gut-homing $\mathrm{T}$ cells visualized by $2 \mathrm{P}-\mathrm{IVM}$, a combination of modified protocols $[65,66]$ was used. $\mathrm{CD}^{+} \mathrm{T}$ cells were isolated from spleen, $\mathrm{pLN}$ and $\mathrm{mLN}$ from $\beta$-actin-RFP mice using a MACS CD4 T cell positive-selection kit (Miltenyi clone L3T4) following the manufacturer's instructions. Plates were pre-treated with $5 \mu \mathrm{g} / \mathrm{mL}$ anti-CD3 (clone 145-2C11) and $1 \mu \mathrm{g} / \mathrm{mL}$ anti-CD28 (clone 37.51) and $1 \times 10^{6} \mathrm{CD}^{+} \mathrm{T}$ cells were added to each well for a final volume of $2.5 \mathrm{~mL}$ in complete RPMI1640 media supplemented with all-trans Retinoic Acid (100nM, Sigma R2625). The T cells were cultured for 96 hours before replacing half of the volume with fresh media containing $20 \mathrm{U} / \mathrm{mL}$ of rIL-2 and then cultured for another 48 hours. Before adoptive transfer into Lgr5-GFP hosts, the gut-homing phenotype was validated with flow cytometry for $\alpha 4 \beta 7$ and CCR9 expression. $1 \times 10^{7}$ cells were then transferred into recipient mice for two hours, and treated with 20ug of anti-CD3 (clone 2C11). 2P-IVM was performed 72 hours following transfer. The small intestine was surgically exposed through a laparotomy incision. Anesthetized mice were placed on a custom-built stage with a loop of the intact small intestine fixed to a temperature-controlled metallic support to facilitate exposure of the serosal aspect to a water-immersion 20X objective ( 0.95 numerical aperture) of an upright microscope (Prairie Technologies). A Mai Tai Ti:sapphire laser (SpectraPhysics) was tuned between $870 \mathrm{~nm}$ and $900 \mathrm{~nm}$ for multiphoton excitation and secondharmonic generation. For dynamic analysis of cell interaction in four dimensions, several $X / Y$ sections (512x512) with $Z$ spacing ranging from $2 \mu \mathrm{m}$ to $4 \mu \mathrm{m}$ were acquired every $15-20$ seconds with an electronic zoom varying from $1 X$ to $3 X$. Emitted light and 
second-harmonic signals were directed through 450/80-nm, 525/50-nm and 630/120-nm band-pass filters and detected with non-descanned detectors. Post-acquisition image analysis, volume-rendering and four-dimensional time-lapse videos were performed using Imaris software (Bitplane scientific software).

\section{Computational Methods:}

\section{Pre-processing of plate-based scRNA-seq data}

BAM files were converted to merged, de-multiplexed FASTQs using the Illumina provided Bcl2Fastq software package v2.17.1.14. Paired-end reads were mapped to the UCSC mm10 mouse transcriptome using Bowtie [67] with parameters "-q --phred33quals -n 1 -e 99999999 -I 25 -I 1 -X 2000 -a -m 15 -S -p 6", which allows alignment of sequences with one mismatch. Expression levels of genes were quantified as transcriptper-million (TPM) values calculated by RSEM [68] v1.2.3 in paired-end mode. For each cell, we quantified the number of genes for which at least one read was mapped, and then excluded all cells with either fewer than 3,000 detected genes or a transcriptomemapping rate of less than $40 \%$.

Selection of variable genes was performed by fitting a generalized linear model to the relationship between the squared coefficient of variation $(\mathrm{CV})$ and the mean expression level in log/log space, and selecting genes that significantly deviated $(p<0.05)$ from the fitted curve, as previously described [69]. 
For re-analysis of published data [70] (Figure S5F) normalized transcript counts were downloaded directly from the published data tables. Cell-quality filtering, transcript count normalization, tSNE, and clustering using the RacelD algorithm [70], were all performed using R scripts published online by the authors, using all default settings.

\section{Pre-processing of droplet-based scRNA-seq data}

De-multiplexing, alignment to the mm10 mouse transcriptome and UMI-collapsing were performed using the Cellranger toolkit (version 1.0.1) provided by 10X Genomics. For each cell, we quantified the number of genes for which at least one read was mapped, and then excluded all cells with fewer than 800 detected genes. Expression values $E_{i, j}$ for gene $i$ in cell $j$ were calculated by dividing UMI count values for gene $i$ by the sum of the UMI counts in cell $j$, to normalize for differences in coverage, and then multiplying by 10,000 to create TPM-like values, and finally calculating $\log _{2}(T P M+1)$ values. Batch correction was performed using ComBat [71] as implemented in the R package sva [72], using the default parametric adjustment mode. The output was a corrected expression matrix, which was used as input to further analysis. We identified highly variable genes as described above.

\section{Dimensionality reduction by PCA}

We restricted the expression matrix to the subsets of variable genes and high-quality cells noted above, and values were centered and scaled before input to PCA, which was implemented using the $\mathrm{R}$ function 'prcomp' from the 'stats' package for the plate- 
based dataset. For the droplet-based data, we used a randomized approximation to PCA [73], implemented using the 'rpca' function from the 'rsvd' $R$ package, with the parameter $k$ set to 100 . This low-rank approximation is several orders of magnitude faster to compute for very wide matrices. After PCA, significant PCs were identified using a permutation test as previously described [74], implemented using the 'permutationPA' function from the 'jackstraw' $R$ package. Only scores from these significant PCs were used as the input to further analysis.

\section{tSNE visualization}

For visualization purposes only (and not for clustering), dimensionality was further reduced using the Barnes-Hut approximate version of the $t$-distributed stochastic neighbor embedding (tSNE) $[75,76]$. This was implemented using the 'Rtsne' function from the 'Rtsne' R package using 20,000 iterations and a perplexity setting that ranged from 10 to 30 depending on the size of the dataset. Scores from the first $n$ PCs were used as the input to tSNE, where $n$ was determined for each dataset using the permutation test described above.

\section{Removing doublets}

In the plate-based dataset, several cells were outliers in terms of library complexity, which could possibly correspond to more than one individual cell per sequencing library, or 'doublets'. As a precaution, we removed any cells in the top quantile $1 \%$ of the distribution of genes detected per cell, as these may correspond to doublets. 


\section{$k-N N$ graph based clustering}

To cluster single cells by their expression profiles, we used unsupervised clustering, based on the Infomap graph-clustering algorithm [77], following approaches recently described for single-cell CyTOF data [78] and scRNA-seq [79]. Briefly, we constructed a $k$ nearest neighbor $(k-N N)$ graph on the data using as the metric for each pair of cells, the distance between the scores of significant PCs. The parameter $k$ was chosen in a manner roughly consistent with the size of the dataset (Table S1). Specifically, $k$ was set to 600,200 and 50 for the droplet dataset of $23,177,4,332$ and 1,090 cells from combined $\mathrm{T}$ cell and cytokines (Figure S5D), IL-13-treated and Th1 co-cultured organoids, respectively. For in vivo mouse models (Figure S8B), $k$ was set to 100, 300, 175, and 100 for nude mice, TCR $\beta$ KO, Foxp3-DTR and $\mathrm{MHCII}^{\Delta \text { gut }}$ respectively. For sub-clustering of stem cell subsets, we used $k=150$ and $k=40$ for the 637 and 123 Lgr $^{+}$ stem cells from our plate-based and the previously published [70] datasets, respectively. The combined Salmonella and H. polygyrus infection dataset (Figure S4BD) contained 5,122 immune cells and $k$ was set to 200 . The $k$-NN graph was computed using the function 'nng' from the R package 'cccd' and was then used as the input to Infomap [77], implemented using the 'infomap.community' function from the 'igraph' $R$ package.

Detected clusters were annotated by cell types or states using known markers for IEC subtypes [80]. Specifically, for each known epithelial type we selected five canonical marker genes (e.g., Lgr5, Ascl2, Slc12a2, Axin2 and Olfm4 for stem cells, or Lyz1, Defa17, Defa22, Defa24 and Ang4 for Paneth cells), and scored all clusters for their 
expression (see below for signature scoring procedure). In all cases, one cluster unambiguously expressed each cell-type signature, with two exceptions: in the platebased dataset, two clusters both expressed high levels of ISC markers (Figure S1A) and accordingly were merged to form a 'Stem' cluster and two other clusters were merged to form a 'TA' cluster based on high expression of cell-cycle genes and low-tomoderate expression of ISC genes.

\section{Assigning the three ISC states to region of origin using supervised classification}

To study the anatomical distribution of ISCs in different parts of the small intestine, we used a classification approach. First, we developed a classifier for the anatomical origin of ISCs using single-cell expression profiles of 2,965 ISCs extracted from duodenum, jejunum and ileum [81], by compiling a discriminative feature set using the expression levels of all genes differentially expressed (FDR $<0.1$, Mann-Whitney U-test, $\log _{2}$ foldchange $>0.25$ ) between stem cells from the three regions, and also the scores along the first 25 PCs. A 'random forest' classifier was trained on these features, and subsequently distinguished between ISCs from the three regions with an average outof-bag accuracy of $92.9 \%$. Finally, we used the trained classifier to classify the 637 ISCs

(Figure 1) and infer the fraction of cells drawn from each intestinal region found in each ISC state (Figure S1F).

\section{Cell-cell similarity matrix}

To visualize heterogeneity of ISCs within the 'Stem' cluster (637 cells), cell-cell similarities were computed. Principal component (PC) scores for each cell were 
computed across the 637 cells using the R function 'prcomp' as described above. The distance between cell $i$ and $j$ was calculated as the Pearson correlation between the scores of these two cells along the first 10 PCs. This distance matrix was then hierarchically clustered using Ward's method, implemented using the R function 'hclust' (with the 'method' argument set to 'ward.D2'), and visualized as a heatmap using the R function ‘aheatmap' (Figure 1B).

\section{Cell-cycle and ISC subset signatures}

To identify maximally specific genes associated with the three ISC subsets, we performed differential expression tests between each possible pairwise comparison between clusters. To ensure specificity of the detected marker genes to stem cells, the set of clusters included both the three ISC subsets (3 clusters), and all other detected IEC clusters (8 clusters; Figure S1A, right) for a total of 11 clusters. Then, for a given cluster, putative signature genes were filtered using the maximum FDR Q-value and ranked by the minimum $\log _{2}$ (fold-change). The minimum fold-change and maximum $Q$ value represents the weakest effect-size across all pairwise comparisons, therefore this is a stringent criterion. ISC subset signatures (Table S2) were obtained using a maximum FDR of 0.25 and a minimum $\log _{2}$ (fold-change) of 0.25 . To exclude the explicit effect of known cell-cycle genes on the gene signature of the ISC subsets we filtered out any gene annotated as directly participating in cell-cycle regulation. Annotated cellcycle genes were downloaded from the gene ontology (GO): http://amigo.geneontology.org/amigo/term/GO:0007049, and any gene appearing on this list was removed from the signature gene sets. 
Gene sets associated with G1/S and G2/M phases of the cell-cycle were downloaded from http://www.cell.com/cms/attachment/2051395126/2059328514/mmc2.xlsx [82]. A set of cell-cycle genes to assess overall proliferation (see below for scoring procedure) was defined as the union of the G1/S and G2/M sets.

\section{Scoring cells using signature gene sets}

To score a specific set of $n$ genes in a given cell, a 'background' gene set was defined to control for differences in sequencing coverage and library complexity between cells [83]. The background gene set was selected to be similar to the genes of interest in terms of expression level. Specifically, the $10 n$ nearest gene neighbors in the 2-D space defined by mean expression and detection frequency across all cells were selected. The signature score for that cell was then defined as the mean expression of the $n$ signature genes in that cell, minus the mean expression of the $10 n$ background genes in that cell.

\section{Testing for shifts in cell proportions in intestinal organoids}

Under several conditions, we observed dramatic changes in the frequency of epithelial cell subtypes (Figure 3). The statistical significance of these shifts was assessed by calculating, for each condition comparison and cell type, the exact hypergeometric probability (without replacement) of the observed change in cell numbers.

Specifically, given that $\boldsymbol{m}$ and $\boldsymbol{n}$ total cells (of all cell types) are sequenced in a treatment and control condition respectively, we test, for a given cell type, whether the 
number of $\boldsymbol{k}$ and $\boldsymbol{q}$ of observed cells of type $\boldsymbol{C}$ in total and treatment condition respectively, significantly deviates from a null model given by the hypergeometric distribution. The probability of observing these values was calculated using the $R$ function 'phyper' from the 'stats' package, using the command:

$$
P=\operatorname{phyper}(\mathrm{q}, \mathrm{k}, \mathrm{m}, \mathrm{n})
$$

and was reported as a hypergeometric $p$-value.

\section{Testing for shifts in cell proportions in vivo}

In the case of in vivo perturbation experiments (Figure 4), we used a regression model to control for any mouse-to-mouse variability amongst our biological replicates. For each cell-type, we model the number of cells detected in each analyzed mouse as a random count variable using a negative binomial distribution. The rate of detection is then modeled by using the natural log of the total number of cells profiled in a given mouse as an offset variable. The condition of each mouse (i.e., knock-out or wild-type) was provided as a covariate. The model was fit using the $\mathrm{R}$ command ' $\mathrm{glm}$ ' from the 'stats' package. The $p$-value for the significance of the effect produced by the knock-out was then assessed using a Wald test.

\section{GO analysis}

GO analysis was performed using the 'goseq' $\mathrm{R}$ package [84], using significantly differentially expressed genes (FDR $<0.05)$ as target genes, and all genes expressed with $\log _{2}(\mathrm{TPM}+1)>3$ in at least 10 cells as background. 


\section{Supplementary Figure legends}

Figure S1. Identification of $\mathrm{LgrF}^{+}$stem cells by single cell RNA-seq, A. Intestinal stem cells (ISCs) identified from scRNA-seq data by unsupervised clustering and posthoc annotation. tSNE visualization of 1,522 single cells (points) profiled by full-length scRNA-seq [81]. Cells are colored by the mean expression (mean $\log _{2}(\mathrm{TPM}+1)$, color bar) of a previously published [85] ISC gene signature (left), the marker gene Lgr5 $\left(\log _{2}(\mathrm{TPM}+1)\right.$, color bar, center left), or by a color code of clusters from $k \mathrm{NN}$-graph clustering (center right), which identifies two clusters (Stem-1 and Stem-2, dark green and cyan, respectively) both of which are positive for the ISC signature genes and express Lgr5. The union of these two clusters (dark green, right) forms a set of 637 ISCs, which were used for further analyses. EP: Enterocyte progenitor, EEC: enteroendocrine cell. B. Quality control. Violin plots show the distributions of reads per cell $(y$-axis, left) and genes detected per cell ( $y$-axis, right) in each IEC-type ( $x$-axis, as defined in (A)). Horizontal bars: median. C. All ISC subsets express a stemness signature. Violin plot showing mean expression $\left(\log _{2}(\mathrm{TPM}+1), y\right.$-axis) of stem cell signature genes [85], in each of the three ISC subsets as well as in the cluster of 201 TA progenitors (Figure S1A) (x-axis). * $p<0.001,{ }^{* *} p<1 \times 10^{-5},{ }^{* * *} p<1 \times 10^{-7}$ (MannWhitney U-test). D,E. Validation of in silico cell type identification using FACS. (D) Proportion (percentage, y-axis) of cells from FACS sorted EpCAM ${ }^{+}$, Lgr5 $^{\text {Low }}$, Lgr5 ${ }^{\text {High }}$, $\mathrm{CD}_{24}{ }^{+}$and $\mathrm{CD} 24^{+} / \mathrm{c}-\mathrm{Kit}^{+}$fractions (color legend) in each of the cell type clusters (bars), identified in the 1,522 IECs sequenced using full-length scRNA-seq (as in (A), Methods). At least $50 \%$ of the cells in each of the ISC subsets are $\operatorname{Lgr} 5^{\text {High }}$, while less 
than $15 \%$ of the cells in any other subset are Lgr5 $^{\text {High }}$. (E) Proportion (percentage, $y$ axis) of cells from each identified cluster (color legend) in each of the FACS fractions (bars). $90.3 \%$ of cells in the $\mathrm{Lgr} 5^{\mathrm{High}}$ fraction are assigned to one of the three ISC states. F. Three ISC subsets are similarly represented along the small intestine. Heatmap shows the fraction (color legend) of cells in each of the detected ISC states (rows) among the ISC isolated from each of three spatial regions (columns), as inferred using a random forest classifier trained on 2,965 ISCs [81], extracted from each of the respective gut regions (Methods). G. Cell type-enriched ligands and receptors. Average relative expression (Z-score of mean $\log _{2}(\mathrm{TPM}+1)$, color bar) of the top 10 receptors (left) and ligands (right, columns) enriched (FDR<0.05, Mann-Whitney U-test) in each cell type (rows). The invariant chain of $\mathrm{MHCII}, \mathrm{Cd74}$, is highlighted in red (left).

Figure S2. Identification and characterization of MHCll-expressing $\mathrm{Lgr5}^{+}$stem cells. A. MHCII signature is largely restricted to ISCs. Violin plot shows the distribution of mean expression levels $\left(\log _{2}(\mathrm{TPM}+1), y\right.$-axis, bar: median) of MHCll genes $(H 2-A b 1$, H2-Aa, Ciita, Cd74, H2-DMa, H2-DMb1) in IEC types (Figure S1A) from the 1,522 IECs profiled by full-length scRNA-seq. EP: Enterocyte progenitor, EEC: enteroendocrine cell. B. Protein-level quantification via FACS of MHCIl expression in IECs from Lgr5GFP mouse. Distribution (left) and mean (bar plot, right) of detected fluorescence corresponding to MHCIl protein expression in populations sorted for GFP-high, GFP-

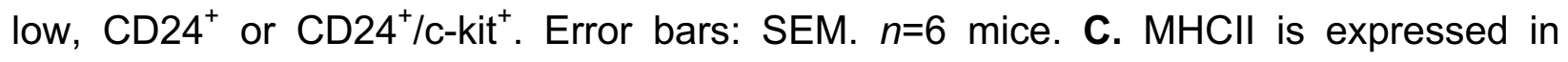
intestinal crypts of wild type mice. IHC images show MHCII expression (I-A/I-E, brown) within crypts of WT mice ( $n=2$ mice). Red arrow, $\mathrm{MHCI}^{+}$cell. Scale bar, $20 \mu \mathrm{m}$. D,E. 
Known quiescence marker $\mathrm{Kdm} 5 b$ identifies post-mitotic cells and low-cycling ISC-I

subset. (D) Scatter plot shows the negative relationship between cell-cycle score $(x-$ axis) and the mean expression of the putative quiescence marker Kdm5b [86-90] $\left(\log _{2}(T P M+1), y\right.$-axis) in each IEC-type (dots) for both proliferating (blue) and postmitotic (red) cells. Trend line shows the random-effects linear model fit to all 1,522 cells. Error bars: SEM. (E) Violin plot of the distribution of expression level of $K d m 5 b$ $\left(\log _{2}(\mathrm{TPM}+1), y\right.$-axis) in ISC-I, ISC-II, and ISC-III clusters $(x$ axis $)\left({ }^{*} p<0.05,{ }^{* *} p<0.005\right.$ Mann-Whitney U test).

Figure S3. ISC states are distinguishable beyond proliferation. A. Distinct signatures of the three ISC subsets. Shown are cumulative distribution functions (CDF) of signature scores (x-axis) in the cells from each of the three subsets of ISCs (colored curves) of a published ISC signature from bulk data [85] (left), or signatures of 25 genes defined by differential expression testing in each subset (Methods). B. Two ISC subsets found in $\mathrm{GO}$ are varying by MHCII expression. Top left: Scatter plot of the G1/S (x-axis) and G2/M (y-axis) signature scores for 637 Lgr5 ${ }^{+}$ISCs (points). A subset of 183 $\mathrm{Lgr5}^{+}$ISCs that are likely in G0 is marked in purple. Other panels: PCA of these 183 ISCs, where cells (dots; density marked by contours) are colored by the expression $\left(\log _{2}(\mathrm{TPM}+1)\right.$, color bar) of $\mathrm{MHCll}$ (top right panel) or by three ISC-I markers (bottom panels). Two subsets of cells are separated by $\mathrm{PC}-1$ : one is $\mathrm{MHCI}^{\text {low }}$ and positive for ISC-I markers and the other is $\mathrm{MHCII}^{\text {high }}$. C. MHCII ${ }^{\text {high }}$ ISCs sorted by FACS express higher levels of MHCII mRNA. Violin plot shows the distribution of the mean expression level $\left(y\right.$-axis, $\left(\log _{2}(\mathrm{TPM}+1)\right)$ of the MHCIl gene signature (Methods) in each cell (dot) in 
$\mathrm{Lgr}^{\text {high }}$ cells sorted on $\mathrm{MHCI}{ }^{\text {low }}$ and $\mathrm{MHCII}^{\text {high }}\left(x\right.$-axis) $\left(^{* * *} p<0.0005\right.$, Mann-Whitney $U$ test).

Figure S4. Changes in immune cells, IEC cell-type composition and ISC gene expression in response to pathogen infection in vivo. A. $\mathrm{CD}^{+} \mathrm{T}$ cells interact with stem cells in vivo. Two-photon microscopy image of the small intestine from Lgr5-GFP (green) knock-in mouse engrafted with $\mathrm{RFP}^{+} \mathrm{CD} 4^{+} \mathrm{T}$ cells (red). $\mathrm{CD} 4^{+} \mathrm{T}$ cells are visible

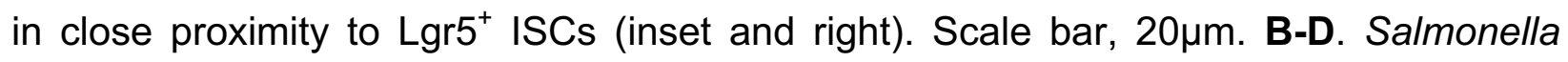
enterica infection induces Th1 polarization in the gut. (B) Changes in immune cell proportions. Stacked bar plots show the percentage ( $y$-axis) of different immune cell subsets (color legend), as determined by scRNA-seq of $5,122 \mathrm{CD}^{+} 5^{+}$cells from the lamina propria of control and Salmonella-infected mice. (C) Bar plot shows the significance of the enrichment $\left(-\log _{10}(p\right.$-value $), y$-axis, hypergeometric test) of marker genes for different $T$ helper subsets $(x$-axis) among the genes induced (FDR $<0.05$, likelihood-ratio test) in T cells from Salmonella infected vs. control mice. Dashed line: $p=0.05$. (D) Plot shows differential expression ( $x$-axis) for each gene ( $y$-axis) across 824 T cells from Salmonella-infected mice $(n=4)$ and 543 T cells from control mice $(n=5)$. Bar indicates Bayesian bootstrap [91] estimates of $\log _{2}$ (fold-change), and hinges and whiskers indicate $25 \%$ and $95 \%$ confidence intervals, respectively. Th1 cell markers are labeled in green. Dashed line: no differential expression. E,F. Changes in fractions of tuft and Paneth cells within the intestinal epithelium after infection. Bar plots show the frequencies of tuft $(\mathbf{E})$ and Paneth $(\mathbf{F})$ cells ( $y$-axis), as determined by unsupervised clustering of droplet-based scRNA-seq data in mice under different conditions ( $x$-axis) 
[81], $n=2$ and 4 mice (points) per group, in (E) and (F), respectively. ${ }^{*}$ FDR $<10^{-5}$; ** FDR $<10^{-10}$, likelihood-ratio test, Methods. G. Fraction of ISCs within the intestinal epithelium after infection. Cell-type frequencies ( $y$-axis) determined by unsupervised clustering of droplet-based scRNA-seq data in each infection model and control mice ( $x$ axis, $n=2$ mice (points) per infection group, $n=4$ for control; ${ }^{* *}$ FDR $<10^{-10}$ likelihood-ratio test, Methods). H. Reduced stemness scores in ISCs during pathogenic infection in vivo. Violin plot shows the distribution of the bulk stemness signature score (y-axis, Methods) of 1,857 ISCs identified by clustering (Methods) of 9,842 cells sequenced using droplet-based scRNA-seq from Salmonella enterica- or $H$. polygyrus-treated mice and controls [81] (x-axis). ${ }^{*} p<0.01,{ }^{* *} p<10^{-5}$ (Mann-Whitney U-test). I. Pathogenic infection reduces the expression of ISC marker genes. Heatmap shows the mean expression (column-wise Z-score of mean $\log _{2}(\mathrm{TPM}+1)$ values, color bar) of all of the known ISC marker genes [85] (columns) that are differentially expressed (FDR<0.05) by 1,857 ISCs, as determined by unsupervised clustering from a total of 9,842 cells profiled by droplet-based scRNA-seq, in control and pathogen-infected mice (rows). J. Shifts in the three ISC subsets under infection in vivo. Heatmap shows the significance of changes of expression scores $\left(\log _{10}(p\right.$-value), Mann-Whitney U-test, color bar, of enrichment (red) and depletion (blue)) within 1,857 ISCs. ISCs in each condition were scored for expression of each ISC subset gene signature (columns, sets and signatures as in Figure 2), and the distribution of scores was compared to that in ISCs from control mice (top three rows). Bottom row: comparison between control mice on day 3 and 10 of the $H$. polygyrus infection course. 
Figure S5. Characterization of intestinal organoids co-cultured with T helper (Th) cells or treated with their key cytokines. A. Intestinal organoid co-cultured with $\mathrm{T}$ cells. Images of organoid and $\mathrm{T}_{\text {reg }}$ co-cultures at $\times 4$ magnification. Lines mark $\mathrm{T}_{\text {regs }}$ and organoids. B,C. Validation of in vitro-polarized Th cell populations. FACS plots for each of the four subsets of Th cells. Top panels: quantification of the viability dye 7-AAD $(y-$ axis) and the Th cell marker CD4 (x-axis). Bottom panels: quantification of relevant marker proteins for each Th subset ( $x$ - and $y$-axes, bottom panels). (C). Heatmap shows the mean relative expression (row-wise Z-score of mean $\log _{2}(T P M+1)$ values, color bar) of canonical marker genes (rows) in the cells from each in vitro-differentiated Th cell subset (columns), identified by droplet-based scRNA-seq of co-cultures (Methods). D. IEC type identification within intestinal organoid cultures. tSNE embeddings of 17,755 single IECs (individual points) isolated from control, cytokine-treated and Th cell cocultured intestinal organoids and sequenced using droplet-based scRNA-seq. Most cells were merged into a single dataset to maximize the statistical power of clustering (Methods). Top left panel: cells colored by cluster assignment from unsupervised $k N N-$ graph clustering (Methods). Top middle panel: cells colored by post-hoc annotations using cell-type signatures derived from in vivo scRNA-seq data (clusters that expressed high levels of the same signatures were merged to a final set of seven clusters). All other panels: cells colored by the mean expression (color bar, $\log _{2}(T P M+1)$ ) of the noted cell-type specific signatures. E. Organoid-derived secretory IECs co-express markers for goblet and Paneth cells. Scatter plots show the expression levels (top three rows, $\log _{2}(\mathrm{TPM}+1)$ ) of canonical markers for goblet cells (Spink4, Agr2, Tff3, y-axis) and for Paneth cells (Defa24, ItIn1, Lyz1, x-axis) or signature scores for goblet and Paneth 
cells (bottom row, 50 genes), for in vivo goblet cells (left), cells in the Paneth-goblet cluster from control organoids (middle) and in vivo Paneth cells (right). F. Reanalysis of published scRNA-seq data confirms Paneth and goblet cell marker co-expression in organoids. tSNE embeddings of $161 \mathrm{Reg} 4^{+}$cells sorted from intestinal organoid cultures in an independent study [70]. Top left: Cell types were identified using the RacelD clustering algorithm, as in the original publication [70] (colored and numbered nodes). Remaining plots: cells are colored by the expression ( $\log _{2}$ (normalized transcript count), color bars) of canonical markers of Paneth and goblet cells. A group of Paneth-gobletlike cells is clearly observed, where individual cells are double-positive for markers of both cell types. G. Induction of MHCII expression in organoids co-cultured with Th1 cells. Violin plot shows the distribution of mean expression levels $\left(\log _{2}(T P M+1), y\right.$-axis, bar denotes the median value) of six MHCll genes (H2-Ab1, H2-Aa, Ciita, Cd74, H2DMa, H2-DMb1) in IECs profiled by droplet-based scRNA-seq from control organoids and those co-cultured with each subset of Th cells (total of 6,234 cells, x-axis). ${ }^{* * *} p<$ $10^{-10}$, (Mann-Whitney U-test).

Figure S6. Changes in cell proportions and ISC expression programs in organoids co-cultured with Th subsets or their signature cytokines. A-C. Changes in proportion of cells expressing stem cell marker genes after co-culture with $\mathrm{iT}_{\text {regs }}$ or treatment with IL-10. A,B. Scatter plots compare the fraction of cells showing non-zero expression ( $\alpha, y$-axis) of each gene (dot) in organoids $(\mathbf{A})$ co-cultured with $\mathrm{iT}_{\text {reg-cells or }}$ (B) treated with IL-10, compared to the fraction in matching control organoids ( $\alpha, x-$ axis). All genes up-regulated $(F D R<0.05)$ are shown, sized relative to their significance 
$\left(-\log _{10}(\right.$ FDR $)$, legend top left). Several key ISC marker genes (Methods) are labeled. The diagonal line indicates no change relative to the control organoid. (C) Heatmap shows the fraction of cells with non-zero expression ( $\alpha$, color bar) of 10 selected stem cell marker genes (columns) within organoids co-cultured with $\mathrm{T}_{\text {regs }}$ or Th1 cells (top, rows) or treated with IL-13 or IL-10 cytokines (bottom, rows). All genes are expressed at a larger proportion of cells (FDR < 0.05, Mann-Whitney U-test) in organoids co-cultured with $\mathrm{iT}_{\text {reg }}$ cells (top) or treated with IL-10 (bottom), compared to control organoids. D-F. Th1 co-culture up-regulates Paneth cell-related gene expression in organoids. (D) Heatmap of the significance of change of the Paneth cell signature score in 'Panethgoblet' cells between different Th co-cultures (rows) and control organoids $\left(-\log _{10}(p\right.$ value), Mann-Whitney U-test, color bar, of enrichment (red) and depletion (blue). (E) Density histograms of the distribution of Paneth cell signature scores in 'Paneth-goblet' cells in organoids co-cultured with either Th1 (red, left) or Th2 (red, right) cells compared to their matching control organoids (blue). Dashed lines denote the mean score. * $p<0.05,{ }^{* *} p<10^{-5}$ (Mann-Whitney U-test). (F) Box plots of the distribution of expression levels $\left(\log _{2}(\mathrm{TPM}+1), y\right.$-axis) of canonical Paneth cell markers Defa24 (left) and Lyz1 (right) in the IEC-type cluster (x-axis) from organoids co-cultured with Th1 cells (right part of each panel) compared to control organoids (left part). ${ }^{* *} p<10^{-5}$ (Mann-Whitney U-test).

Figure S7. Validation of MHCIl knockout in intestinal epithelial cells. A,B. Validation of epithelial-specific MHCII knockout by FACS quantification of MHCIIexpressing IECs in small intestine $(\mathbf{A})$ and mesenteric lymph node (B). Scatter plots 
(left) and bar plots (right) show the fraction of $\mathrm{EpCAM}^{+} / \mathrm{MHCll}^{+}(\mathbf{A})$ or $\mathrm{CD} 11 \mathrm{~b}^{+} / \mathrm{MHCll}^{+}$ (B) cells in $\mathrm{MHCII}^{\mathrm{fl} / \mathrm{fl}}$ and $\mathrm{MHCll}$ knockout mice $\left(\mathrm{MHCll}^{\Delta \text { gut }}\right)$. (A) $n=5$ mice, ${ }^{*} p<0.05$. (B) $n=2$ mice, NS: not statistically significant.

Figure S8. Impact of MHCII knockout in gut epithelial cells on the ISC pool. A. Increased proportion of $\mathrm{LgrF}^{+}$cells in $\mathrm{MHClI}^{\Delta \text { gut }}$ mice. Bar plot shows the fraction of cells (y-axis) in which the transcript for Lgr5 is detected, amongst the 1,559 cells profiled from $\mathrm{MHClI}^{\Delta \text { gut }}$ mice $(n=5)$ and 1,617 cells profiled from matched $\mathrm{MHCII}^{\mathrm{fl} / \mathrm{ll}}$ controls $(n=5)$. Error bars: SEM, ${ }^{*} p<0.05$, likelihood-ratio test. B. scRNA-seq of IECs from $\mathrm{MHCII}^{\Delta \mathrm{gut}}$ and matched controls. tSNE embedding of 3,176 cells colored by their genotype (color legend, left), assignment to cell types by unsupervised clustering (middle, Methods) and stemness score (right; score as in Figure S1A). C,D. Expansion of ISCs following $\mathrm{KO}$ of $\mathrm{MHCll}$ specifically in gut epithelial cells. Bar plot (C) and volcano plot (D) based on all 1,559 cells in $\mathrm{MHClI}^{\Delta \text { gut }}$ mice $(n=5)$ vs. 1,617 cells from matched $\mathrm{MHCII} / \mathrm{fl}$ controls (n=5). Green dots in (D): up-regulated ISC genes, red dots: down-regulated ISC genes (FDR<0.05, likelihood-ratio test), grey dots: non-DE genes. E. Higher stemness signatures in ISCs from $\mathrm{MHCI}^{\Delta \text { gut }}$ mice. Violin plots of the distribution of the signature scores for the cell-cycle (as in Figure 2A), MHCII genes and stemness [85] (as in Figure 1C), in ISCs from $\mathrm{MHCI}^{\mathrm{fl} / f \mathrm{l}}$ mice $(n=5$, grey) and from $\mathrm{MHCII}^{\Delta \text { gut }}$ mice ( $n=5$, white), when including either all 381 ISCs (top left), only the 173 ISCs that still have detectable mRNA for H2-Ab1 (bottom left), or only the 208 ISCs that are a confirmed $\mathrm{KO}$ : do not have detectable mRNA for $H 2-A b 1$ (bottom right). 
Expression of $H 2-A b 1$ mRNA is also shown in these two groups (top right). Small dots: individual cells; squares: mean per mouse; ${ }^{*} p<0.05$, likelihood-ratio test.

Figure S9. An expanded ISC pool in T cell-depleted mouse models. Volcano plots $(\mathbf{A}, \mathbf{C})$ show mean $\log _{2}$ fold-change (x-axis) and significance (- $\log _{10}(F D R)$, Methods) of differential expression between 7,216 cells from WT mice $(n=6), 2,967$ cells from nude mice $(\mathbf{A}, n=2$ mice) or 9,488 cells from TCR $\beta-K O$ mice ( $\mathbf{C}, n=2$ mice). Green dots: upregulated ISC genes, red dots: down-regulated ISC genes (FDR<0.05, likelihood-ratio test), grey dots: non-DE genes. Bar plots $(\mathbf{B}, \mathbf{D})$ show frequency ( $y$-axis) of each major IEC-type ( $x$-axis), as determined by unsupervised clustering (Methods), in cells from WT (grey) vs. nude (white, B) or TCR - KO mice (white, D). Dots correspond to individual mice. Error bars are SEM. ( ${ }^{*}$ FDR $<0.05$, ** FDR $<0.005$, *** FDR $<10^{-5}$, likelihood-ratio test). E,F. ISC expansion in two T cell-depleted mouse models. (E) Bar plot shows the fraction of epithelial cells which are stem cells ( $y$-axis), as determined by unsupervised clustering of scRNA-seq profiles, from WT (7,216 cells, $n=6)$, nude $(2,967$ cells, $n=2)$, and TCR ${ }_{-} \mathrm{KO}$ mice $(9,488$ cells, $n=2)$. Dots correspond to individual mice. Error bars are SEM. ( ${ }^{*} p<0.05,{ }^{* *} p<10^{-3},{ }^{* * *} p<10^{-5}$, likelihood-ratio test, Methods). (F) smFISH of $L g r 5^{+}$cells in the crypt. Left: Lgr5 expression (red) in intestinal crypts of wild

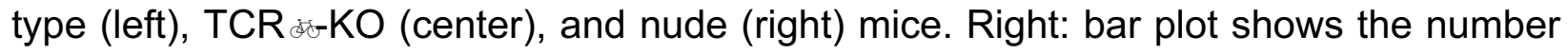
of Lgr5 molecules detected per crypt ( $y$-axis) in each of the three models (x-axis). $n=2$ mice and 8 fields per group. Error bars: $\mathrm{SD}\left({ }^{*} p<0.05,{ }^{* *} p<0.005, t\right.$-test, Methods). 
Figure S10. Impact of $\mathrm{T}_{\text {reg }}$ depletion on ISC pool in vivo. A-E. Reduction of the ISC pool following $\mathrm{T}_{\text {reg }}$ depletion in Foxp3-DTR mice. A. Representative FACS plot (left) and quantified mean proportion (bar plot, right) of $\mathrm{TCR}^{+} \mathrm{Foxp} 3^{+} \mathrm{T}_{\text {regs }}$ out of all $\mathrm{CD} 4^{+} \mathrm{TCR} \beta^{+}$ cells in the small intestine of WT and Foxp3-DTR mice ( $y$-axis) after 7 days of DT treatment. Dots: individual mice. Error bars: SEM ( $n=3$ mice, ${ }^{* * *} p<0.0005 t$-test). B. IHC images of FFPE sections stained for H\&E (left), cleaved Caspase-3 (brown, middle) and Ki67 (brown, right) in WT (top row) and Foxp3-DTR mice (bottom row) after 7 days of DT treatment. Inset is $3 \times$ magnification showing $T_{\text {reg }}$ depletion results in proliferation at the bottom of the crypts, where stem cells reside, with no signs of apoptosis. Scale bar, $50 \mu \mathrm{m}$. C. Bar plot of the frequency of cells in which Lgr5 mRNA is detected (y-axis) in WT and Foxp3-DTR mice both treated with DT. (Error bars: SEM, ${ }^{* *} p<0.005$ likelihoodratio test). D. Volcano plot shows mean $\log _{2}$ (fold-change, $x$-axis) and significance ($\log _{10}(F D R)$, Methods) of differential expression between 815 cells from Foxp3-DTR mice $(n=4)$ vs. 2,572 cells from matched WT controls $(n=5)$ both treated with DT. Green dots: upregulated ISC genes, Red dot: downregulated ISC genes (FDR<0.05, likelihood-ratio test, grey dots: non-DE genes. E. Bar plot shows frequency (y-axis) of IEC-types, as determined by unsupervised clustering (Methods), in 815 cells from Foxp3-DTR mice ( $n=4$, white) vs. 2,572 cells from matched WT controls ( $n=5$, grey) both treated with DT. Individual mice marked by points. Error bars: SEM. $\left(^{* *}\right.$ FDR $<0.005,{ }^{* * *}$ FDR $<10^{-5}$ likelihood-ratio test). F. Expansion of tuft cells following $T_{\text {reg }}$ depletion in Foxp3-DTR mice. IFA image of $\mathrm{DCLK}^{+}$(red) tuft cells in the epithelia of wild type (WT, left) and Foxp3-DTR mice (right) both treated with DT. Scale bar, 50um. 


\section{References}

58. Shalek, A.K., Satija, R., Shuga, J., Trombetta, J.J., Gennert, D., Lu, D., Chen, P., Gertner, R.S., Gaublomme, J.T., Yosef, N., et al. (2014). Single-cell RNA-seq reveals dynamic paracrine control of cellular variation [Supplementrary Information]. Nature, 1-28.

59. Su, L., Su, C.W., Qi, Y., Yang, G., Zhang, M., Cherayil, B.J., Zhang, X., and Shi, H.N. (2014). Coinfection with an intestinal helminth impairs host innate immunity against Salmonella enterica serovar Typhimurium and exacerbates intestinal inflammation in mice. Infect Immun 82, 3855-3866.

60. Picelli, S., Faridani, O.R., Bjorklund, A.K., Winberg, G., Sagasser, S., and Sandberg, R. (2014). Full-length RNA-seq from single cells using Smart-seq2. Nat Protoc 9, 171181.

61. Habib, N., Li, Y., Heidenreich, M., Swiech, L., Avraham-Davidi, I., Trombetta, J.J., Hession, C., Zhang, F., and Regev, A. (2016). Div-Seq: Single-nucleus RNA-Seq reveals dynamics of rare adult newborn neurons. Science 353, 925-928.

62. Biton, M., Levin, A., Slyper, M., Alkalay, I., Horwitz, E., Mor, H., Kredo-Russo, S., AvnitSagi, T., Cojocaru, G., Zreik, F., et al. (2011). Epithelial microRNAs regulate gut mucosal immunity via epithelium-T cell crosstalk. Nat Immunol 12, 239-246.

63. Schneider, C.A., Rasband, W.S., and Eliceiri, K.W. (2012). NIH Image to ImageJ: 25 years of image analysis. Nat Methods 9, 671-675.

64. Matsumoto, T., Mochizuki, W., Nibe, Y., Akiyama, S., Matsumoto, Y., Nozaki, K., Fukuda, M., Hayashi, A., Mizutani, T., Oshima, S., et al. (2016). Retinol Promotes In Vitro Growth of Proximal Colon Organoids through a Retinoic Acid-Independent Mechanism. PLoS One 11, e0162049.

65. Iwata, M., Hirakiyama, A., Eshima, Y., Kagechika, H., Kato, C., and Song, S.Y. (2004). Retinoic acid imprints gut-homing specificity on T cells. Immunity 21, 527-538.

66. Esplugues, E., Huber, S., Gagliani, N., Hauser, A.E., Town, T., Wan, Y.Y., O'Connor, W., Jr., Rongvaux, A., Van Rooijen, N., Haberman, A.M., et al. (2011). Control of TH17 cells occurs in the small intestine. Nature 475, 514-518.

67. Langmead, B., Trapnell, C., Pop, M., and Salzberg, S.L. (2009). Ultrafast and memoryefficient alignment of short DNA sequences to the human genome. Genome Biology.

68. Beuling, E., Baffour-Awuah, N.Y., Stapleton, K.A., Aronson, B.E., Noah, T.K., Shroyer, N.F., Duncan, S.A., Fleet, J.C., and Krasinski, S.D. (2011). GATA factors regulate proliferation, differentiation, and gene expression in small intestine of mature mice. Gastroenterology 140, 1219-1229 e1211-1212.

69. Brennecke, P., Anders, S., Kim, J.K., Kołodziejczyk, A.A., Zhang, X., Proserpio, V., Baying, B., Benes, V., Teichmann, S.A., Marioni, J.C., et al. (2013). Accounting for technical noise in single-cell RNA-seq experiments. Nature Methods 10, 1093-1095.

70. Grün, D., Lyubimova, A., Kester, L., Wiebrands, K., Basak, O., Sasaki, N., Clevers, H., and van Oudenaarden, A. (2015). Single-cell messenger RNA sequencing reveals rare intestinal cell types. Nature, 1-23.

71. Johnson, W.E., Li, C., and Rabinovic, A. (2007). Adjusting batch effects in microarray expression data using empirical Bayes methods. Biostatistics (Oxford, England) 8, 118-127. 
72. Leek, J.T., Johnson, W.E., Parker, H.S., Jaffe, A.E., and Storey, J.D. (2012). The sva package for removing batch effects and other unwanted variation in highthroughput experiments. Bioinformatics 28, 882-883.

73. Erichson N. Benjamin, V.S., Brunton Steven L., Kutz J. Nathan (2016). Randomized Matrix Decompositions using R. arXiv preprint

74. Buja, A., and Eyuboglu, N. (1992). Remarks on Parallel Analysis. Multivariate Behavioral Research 27, 509-540.

75. van der Maaten, L. (2014). Accelerating t-SNE using Tree-Based Algorithms. The Journal of Machine Learning Research 15, 3221-3245.

76. van der Maaten, L., and Hinton, G. (2008). Visualizing Data using t-SNE. The Journal of Machine Learning Research 9, 2579-2605.

77. Rosvall, M., and Bergstrom, C.T. (2008). Maps of random walks on complex networks reveal community structure. Proceedings of the National Academy of Sciences 105, 1118-1123.

78. Levine, J.H., Simonds, E.F., Bendall, S.C., Davis, K.L., Amir, E.-a.D., Tadmor, M.D., Litvin, O., Fienberg, H.G., Jager, A., Zunder, E.R., et al. (2015). Data-Driven Phenotypic Dissection of AML Reveals Progenitor-like Cells that Correlate with Prognosis. Cell, 1-15.

79. Shekhar, K., Lapan, S.W., Whitney, I.E., Tran, N.M., Macosko, E.Z., Kowalczyk, M., Adiconis, X., Levin, J.Z., Nemesh, J., Goldman, M., et al. (2016). Comprehensive Classification of Retinal Bipolar Neurons by Single-Cell Transcriptomics. Cell 166, 1308-1323.e1330.

80. Sato, T., Vries, R.G., Snippert, H.J., van de Wetering, M., Barker, N., Stange, D.E., van Es, J.H., Abo, A., Kujala, P., Peters, P.J., et al. (2009). Single Lgr5 stem cells build cryptvillus structures in vitro without a mesenchymal niche. Nature 459, 262-265.

81. Haber, A.L., Biton, M., Rogel, N., Herbst, R.H., Shekhar, K., Smillie, C., Burgin, G., Delorey, T.M., Howitt, M.R., Katz, Y., et al. (2017). A single-cell survey of the small intestinal epithelium. Nature doi:10.1038/nature24489.

82. Macosko, E.Z., Basu, A., Satija, R., Nemesh, J., Shekhar, K., Goldman, M., Tirosh, I., Bialas, A.R., Kamitaki, N., Martersteck, E.M., et al. (2015). Highly Parallel Genomewide Expression Profiling of Individual Cells Using Nanoliter Droplets. Cell 161, 1202-1214.

83. Kowalczyk, M.S., Tirosh, I., Heckl, D., Rao, T.N., Dixit, A., Haas, B.J., Schneider, R.K., Wagers, A.J., Ebert, B.L., and Regev, A. (2015). Single-cell RNA-seq reveals changes in cell cycle and differentiation programs upon aging of hematopoietic stem cells. Genome Research 25, 1860-1872.

84. Young, M.D., Wakefield, M.J., Smyth, G.K., and Oshlack, A. (2010). Gene ontology analysis for RNA-seq: accounting for selection bias. Genome Biology 11.

85. Muñoz, J., Stange, D.E., Schepers, A.G., van de Wetering, M., Koo, B.-K., Itzkovitz, S., Volckmann, R., Kung, K.S., Koster, J., Radulescu, S., et al. (2012). The Lgr5 intestinal stem cell signature: robust expression of proposed quiescent \&apos;+4\&apos; cell markers. The EMBO Journal 31, 3079-3091.

86. Patel, A.P., Tirosh, I., Trombetta, J.J., Shalek, A.K., Gillespie, S.M., Wakimoto, H., Cahill, D.P., Nahed, B.V., Curry, W.T., Martuza, R.L., et al. (2014). Single-cell RNA-seq highlights intratumoral heterogeneity in primary glioblastoma. Science 344, 13961401. 
87. Hayami, S., Yoshimatsu, M., Veerakumarasivam, A., Unoki, M., Iwai, Y., Tsunoda, T., Field, H.I., Kelly, J.D., Neal, D.E., Yamaue, H., et al. (2010). Overexpression of the JmjC histone demethylase KDM5B in human carcinogenesis: involvement in the proliferation of cancer cells through the E2F/RB pathway. Molecular Cancer 9, 5914.

88. Roesch, A., Fukunaga-Kalabis, M., Schmidt, E.C., Zabierowski, S.E., Brafford, P.A., Vultur, A., Basu, D., Gimotty, P., Vogt, T., and Herlyn, M. (2010). A Temporarily Distinct Subpopulation of Slow-Cycling Melanoma Cells Is Required for Continuous Tumor Growth. Cell 141, 583-594.

89. Oki, T., Nishimura, K., Kitaura, J., Togami, K., Maehara, A., Izawa, K., Sakaue-Sawano, A., Niida, A., Miyano, S., Aburatani, H., et al. (2014). A novel cell-cycle-indicator, mVenus-p27K-, identifies quiescent cells and visualizes G0-G1 transition. Scientific Reports 4, 1-10.

90. Tirosh, I., Izar, B., Prakadan, S.M., Wadsworth, M.H., 2nd, Treacy, D., Trombetta, J.J., Rotem, A., Rodman, C., Lian, C., Murphy, G., et al. (2016). Dissecting the multicellular ecosystem of metastatic melanoma by single-cell RNA-seq. Science 352, 189-196.

91. Rubin, D.B. (1981). The Bayesian bootstrap. The Annals of Statistics 9, 130-134. 


\section{Supplementary Figure 1}

A Bulk stem cell signature

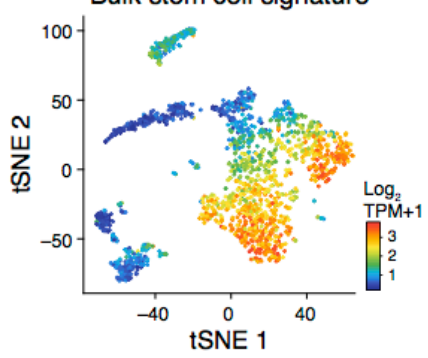

B

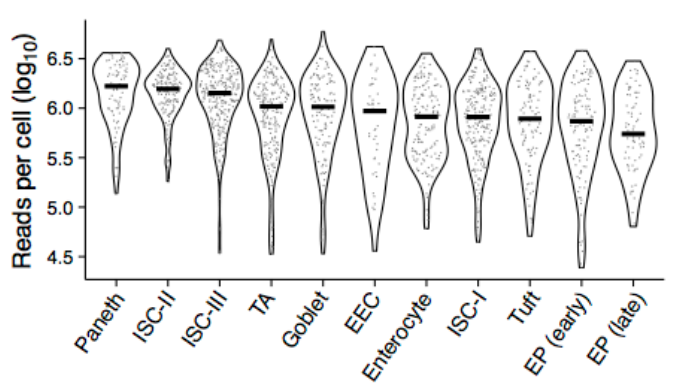

D

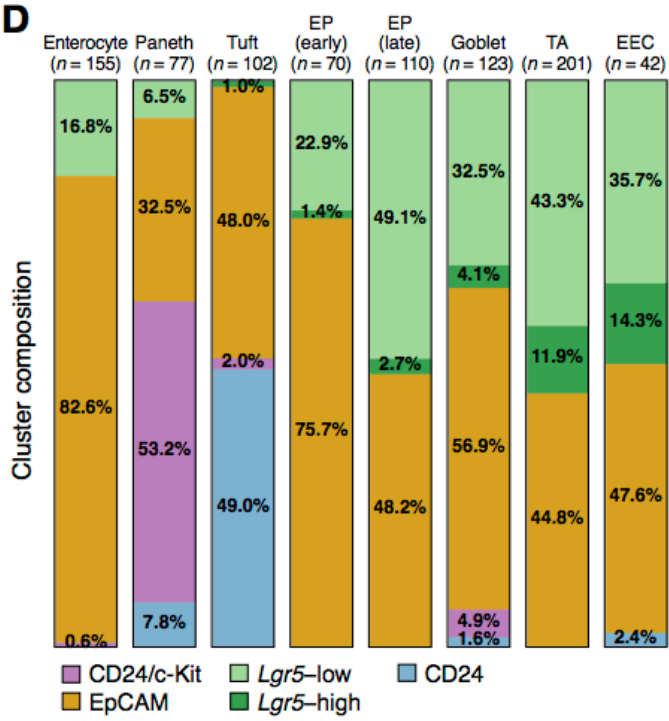

Lgr5

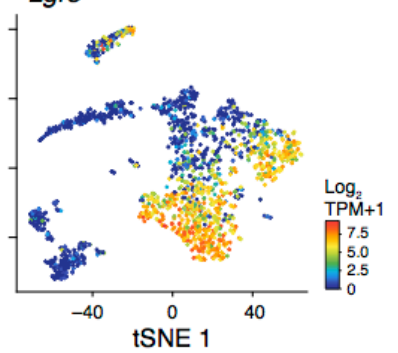

KNN-graph clusters

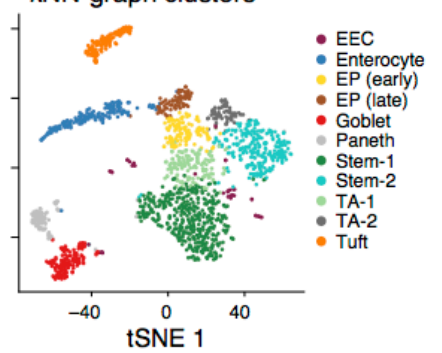

Merged clusters

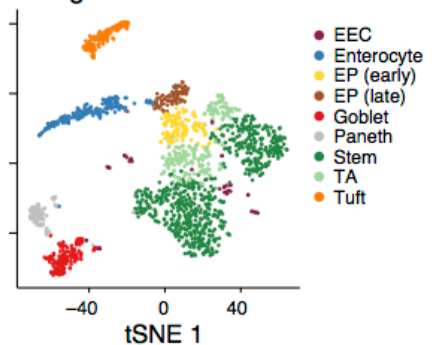

C

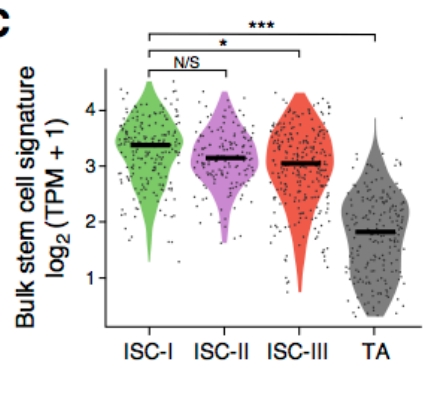

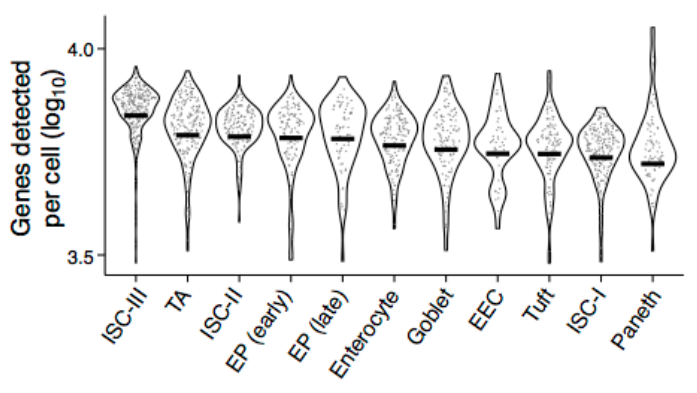

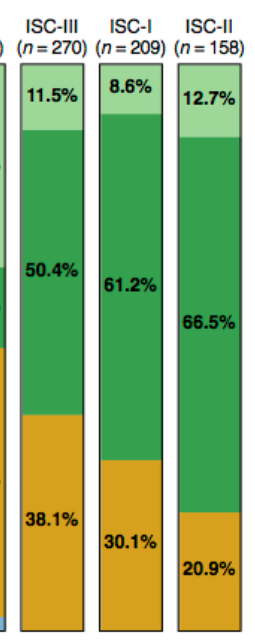

E

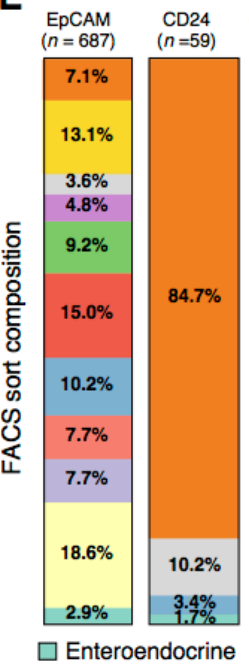

$\square$ Enteroendocrin

$\square$ Enterocyte

$\square$ EP (early)
$\mathbf{F}$

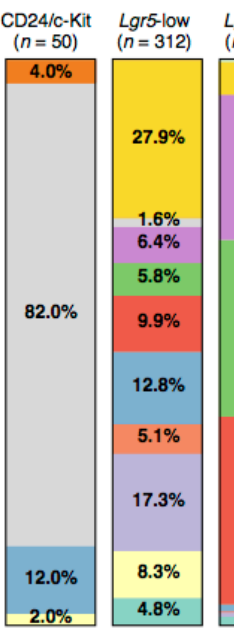

Lgr5-high
$(n=409)$ $0.2 \%$

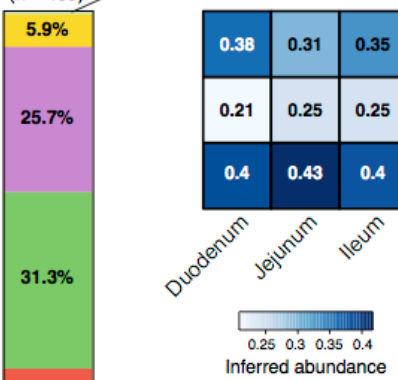

\section{G Receptors}

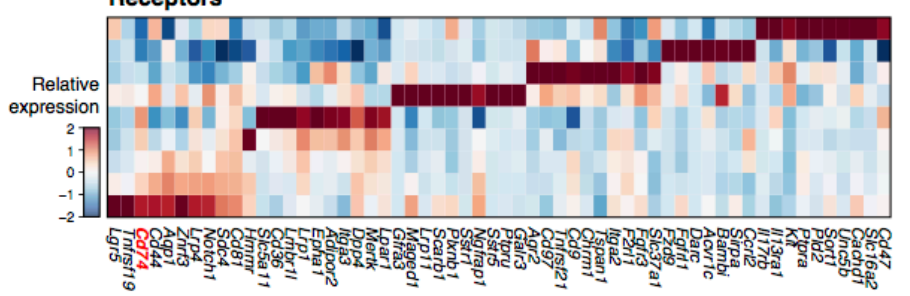

Ligands

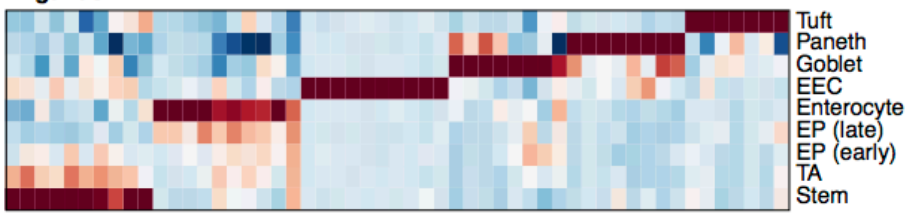

ISC-I ISC-II ISC-III 


\section{Supplementary Figure 2}

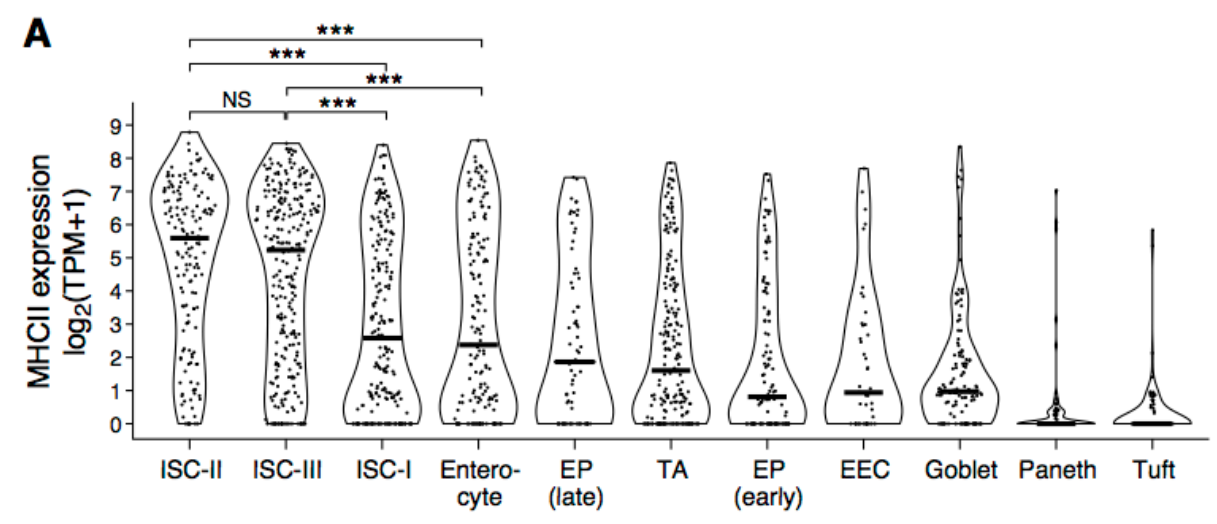

B
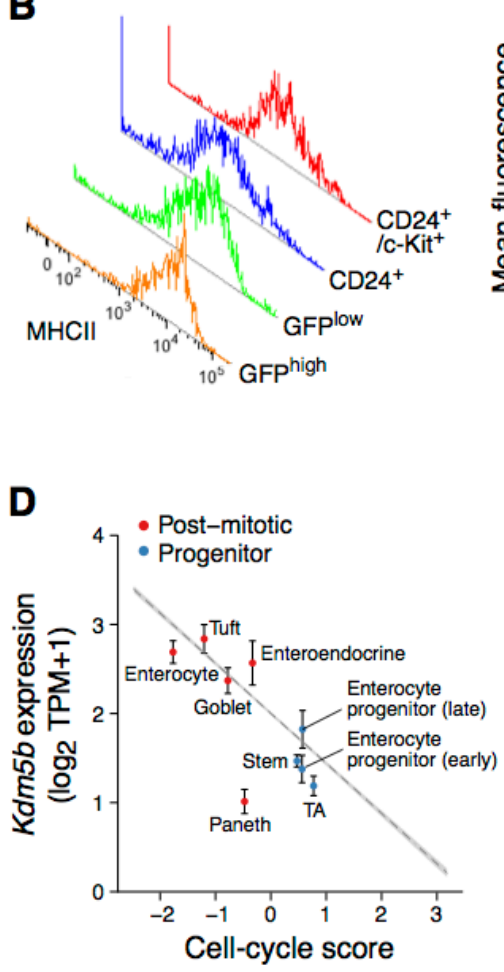

C
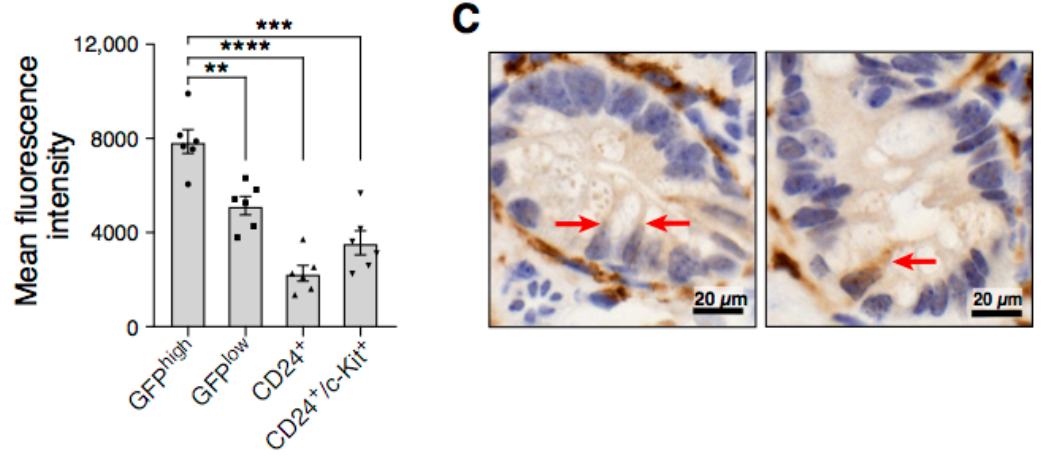

E

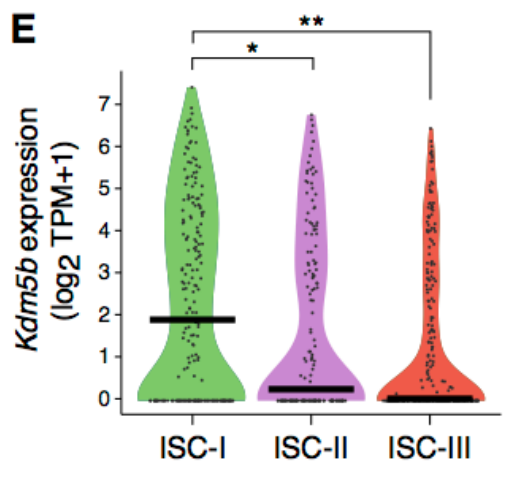




\section{Supplementary Figure 3}

A
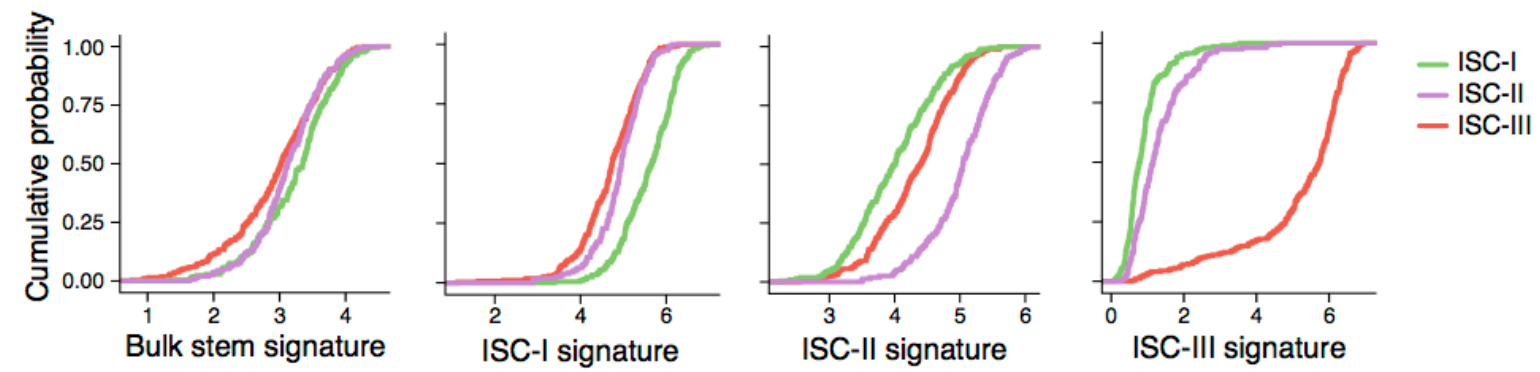

B
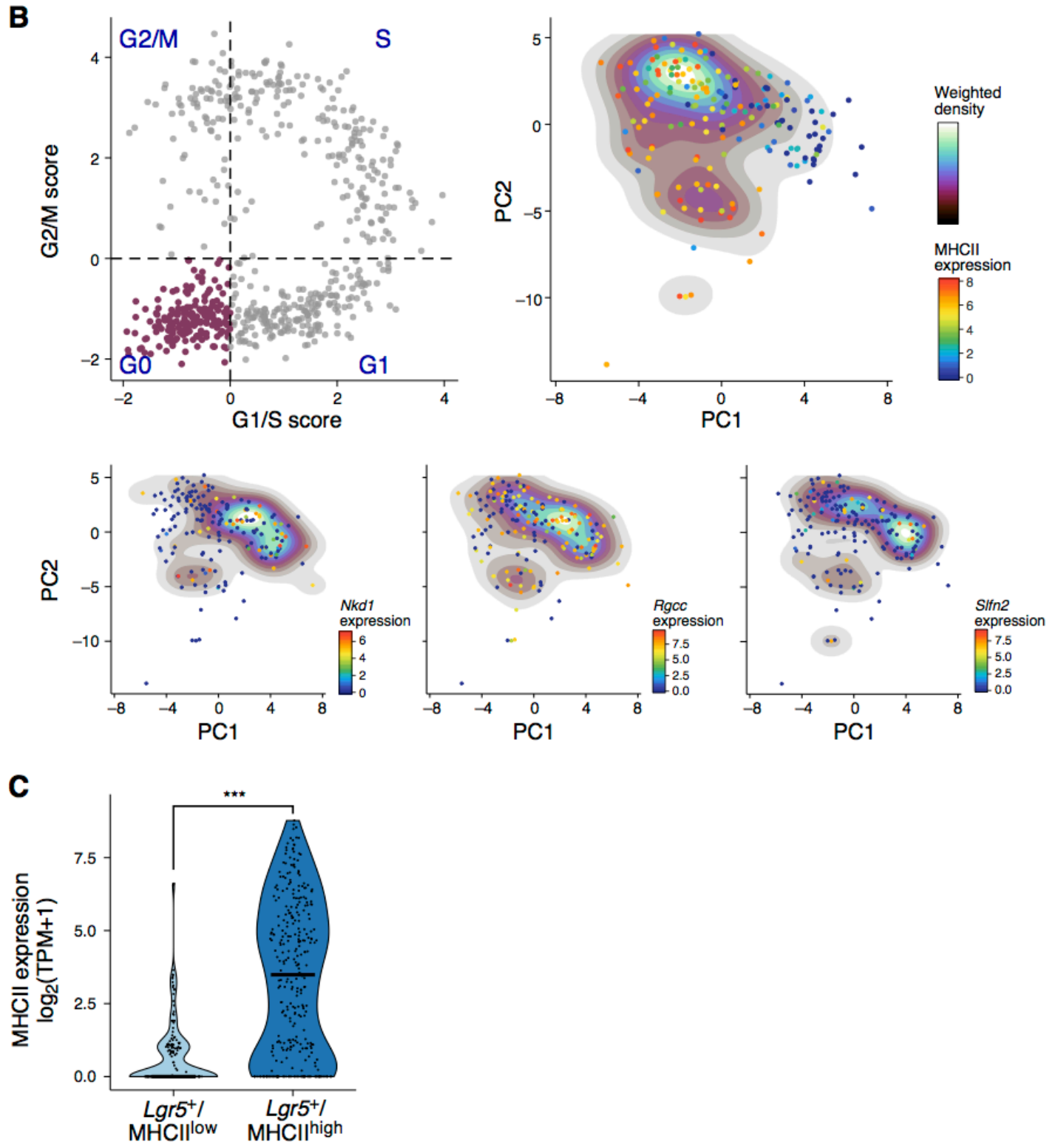


\section{Supplementary Figure 4}

\section{A}

\section{- Lgr5 GFP stem cells • ACTB RFP CD4+ T cells}
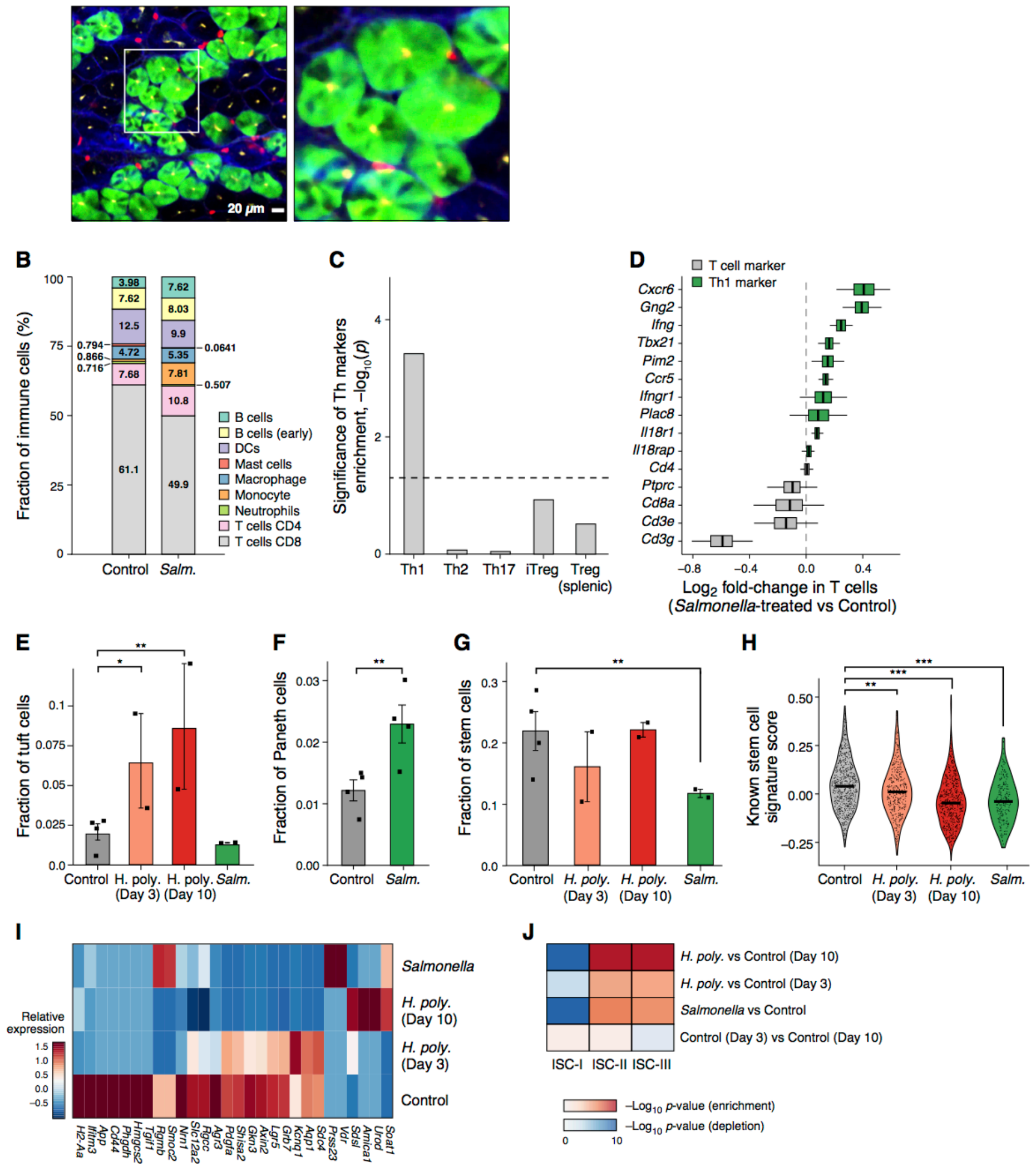


\section{Supplementary Figure 5}

A

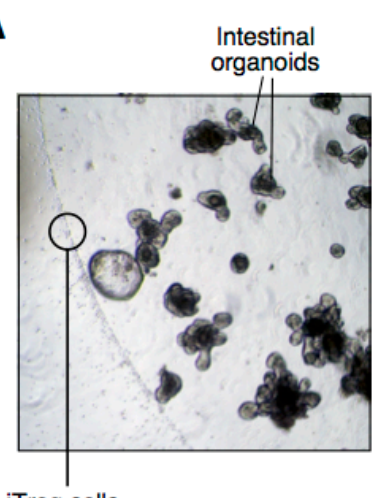

iTreg cells
B
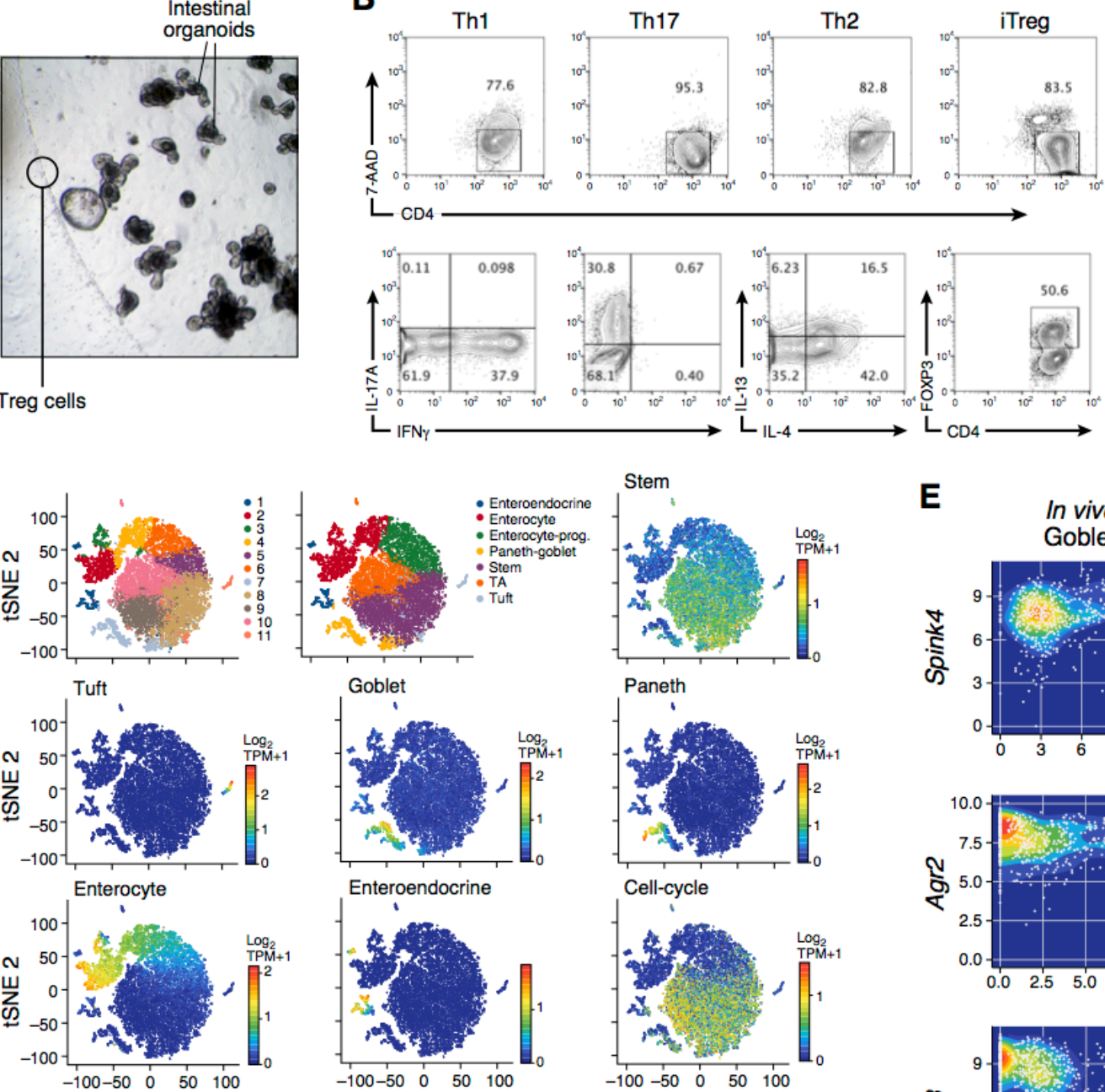

tSNE 1

$\mathbf{F}$
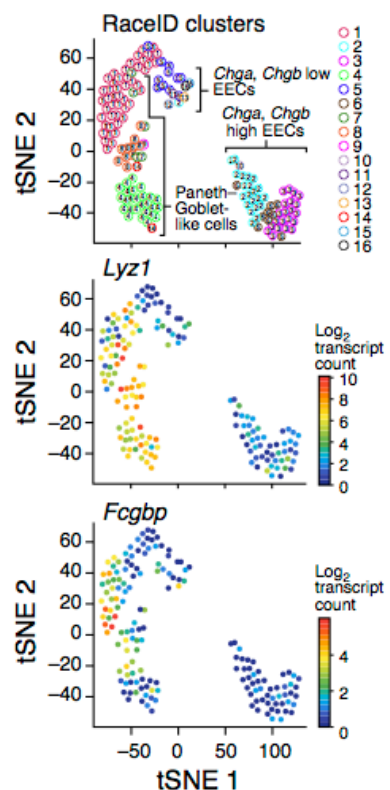
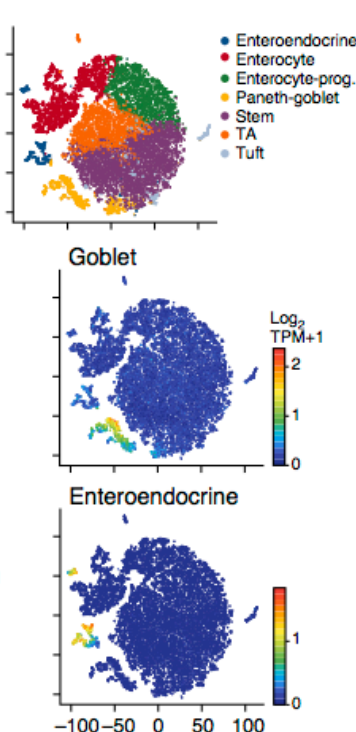

tSNE 1
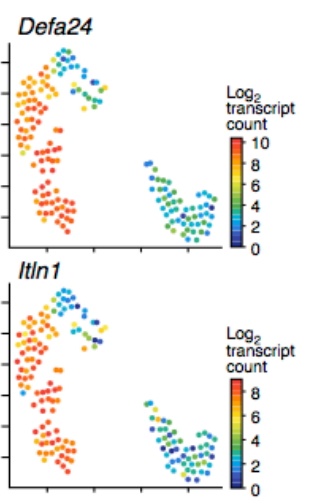

Tff3

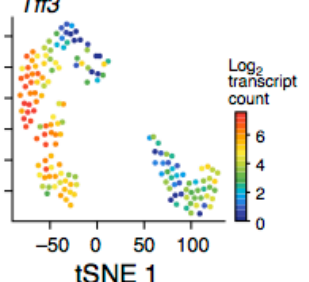

E
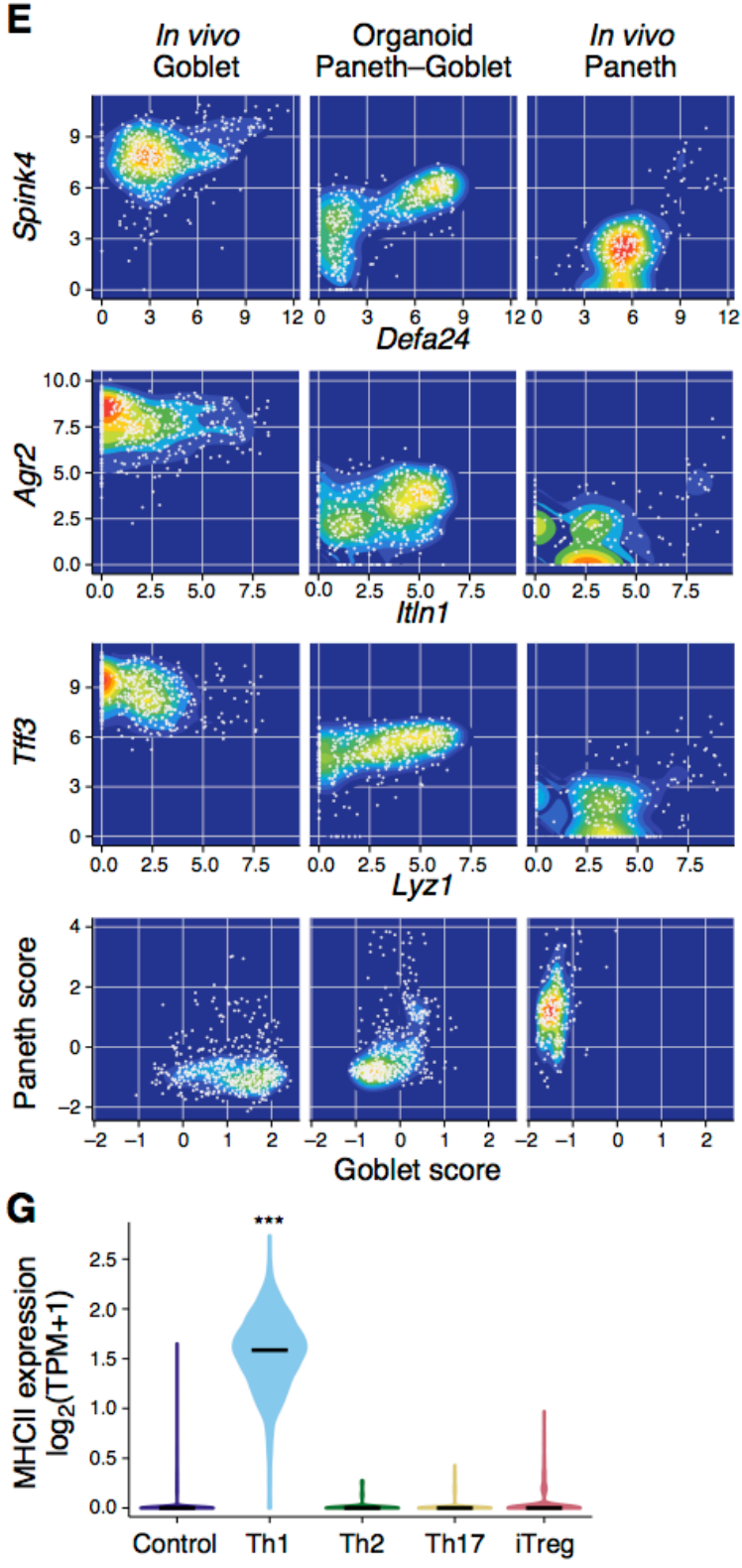


\section{Supplementary Figure 6}

A

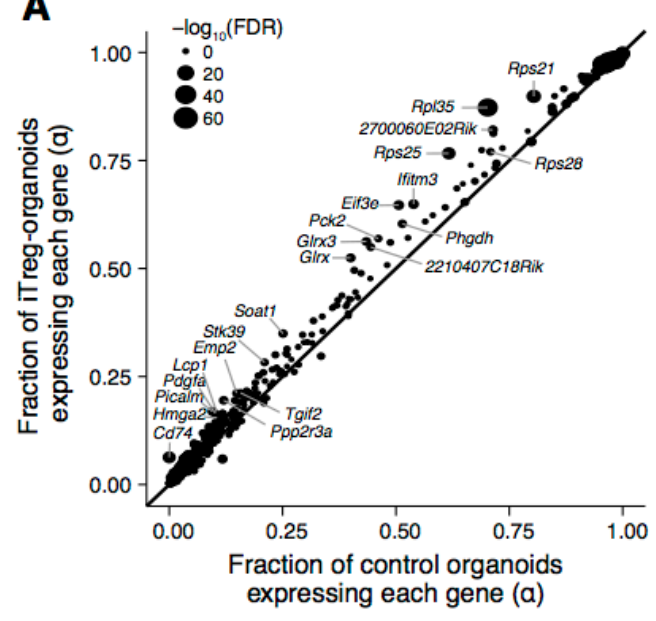

B

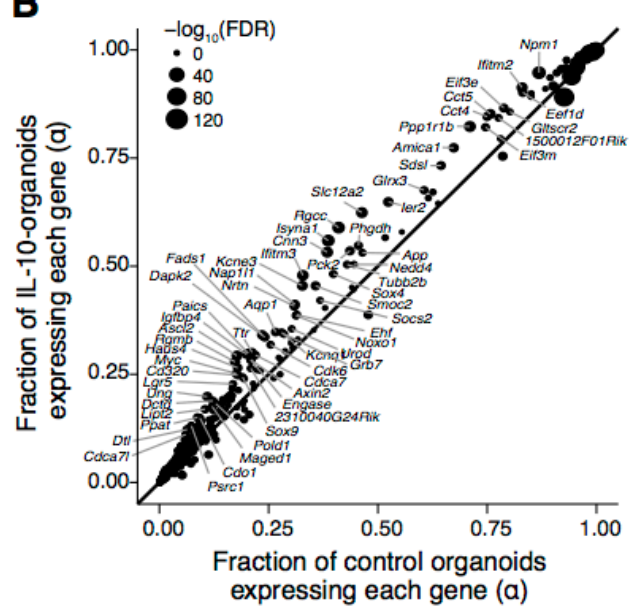

C
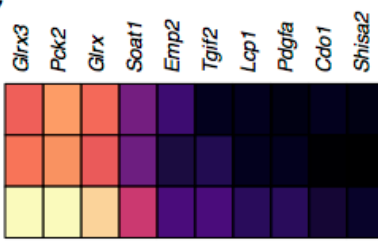
Th1 Control Treg

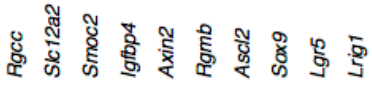

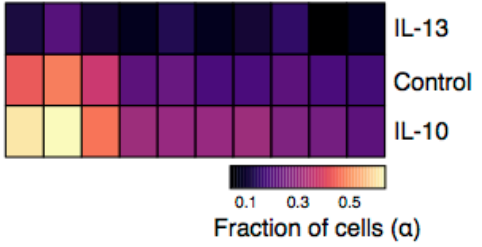

D

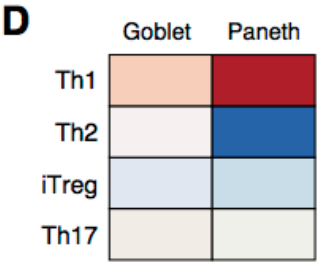

$\square_{012345}-\log _{10} p$-value (enrichment)
E

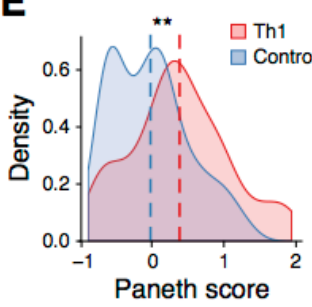

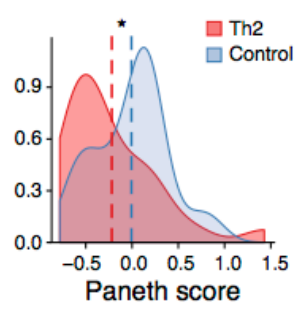

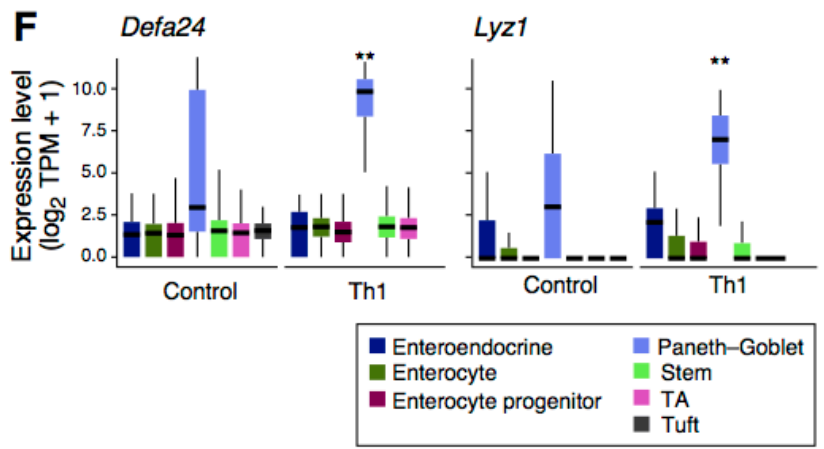




\section{Supplementary Figure 7}
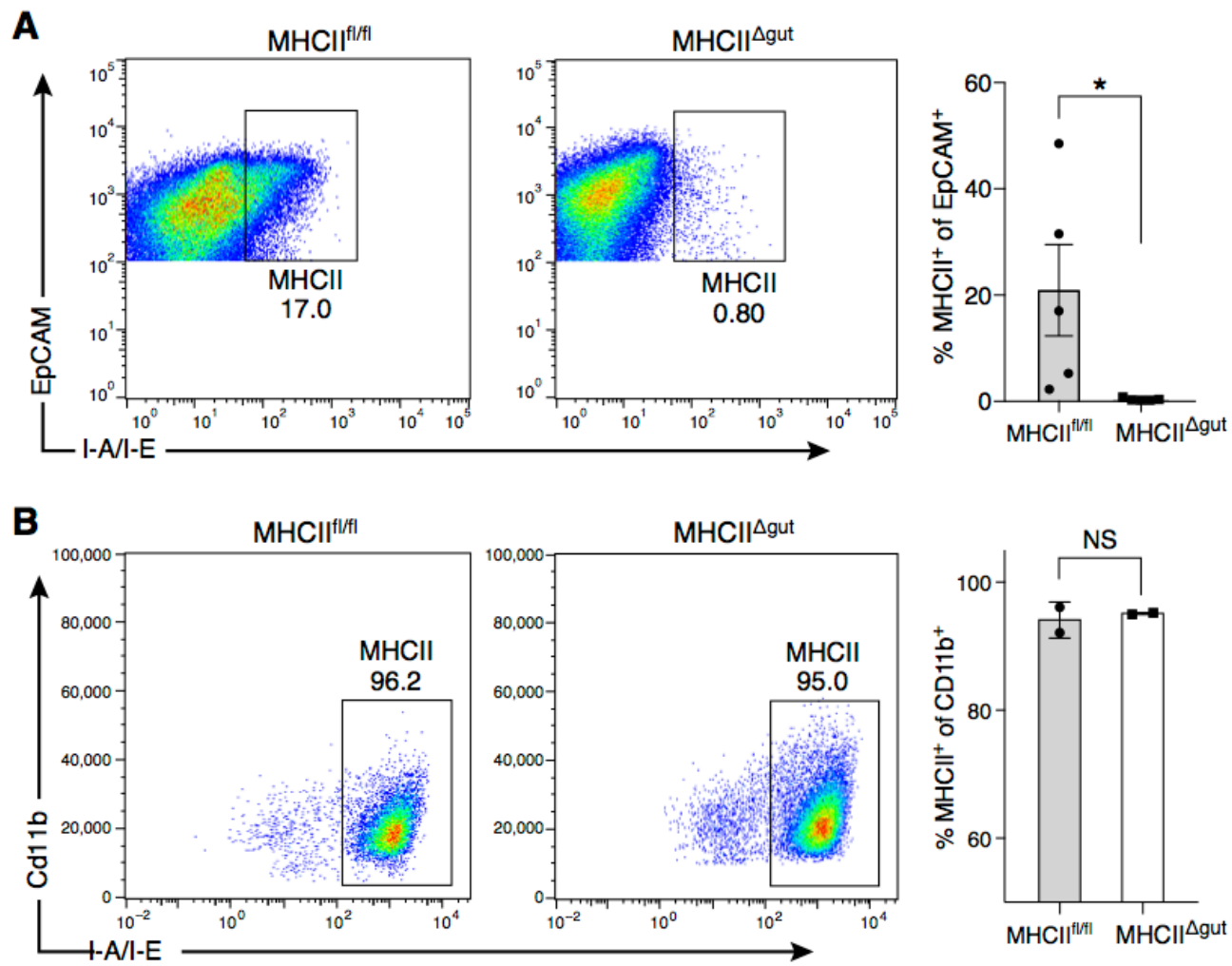


\section{Supplementary Figure 8}

A

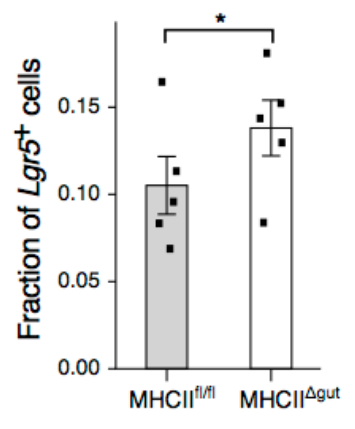

C

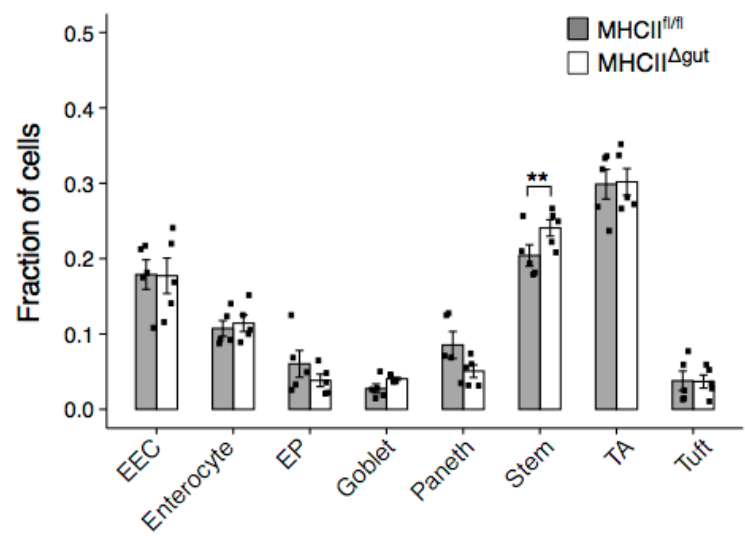

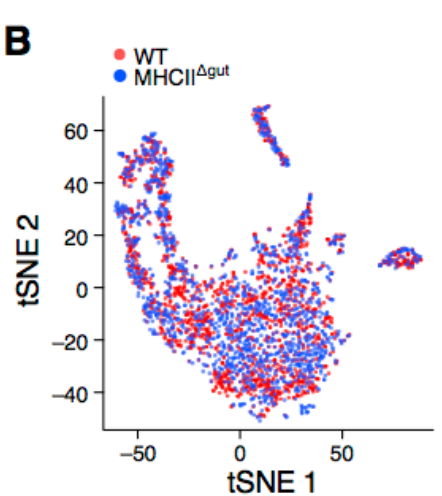

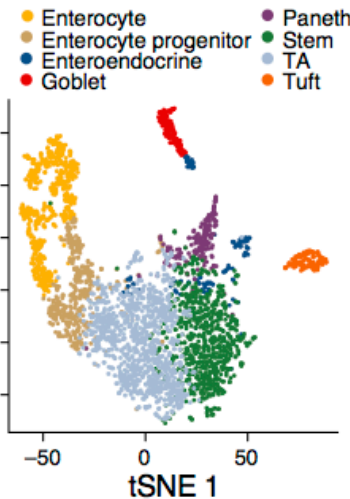

Paneth

Sem

Stemness score $\log _{2}(\mathrm{TPM}+1)$
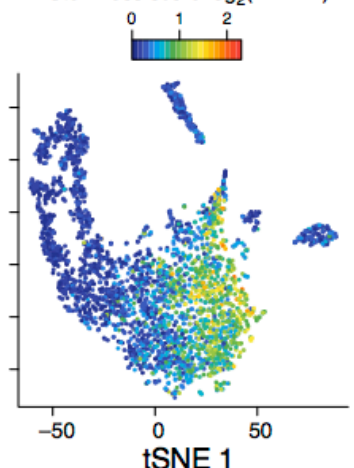

D

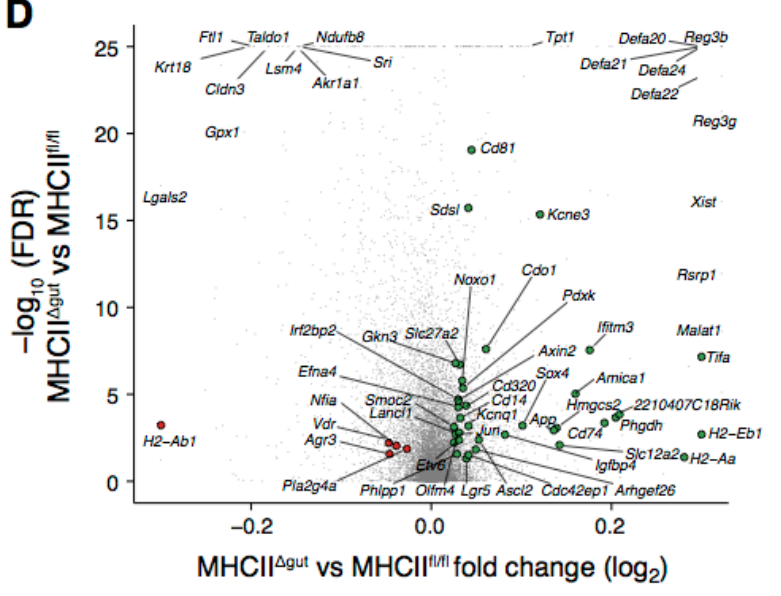

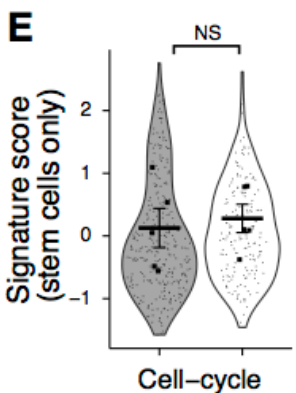
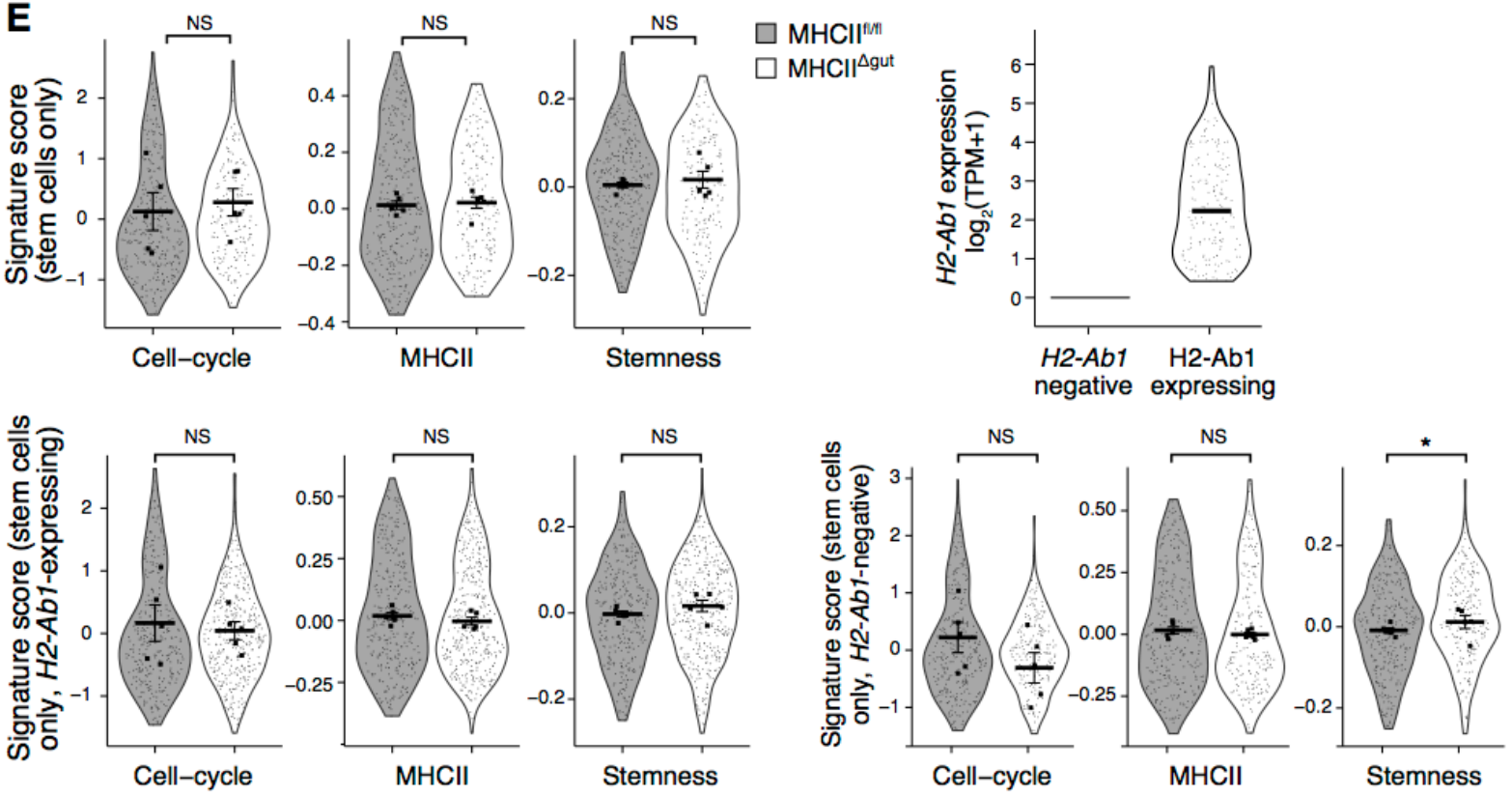


\section{Supplementary Figure 9}

A

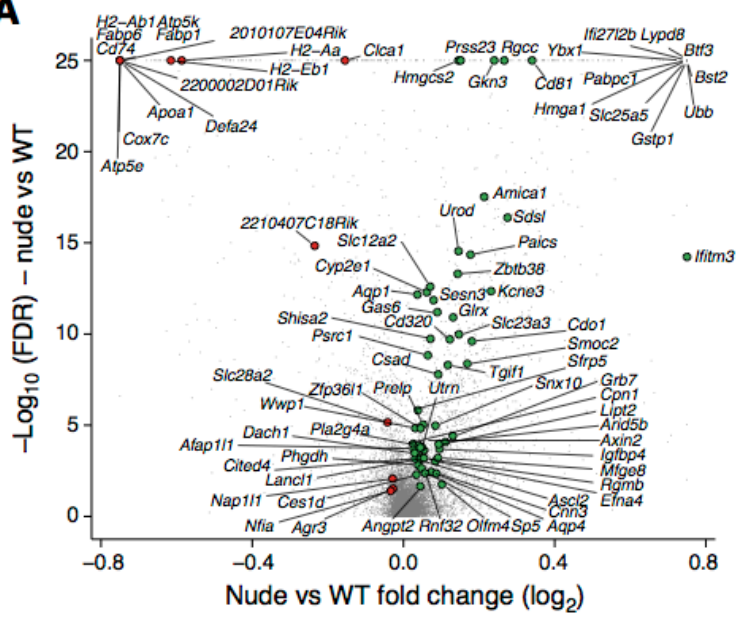

B
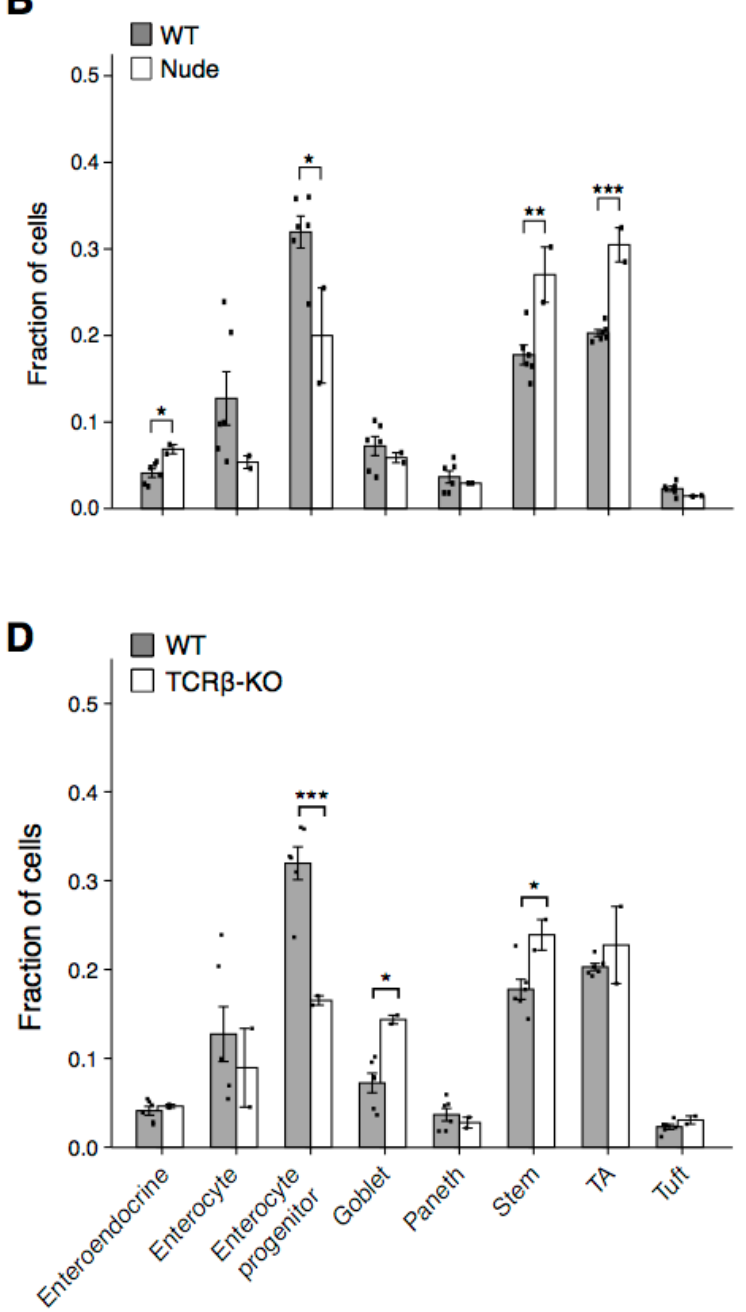

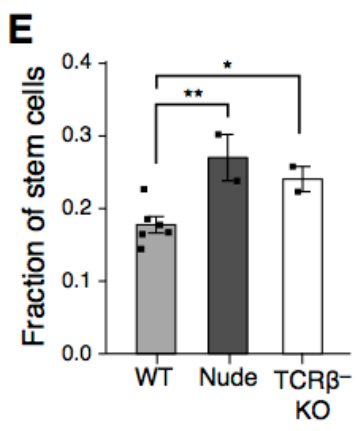

$\mathbf{F}$

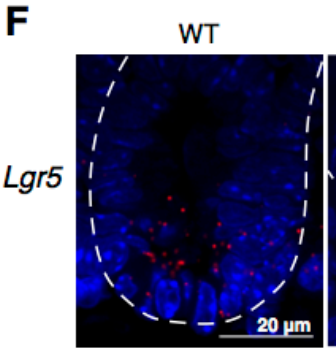

TCR $\beta$ KO

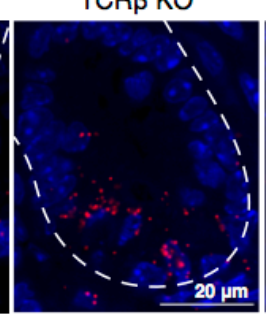

Nude

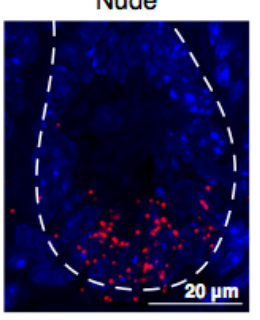

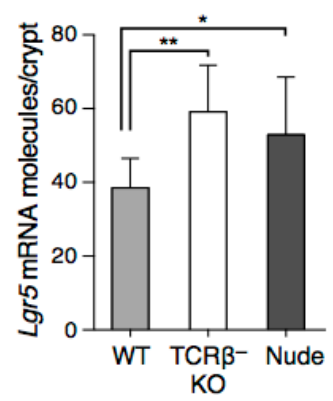




\section{Supplementary Figure 10}

A
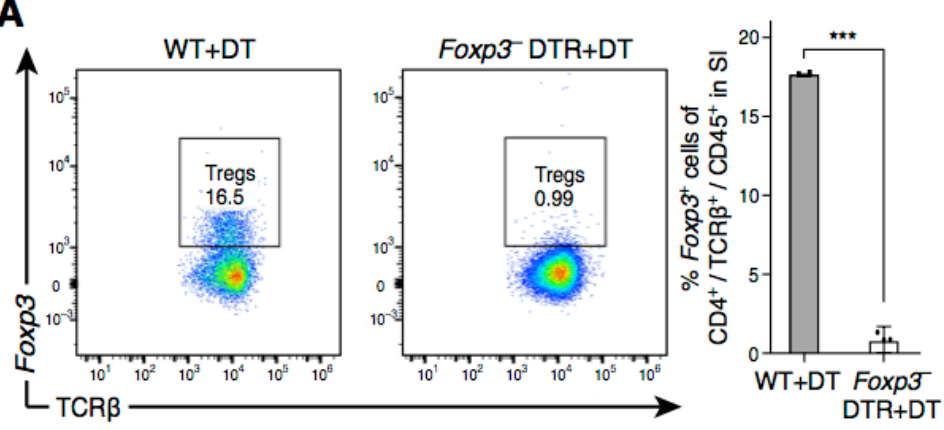

B

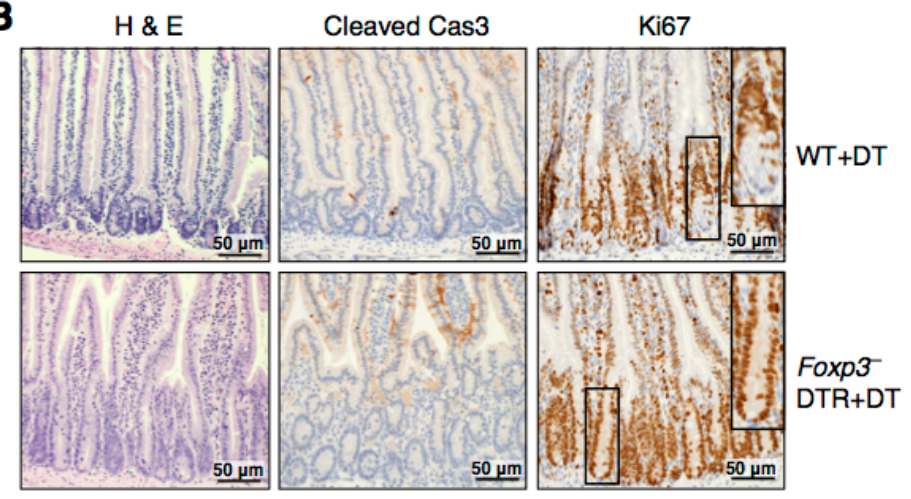

C

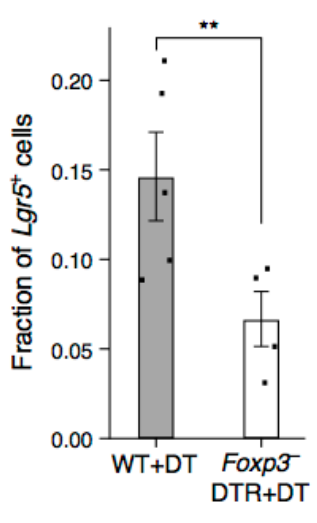

$\mathbf{F}$

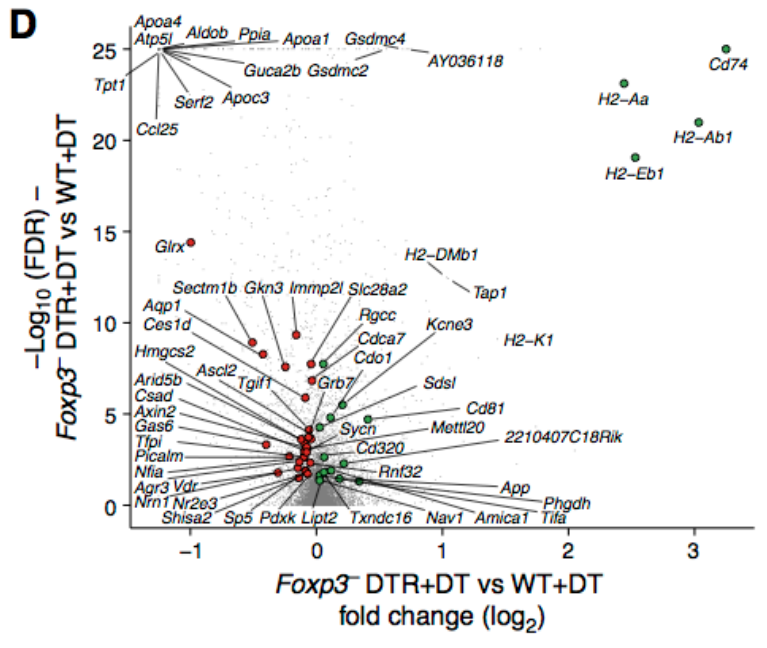

E

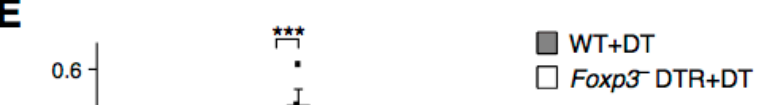

Foxp3 DTR+DT

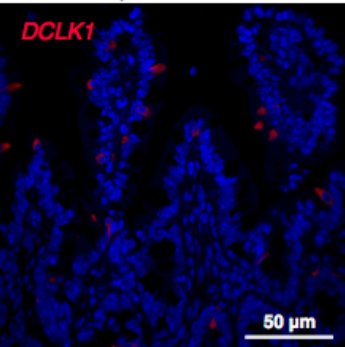

